\title{
Hamiltonian closures in fluid models for plasmas
}

\author{
E. Tassi $^{1}$ \\ 1 Aix Marseille Univ, Univ Toulon, CNRS, CPT, Marseille, France
}

\begin{abstract}
This article reviews recent activity on the Hamiltonian formulation of fluid models for plasmas in the non-dissipative limit, with emphasis on the relations between the fluid closures adopted for the different models and the Hamiltonian structures. The review focuses on results obtained during the last decade, but a few classical results are also described, in order to illustrate connections with the most recent developments. With the hope of making the review accessible not only to specialists in the field, an introduction to the mathematical tools applied in the Hamiltonian formalism for continuum models is provided. Subsequently, we review the Hamiltonian formulation of models based on the magnetohydrodynamics description, including those based on the adiabatic and double adiabatic closure. It is shown how Dirac's theory of constrained Hamiltonian systems can be applied to impose the incompressibility closure on a magnetohydrodynamic model and how an extended version of barotropic magnetohydrodynamics, accounting for two-fluid effects, is amenable to a Hamiltonian formulation. Hamiltonian reduced fluid models, valid in the presence of a strong magnetic field, are also reviewed. In particular, reduced magnetohydrodynamics and models assuming cold ions and different closures for the electron fluid are discussed. Hamiltonian models relaxing the cold-ion assumption are then introduced. These include models where finite Larmor radius effects are added by means of the gyromap technique, and gyrofluid models. Numerical simulations of Hamiltonian reduced fluid models investigating the phenomenon of magnetic reconnection are illustrated. The last part of the review concerns recent results based on the derivation of closures preserving a Hamiltonian structure, based on the Hamiltonian structure of parent kinetic models. Identification of such closures for fluid models derived from kinetic systems based on the Vlasov and drift-kinetic equations are presented, and connections with previously discussed fluid models are pointed out.
\end{abstract}




\section{INTRODUCTION}

Considerable progress in the understanding of the dynamics of liquids, gases and plasmas was obtained by making use of fluid models based on the Eulerian point of view. In such models, the behavior of liquids, gases and plasmas is described in terms of a finite set of macroscopic variables, depending on spatial coordinates and time. Various approaches, in particular, were followed, over the years, in order to derive closed fluid models for plasmas. Since the pioneering works of Chapman and Enskog in 1916 [1] and Grad [2], a considerable effort was made in order to derive fluid models for gases and plasmas, starting from kinetic equations. The latter form of models describes the dynamics of out-of-equilibrium gases and plasmas in terms of distribution functions. Supposing that the gas or plasma under consideration is composed by $\mathcal{N}$ species of particles, with $\mathcal{N}$ a positive integer, the distribution function $f_{\alpha}(\mathbf{x}, \mathbf{v}, t)$, with $\alpha=1, \cdots, \mathcal{N}$ numbering the species, is a function of spatial coordinates $\mathbf{x}$, velocity coordinates $\mathbf{v}$ and time $t$. Although in many applications the dependence on some coordinates can be ignored, in general one considers a gas or a plasma occupying a volume of space described by three coordinates $\mathbf{x}=(x, y, z)$, that we suppose to be Cartesian. The particle velocity space, on the other hand, is described by velocity coordinates $\mathbf{v}=\left(v_{x}, v_{y}, v_{z}\right)$. The physical interpretation of the distribution function $f_{\alpha}$ is then the following: the quantity $f_{\alpha}(\mathbf{x}, \mathbf{v}, t) d x d y d z d v_{x} d v_{y} d v_{z}$ corresponds to the number of particles of the species $\alpha$ contained, at the time $t$, in the volume $d x d y d z d v_{x} d v_{y} d v_{z}$ centered on the point $(\mathbf{x}, \mathbf{v})$ in a six-dimensional space; $\mathbf{x}$ indicates a point in the space occupied by the gas or the plasma, and $\mathbf{v}$ indicates the particle velocity. Kinetic models describe then the evolution of the distribution functions associated with the different particle species. As a prototypical example of kinetic model we can consider the following transport equation, that we take in the following form:

$$
\frac{\partial f_{\alpha}}{\partial t}+\mathbf{v} \cdot \nabla f_{\alpha}+\frac{\mathbf{F}_{\alpha}(\mathbf{x})}{m_{\alpha}} \cdot \frac{\partial f_{\alpha}}{\partial \mathbf{v}}=\sum_{\beta=1}^{\mathcal{N}} C_{\alpha \beta}\left(f_{\alpha}, f_{\beta}\right)
$$

where $\mathbf{F}_{\alpha}$ is a space-dependent external force acting on the $\alpha$-th particle species, $m_{\alpha}$ is the mass of the particle of species $\alpha$ and $C_{\alpha \beta}$ are operators, that we leave unspecified, 
that account for the collisions between particles of species $\alpha$ and $\beta$. The second term in Eq. (1), on the other hand, accounts for temporal variations of the distribution function due to the free flow of particles. The derivation of fluid models from a kinetic model, as described in ordinary textbooks on gas dynamics and plasma physics, proceeds with the introduction of the moments of the distribution functions. In the case of distribution functions $f_{\alpha}(\mathbf{x}, \mathbf{v}, t)$ defined as above, given a non-negative integer $S$, a moment of order $S$ is a weighted average of a polynomial $B_{\alpha}(\mathbf{v})$ of degree $S$ in velocity space, i.e. a quantity of the form $\int d^{3} v B_{\alpha}(\mathbf{v}) f_{\alpha}(\mathbf{x}, \mathbf{v}, t)$, where

$$
B_{\alpha}(\mathbf{v})=\sum_{i_{1}=0}^{s_{1}} \sum_{i_{2}=0}^{s_{2}} \sum_{i_{3}=0}^{s_{3}} a_{i_{1} i_{2} i_{3}}^{\alpha} v_{x}^{i_{1}} v_{y}^{i_{2}} v_{z}^{i_{3}}
$$

with $a_{i_{1} i_{2} i_{3}}^{\alpha}$ constant coefficients and $s_{1}, s_{2}$ and $s_{3}$ three non-negative integers such that $s_{1}+s_{2}+s_{3}=S$.

Moments are related to macroscopic fluid variables. In particular, the zeroth order moment corresponds to the particle density $n_{\alpha}$ of the species $\alpha$, i.e.

$$
n_{\alpha}(\mathbf{x}, t)=\int d^{3} v f_{\alpha}(\mathbf{x}, \mathbf{v}, t)
$$

The three components $v_{\alpha x}, v_{\alpha y}, v_{\alpha z}$ of the velocity vector field $\mathbf{v}_{\alpha}(\mathbf{x}, t)$, in the Eulerian point of view, of the fluid associated with the species $\alpha$ are related to the first order moments by

$$
n_{\alpha}(\mathbf{x}, t) v_{\alpha i}(\mathbf{x}, t)=\int d^{3} v v_{i} f_{\alpha}(\mathbf{x}, \mathbf{v}, t), \quad i=x, y, z
$$

The scalar pressure $p_{\alpha}(\mathbf{x}, t)$ of the particles of species $\alpha$, on the other hand, involves second order moments according to the definition

$$
p_{\alpha}(\mathbf{x}, t)=\frac{1}{3} m_{\alpha} \int d^{3} v\left|\mathbf{v}-\mathbf{v}_{\alpha}(\mathbf{x}, t)\right|^{2} f_{\alpha}(\mathbf{x}, \mathbf{v}, t)
$$

The components $q_{\alpha x}, q_{\alpha y}, q_{\alpha z}$ of the heat flux (density) vector field $\mathbf{q}_{\alpha}(\mathbf{x}, t)$, finally, are related to third order moments by

$$
q_{\alpha i}(\mathbf{x}, t)=\frac{1}{2} m_{\alpha} \int d^{3} v\left(v_{i}-v_{\alpha i}(\mathbf{x}, t)\right)\left|\mathbf{v}-\mathbf{v}_{\alpha}(\mathbf{x}, t)\right|^{2} f_{\alpha}(\mathbf{x}, \mathbf{v}, t), \quad i=x, y, z
$$


The fields $n_{\alpha}, \mathbf{v}_{\alpha}, p_{\alpha}$ and $\mathbf{q}_{\alpha}$ represent customary macroscopic variables in terms of which fluid models are formulated. We remark that the definitions (3), (4), (5) and (6) are those commonly adopted for plasma variables, which might differ from those adopted in the case of mixtures of neutral gases [1].

From the definitions (3), (4), (5) and (6) it follows that such macroscopic variables can be expressed in terms of the particular choice of moments

$$
P_{s_{1} s_{2} s_{3}}(\mathbf{x}, t)=\int d^{3} v v_{x}^{s_{1}} v_{y}^{s_{2}} v_{z}^{s_{3}} f_{\alpha}(\mathbf{x}, \mathbf{v}, t)
$$

Evolution equations for the moments $P_{s_{1} s_{2} s_{3}}$ (and, consequently, for the fluid variables) can be obtained from the kinetic equations. We consider, as extremely simplified example, the case of the transport equation (1) in the absence of forces and collisions, i.e. for $\mathbf{F}_{\alpha}=C_{\alpha \beta}=$ 0. From Eq. (1), after multiplying each term of the equation times $v_{x}^{s_{1}} v_{y}^{s_{2}} v_{z}^{s_{3}}$ and integrating over velocity space, one then immediately obtains

$$
\frac{\partial P_{s_{1} s_{2} s_{3}}}{\partial t}+\frac{\partial P_{s_{1}+1 s_{2} s_{3}}}{\partial x}+\frac{\partial P_{s_{1} s_{2}+1 s_{3}}}{\partial y}+\frac{\partial P_{s_{1} s_{2} s_{3}+1}}{\partial z}=0
$$

It follows from Eq. (8) that the evolution of a moment of order $S$ requires the knowledge of moments of order $S+1$. Equation (8) leads then to an infinite hierarchy of evolution equations. Obtaining a closed fluid model evolving a finite number of macroscopic variables, requires then reducing the above infinite hierarchy to a finite set of equations. This can be achieved by assuming a closure relation. Fixing an order of moment $S$, supposed to account for the number of fluid variables that one considers appropriate for the phenomenon to describe, assuming a closure relation amounts to imposing a relation between moments of order $S+1$ and lower order moments. For instance, it turns out that, in order to derive a fluid model adopting as dynamical variables only $n_{\alpha}, \mathbf{v}_{\alpha}$ and $p_{\alpha}$, one can assume a closure relation $\mathbf{q}_{\alpha}=\mathbf{Q}\left(n_{\alpha}, \mathbf{v}_{\alpha}, p_{\alpha}\right)$, where $\mathbf{Q}$ is, in general, an operator, acting on the lower-order moments. In this way, evolution equations for the moments of order higher than two will no longer be required to determine the evolution of $n_{\alpha}, \mathbf{v}_{\alpha}$ and $p_{\alpha}$. Although extremely simplifed, the example of Eq. (8) is sufficient to introduce the closure problem, which manifests itself 
already with the sole presence of the free flow term in the parent kinetic equation (1). Clearly, the problem becomes of interest when physically more realistic kinetic models are adopted as parent models for deriving fluid models. In particular, in the case of plasmas, which is the case of interest in the present article, the forces $\mathbf{F}_{\alpha}$ acting on particle populations, often cannot be assumed to be external but they depend on electromagnetic fields, whose evolution is in turn affected by the motion of the charged particles composing the plasma. Parent kinetic equations are then typically coupled with Maxwell's equations governing the electromagnetic fields.

Evidently, in deriving a fluid model for plasmas through a closure procedure, a crucial question concerns how to determine the closure relation. One straightforward possibility is to postulate an equation of state motivated by the knowledge of some features of the physical process under consideration. For instance, if the characteristic time scale of the process involved is much shorter than the characteristic time of heat transfer, heat flux can be neglected and an adiabatic equation of state relating the pressure with the density and the entropy could be adopted. Although convenient for some applications, such an approach can become insufficient, for instance, if details on heat transport are important for the fluid model. More rigorous approaches based on systematic derivations of higher-order moment closures were followed, leading to remarkable results. Closed set of fluid equations in collisional plasmas close to equilibrium were derived following an asymptotic procedure in the classical works of Refs. [3, 4]. We indicate Ref. [5] for further references about the considerable amount of results on the derivation of fluid equations for classical transport in plasmas.

Another criterion adopted to determine advanced closure relations consists of incorporating kinetic effects in the fluid model, in particular at the level of the linear theory. This led to the development of so called Landau-fluid models [6-11]. In these models, the closure is designed in such a way that the linear dispersion relation approximates that of the parent kinetic model, and in particular it accounts for a kinetic, wave-particle effect, such as Landau damping. A fluid closure including wave-particle effects also in the nonlinear regime 
was proposed in Ref. [12, 13]. Non-dissipative closures for the linearly unstable modes of the heat flux were presented in Ref. [14] and were shown to be able to reproduce nonlinear behavior of the parent kinetic model undergoing an ion temperature gradient instability.

The emergence of gyrokinetic models (see, e.g. Ref. [15]), initially motivated by the investigation of low-frequency plasma turbulence in tokamaks, led to plasma descriptions based on distribution functions defined over a reduced set of coordinates, i.e., fewer than the six coordinates considered, for instance, in the example of Eq. (1). Fluid models were then derived also adopting the gyrokinetic equations as parent kinetic model and leading to the so-called gyrofluid models [16]. Because of the presence, in the gyrokinetic equations, of operators accounting for the average of fields over the orbit of a charged particle around the magnetic field, closure problems for gyrofluid models possess the intricacy of involving all moments of the distribution function with respect to the magnetic moment coordinate. A number of closed gyrofluid models [17-25] were proposed, most of them including also Landau-fluid effects. We remark that the gyrofluid closures in Ref. [24] were designed in order to guarantee energy conservation in the absence of dissipative terms.

A further customary approach in deriving fluid models for plasmas, consists of taking already closed fluid models as parent models and then performing operations on these, in order to obtain simpler fluid models, specifically adapted to describe the phenomenon of interest. Such operations can be, for instance, the asymptotic expansion of the equations of motion of the parent model in terms of a small parameter, as is done for deriving the low- $\beta$ reduced magnetohydrodynamics (MHD) equations [26]. The drift approximation (see. e.g. Ref. [27]), valid for phenomena with characteristic frequency much lower than the ion cyclotron frequency, is another common procedure followed to derive reduced fluid models. Classical examples of such models include the Hasegawa-Mima [28] and the Hasegawa-Wakatani equations [29], as well as several other models discussed in this review such as those of Refs. [6, 30-32]. Also, simplified models can be derived by truncating a parent fluid model with a lower-order closure, based on a phenomenological argument (for instance, imposing an isothermal closure into a model evolving temperature fluctuations). With such a procedure 
one can derive, for instance, the models described in Refs. [33-35]. MHD models can also be derived using multiple-fluid systems as parent models. For MHD models, the closures can be analogous to those adopted for ordinary fluids (as in the barotropic and incompressible case), or influenced by the presence of the magnetic field, as is the case of the double adiabatic MHD closure [36].

Certainly, the derivation of fluid models for plasma has a long history and an enormous literature is available on the subject. Nevertheless, in spite of the considerable progress made in this field, some aspects still received relatively little attention and leave open questions. One such aspect concerns the impact that closure relations can have on some structure that the parent model might possess, such as for instance a Hamiltonian structure.

Indeed, ultimately, the behavior of classical plasmas consists of the dynamics of charged particles interacting with electromagnetic fields. Such a system possesses a Hamiltonian structure descending from an action principle [37]. In practice, currently adopted models are derived from parent (kinetic or fluid) models which in general do not have a Hamiltonian structure and contain dissipative terms, such as for instance viscosity in fluid models or collisional terms in kinetic models. When such terms are omitted from the parent model, the resulting model is supposed to possess a Hamiltonian structure, reminiscent of the fundamental Hamiltonian character of the underlying microscopic particle dynamics. The dissipationless limit of the parent model would then possess a total conserved energy, and, in general, a noncanonical Hamiltonian structure, which is the typical situation for continuum models in Eulerian variables (see, e.g. Refs. [38, 39] as well as Sec. II). For noncanonical Hamiltonian systems, in addition to energy conservation, the associated dynamics will be constrained also by the conservation of Casimir invariants. It is then desirable that also in the derived fluid model it is possible to clearly identify dissipative terms, in the absence of which, the model possesses a Hamiltonian structure. Dissipative terms could then of course be reintroduced a posteriori to complete the modelling. It is however, often the case that the impact of the closure on the structure of the derived fluid model, whether this is derived directly by closing a kinetic system or from operations performed on a parent fluid model, 
is not known. In particular, it is often not clear whether the derived fluid model possesses a Hamiltonian structure, once the terms that, supposedly, lead to dissipation, have been removed. We point out that the knowledge of the Hamiltonian structure of a fluid model in the non-dissipative limit is not only important from a fundamental point of view. An unconscious non-Hamiltonian character of the model in its non-dissipative limit might, for instance, lead to physically questionable instabilities [40]. The knowledge of the Hamiltonian structure, on the other hand, leads to an unambiguous identification of the energy of the system, an essential information for investigating, for instance, energy transfers in turbulent plasmas. In addition to the knowledge of the energy, the knowledge of the Casimir invariants can provide further conserved quantities to be used for checking the quality of numerical simulations. A further application of the Hamiltonian structure is related to stability analysis, in particular by means of the Energy-Casimir method (see, e.g. Refs. [38, 41] as well as Refs. [42-45] and references therein), which applies namely to noncanonical Hamiltonian systems. The noncanonical Hamiltonian approach is also relevant for the equilibrium statistical mechanics approach to two-dimensional turbulent flows [46]. We mention also a recent application of the Hamiltonian formalism for the derivation of nonlinear waves and a subsequent application for the analysis of turbulence in the solar wind [47, 48]. These arguments motivate an effort toward the identification of Hamiltonian structures in fluid models for plasmas. This subject has now a history lasting almost four decades and had a fundamental breakthrough in 1980 with the discovery of the noncanonical Hamiltonian structure of the ideal adiabatic MHD equations [49]. This result was followed by a series of works concerning the derivation of Hamiltonian structures for various fluid models for plasmas, that will be cited and, to some extent, described in this article. These concerned refined versions of MHD theories, reduced models for strongly magnetized plasmas, models accounting for finite-Larmor-radius (FLR) effects and gyrofluid models. Different fluid closure schemes have been shown to be compatible with a Hamiltonian structure in the non-dissipative limit.

In spite of the progress made on the subject during the last decades, however, still a num- 
ber of important problems are open and systematic ways for deriving closures guaranteeing a Hamiltonian structure are still under development.

The purpose of this article is to review the results obtained in the identification and/or derivation of Hamiltonian structures of fluid models for plasmas in the absence of dissipation. We will consider, on one hand, models derived from parent fluid models assuming a certain closure relation and for which the Hamiltonian structure was found a posteriori, or obtained by construction. On the other hand, we will also present results concerning the derivation of closures, from parent kinetic models, which guarantee a Hamiltonian structure in the resulting fluid model.

The review focuses essentially on the results obtained during the last ten years. Nevertheless, some older results, such as for instance the Hamiltonian formulation of adiabatic MHD, reduced MHD or of the four-field model with magnetic curvature, are also described, as we thought they could be propaedeutical to and show interesting connections with the more recent results.

Given that the development of the subject of research treated in this article was, so far, essentially on the theoretical side, the review presents theoretical, and, mostly analytical results. A clear deficiency of this article is then the lack of contact with experimental results. In this respect, a few perspectives are mentioned at the end of Sec. VI.

In the hope of making this review self-contained and accessible to a reader possibly unfamiliar with the subject, the article begins in Sec. II with a short introduction to the Hamiltonian formalism for continuum models for plasmas. Basic mathematical tools subsequently used in the article are recalled and illustrated with the example of the model for a barotropic fluid. Section III deals with Hamiltonian MHD models. Starting with the classical result on adiabatic MHD, it is then shown how the double-adiabatic closure also admits a Hamiltonian formulation and how one can impose the incompressibility closure, while preserving a Hamiltonian structure, by means of Dirac's theory of constraints. The Section concludes illustrating the Hamiltonian structure of an extended version of MHD, accounting for two-fluid effects, with a barotropic closure. Section IV reviews results on 
reduced fluid models, which apply to weakly nonlinear plasmas in the presence of a magnetic field with an intense component along one direction. Various reduced drift-fluid models, valid on scales larger than the ion Larmor radius and characterized by different closures, are presented and their Hamiltonian structures are discussed and compared. Subsequently, it is shown how the gyromap technique can help in adding ion FLR effects to a Hamiltonian cold-ion model, while preserving a Hamiltonian structure. The final part of the Section is devoted to gyrofluid models and illustrates, in particular, a recent five-field and a six-field Hamiltonian gyrofluid models. Connections with previous Hamiltonian gyrofluid models characterized by other closures are discussed. Sections III and IV concern Hamiltonian fluid models derived from parent fluid models, in most cases postulating a closure relation and reconstructing the Hamiltonian structure a posteriori, or, as is the case for the incompressible closure for MHD with Dirac's constraints and the gyromap for reduced models, obtaining the Hamiltonian structure by construction. In Sec. V, on the other hand, we present recent results concerning strategies to derive, from the Hamiltonian structure of parent kinetic models, closures yielding a Hamiltonian structure in the resulting fluid model. Applications to models derived from Vlasov and drift-kinetic systems are illustrated and connections with previously discussed models are shown. Section VI is devoted to conclusions and perspectives. An Appendix illustrating the proof of the Jacobi identity for the Poisson bracket of the barotropic fluid model complements the review.

\section{HAMILTONIAN DESCRIPTION OF CONTINUUM MODELS FOR PLAS- MAS : TECHNICAL PRELIMINARIES}

The purpose of this Section is to provide a concise introduction to the basic concepts and tools in the Hamiltonian formulation of continuum models for plasmas, the use of which will be frequently made in this article. This Section is meant just to provide a practical introduction limited to the class of models described in the article. Consequently, it is by no means complete, and emphasis is more on a working knowledge than on rigor. More general, rigorous and detailed expositions of the subject can be found, for instance, in Refs. 
$[38,39,50]$. This Section can of course be skipped by the reader already familiar with the subject.

\section{A. Hamiltonian basics}

The fluid and kinetic models treated in this article will be systems of partial differential equations of the form

$$
\frac{\partial \chi_{i}}{\partial t}=F_{i}\left(\chi_{1}, \cdots, \chi_{N}\right), \quad i=1, \cdots, N
$$

In Eq. (9), $\chi_{1}, \cdots, \chi_{N}$ (where $N$ is a non-negative integer) form a set of field variables $\chi_{i}\left(\mathbf{z}_{(i)}, t\right)$, with $i=1, \cdots, N$, depending on time $t$ and on coordinates describing points $\mathbf{z}_{(i)} \in \mathcal{D}_{(i)} \subseteq \mathbb{R}^{n_{(i)}}$. On the right-hand side of Eq. (9), $F_{1}, \cdots, F_{N}$ indicate, in general, nonlinear integro/differential operators, acting on the field variables. In this context, typical examples of field variables will be the components of the electromagnetic fields, the distribution functions appearing in kinetic models, or the macroscopic fluid variables, such as, for instance, density or pressure, involved in fluid models. Because the same model can involve fields depending on different sets of coordinates (typically the distribution functions depending on velocity and spatial coordinates and the fluid variables, as well as the electromagnetic field, depending on the spatial coordinates only), the index $(i)$ was added to the set of coordinates. We will assume that the first $M$ fields of each model (with $0 \leq M \leq N$ ) depend on spatial and velocity coordinates, denoted as $\mathbf{z}_{\mathbf{x}, \mathbf{v}}$, and that the remaining $N-M$ fields depend only on the spatial coordinates, denoted as $\mathbf{z}_{\mathbf{x}}$. We can then write $\mathbf{z}_{(1)}=$ $\mathbf{z}_{(2)}=\cdots=\mathbf{z}_{(M)} \equiv \mathbf{z}_{\mathbf{x}, \mathbf{v}}$ and $\mathbf{z}_{(M+1)}=\mathbf{z}_{(M+2)}=\cdots=\mathbf{z}_{(N)} \equiv \mathbf{z}_{\mathbf{x}}$. Consequently, we also have $\mathcal{D}_{(1)}=\mathcal{D}_{(2)}=\cdots=\mathcal{D}_{(M)} \equiv \mathcal{D}_{\mathbf{x}, \mathbf{v}} \subseteq \mathbb{R}^{m+n}$ and $\mathcal{D}_{(M+1)}=\mathcal{D}_{(M+2)}=\cdots=\mathcal{D}_{(N)} \equiv \mathcal{D}_{\mathbf{x}} \subseteq \mathbb{R}^{n}$, where $m$ and $n$ are non-negative integers and where we indicated with $\mathcal{D}_{\mathbf{x}, \mathbf{v}}$ the domain of the spatial and velocity coordinates and with $\mathcal{D}_{\mathbf{x}}$ the domain involving the spatial coordinates only. Note that $\mathcal{D}_{\mathbf{x}, \mathbf{v}}$ will always be of the form $\mathcal{D}_{\mathbf{x}, \mathbf{v}}=\mathcal{D}_{\mathbf{x}} \times \mathcal{D}_{\mathbf{v}}$, where $\mathcal{D}_{\mathbf{v}}$ is the domain of the velocity coordinates. The results presented in this review, can be formulated with $\mathcal{D}_{\mathbf{x}} \subset \mathbb{R}^{n}$ a compact, boundary-less (periodic) domain. Specific choices of the domain 
will be indicated in some cases, though. We will also set $\mathcal{D}_{\mathbf{v}}=\mathbb{R}^{m}$. For instance, the one-dimensional Vlasov-Ampère system, which will be treated later, evolves two fields: the distribution function $\chi_{1}=f(x, v, t)$, for which $\mathbf{z}_{(1)}=\mathbf{z}_{\mathbf{x}, \mathbf{v}}=(x, v) \in[0,1] \times \mathbb{R} \equiv \mathcal{D}_{\mathbf{x}, \mathbf{v}}$, and the electric field $\chi_{2}=E(x, t)$, for which $\mathbf{z}_{(2)}=\mathbf{z}_{\mathbf{x}}=x \in[0,1] \equiv \mathcal{D}_{\mathbf{x}}$. In most of the cases treated in this article, however, the set of coordinates will be the same for every field of a given model.

At this point it is convenient, for a given system of the form (9), to denote with $P$ the space of functions in which the field variables belong. An element $\chi \in P$ will then consist of an $N$-tuple $\chi=\left(\chi_{1}, \cdots, \chi_{N}\right)$. We will denote by $\mathcal{F}(P)$ the set of functionals $F: P \rightarrow \mathbb{R}$.

We say that a system of the form (9), defined over a space of field variables $P$ is Hamiltonian, if there exists a Poisson bracket $\{\}:, \mathcal{F}(P) \times \mathcal{F}(P) \rightarrow \mathcal{F}(P)$ and a Hamiltonian functional $H \in \mathcal{F}(P)$ such that the system (9) can be cast in the form

$$
\frac{\partial \chi_{i}}{\partial t}=\left\{\chi_{i}, H\right\}, \quad i=1, \cdots, N
$$

We recall that a Poisson bracket is an operator satisfying the four following properties:

(i) bilinearity: $\{F, G+H\}=\{F, G\}+\{F, H\}, \quad\{F+G, H\}=\{F, H\}+\{G, H\}$,

$$
\{\lambda F, G\}=\{F, \lambda G\}=\lambda\{F, G\},
$$

(ii) antisymmetry: $\{F, G\}=-\{G, F\}$,

(iii) Leibniz identity: $\{F G, H\}=F\{G, H\}+\{F, H\} G$,

(iv) Jacobi identity: $\{F,\{G, H\}\}+\{G,\{H, F\}\}+\{H,\{F, G\}\}=0$,

where $\lambda$ is a constant and $F, G$ and $H$ are generic elements of $\mathcal{F}(P)$.

Because a Poisson bracket acts on two functionals of $\chi \in P$ and yields again a functional of $\chi \in P$, an immediate consequence of Eq. (10), is that it must be possible to express the field variables $\chi_{1}, \cdots, \chi_{N}$ themselves, as functionals over $P$. This is accomplished by 
writing

$$
\begin{array}{r}
\chi_{i}\left(\mathbf{z}_{\mathbf{x}, \mathbf{v}}, t\right)=\int_{\mathcal{D}_{\mathbf{x}, \mathbf{v}}} d^{m+n} z_{\mathbf{x}, \mathbf{v}}^{\prime} \delta\left(\mathbf{z}_{\mathbf{x}, \mathbf{v}}^{\prime}-\mathbf{z}_{\mathbf{x}, \mathbf{v}}\right) \chi_{i}\left(\mathbf{z}_{\mathbf{x}, \mathbf{v}}^{\prime}, t\right), \quad i=1, \cdots, M \\
\chi_{i}\left(\mathbf{z}_{\mathbf{x}}, t\right)=\int_{\mathcal{D}_{\mathbf{x}}} d^{n} z_{\mathbf{x}}^{\prime} \delta\left(\mathbf{z}_{\mathbf{x}}^{\prime}-\mathbf{z}_{\mathbf{x}}\right) \chi_{i}\left(\mathbf{z}_{\mathbf{x}}^{\prime}, t\right), \quad i=M+1, \cdots, N
\end{array}
$$

where $\delta\left(\mathbf{z}_{(i)}^{\prime}-\mathbf{z}_{(i)}\right)$, for $i=1, \cdots, N$, is a Dirac delta.

More generally, the functionals of the field variables, such as the Hamiltonian, that will be involved in our context, can be written in the form

$$
\begin{aligned}
& F\left(\chi_{1}, \cdots, \chi_{N}\right) \\
& =\int_{\mathcal{D}_{\mathbf{x}, \mathbf{v}}} d^{m+n} z_{\mathbf{x}, \mathbf{v}} \mathfrak{f}_{\mathbf{x}, \mathbf{v}}\left(\mathbf{z}_{\mathbf{x}, \mathbf{v}}, \chi_{1}, \cdots, \chi_{N}, \partial_{z_{\mathbf{x}, \mathbf{v}}} \chi_{1}, \cdots, \partial_{z_{\mathbf{x}}} \chi_{N}, \partial_{z_{\mathbf{x}, \mathbf{v}} z_{\mathbf{x}, \mathbf{v}}} \chi_{1}, \cdots, \partial_{z_{\mathbf{x}} z_{\mathbf{x}}} \chi_{N}, \cdots\right) \\
& +\int_{\mathcal{D}_{\mathbf{x}}} d^{n} z_{\mathbf{x}} \mathfrak{f}_{\mathbf{x}}\left(\mathbf{z}_{\mathbf{x}}, \chi_{M+1}, \cdots, \chi_{N}, \partial_{z_{\mathbf{x}}} \chi_{M+1}, \cdots, \partial_{z_{\mathbf{x}}} \chi_{N}, \partial_{z_{\mathbf{x}} z_{\mathbf{x}}} \chi_{M+1}, \cdots, \partial_{z_{\mathbf{x}} z_{\mathbf{x}}} \chi_{N}, \cdots\right)
\end{aligned}
$$

where $\mathfrak{f}_{\mathbf{x}, \mathbf{v}}$ and $\mathfrak{f}_{\mathbf{x}}$ are operators acting on the field variables, on their derivatives of any order and on the coordinates.

On the other hand, the Poisson brackets for Hamiltonian continuum models for plasmas typically possess the form

$$
\{F, G\}=\left\langle\frac{\delta F}{\delta \chi} \mid J \frac{\delta G}{\delta \chi}\right\rangle
$$

where $J$ is a matrix operator referred to as Poisson operator and where we introduced the inner product (corresponding, in general, to the pairing between a vector space and its dual)

$$
<f \mid g>=\sum_{i=1}^{M} \int_{\mathcal{D}_{\mathbf{x}, \mathbf{v}}} d^{m+n} z_{\mathbf{x}, \mathbf{v}} f_{i} g_{i}+\sum_{i=M+1}^{N} \int_{\mathcal{D}_{\mathbf{x}}} d^{n} z_{\mathbf{x}} f_{i} g_{i}
$$

with $f$ and $g$ indicating two $N$-tuples $f=\left(f_{1}\left(\mathbf{z}_{\mathbf{x}, \mathbf{v}}\right), \cdots, f_{M}\left(\mathbf{z}_{\mathbf{x}, \mathbf{v}}\right), f_{M+1}\left(\mathbf{z}_{\mathbf{x}}\right), \cdots, f_{N}\left(\mathbf{z}_{\mathbf{x}}\right)\right)$ and $g=\left(g_{1}\left(\mathbf{z}_{\mathbf{x}, \mathbf{v}}\right), \cdots, g_{M}\left(\mathbf{z}_{\mathbf{x}, \mathbf{v}}\right), g_{M+1}\left(\mathbf{z}_{\mathbf{x}}\right), \cdots, g_{N}\left(\mathbf{z}_{\mathbf{x}}\right)\right)$.

In Eq. (14) we indicated with $\delta F / \delta \chi$ the $N$-tuple

$$
\frac{\delta F}{\delta \chi}=\left(\frac{\delta F}{\delta \chi_{1}\left(\mathbf{z}_{\mathbf{x}, \mathbf{v}}\right)}, \cdots, \frac{\delta F}{\delta \chi_{M}\left(\mathbf{z}_{\mathbf{x}, \mathbf{v}}\right)}, \frac{\delta F}{\delta \chi_{M+1}\left(\mathbf{z}_{\mathbf{x}}\right)}, \cdots, \frac{\delta F}{\delta \chi_{N}\left(\mathbf{z}_{\mathbf{x}}\right)}\right),
$$

where $\delta F / \delta \chi_{i}\left(\mathbf{z}_{(i)}\right)$ is the functional derivative of the functional $F$, with respect to the field $\chi_{i}$, at the point $\mathbf{z}_{(i)}$ (at a fixed time $t$ ). The functional derivative is defined from the following 
expression

$$
\lim _{\epsilon \rightarrow 0} \frac{F\left(\chi_{1}, \cdots, \chi_{i}+\epsilon \delta \chi_{i}, \cdots, \chi_{N}\right)-F\left(\chi_{1}, \cdots, \chi_{i}, \cdots, \chi_{N}\right)}{\epsilon} \equiv \int_{\mathcal{D}_{(i)}} d^{n_{(i)}} z_{(i)} \frac{\delta F}{\delta \chi_{i}\left(\mathbf{z}_{(i)}\right)} \delta \chi_{i},
$$

where $\delta \chi_{i}=\delta \chi_{i}\left(\mathbf{z}_{(i)}\right)$ is an arbitrary perturbation vanishing at the boundaries of $\mathcal{D}_{(i)}$. Note that in the above definition we have omitted the explicit dependence of the fields and of the perturbations on the time $t$, which here plays the role of a fixed parameter.

As an example, consider the above mentioned one-dimensional Vlasov-Ampère system, with the space of functions $P$ consisting of the pairs $(f, E)$, where both $f$ and $E$ possess periodic boundary conditions over $[0,1]$ for the spatial coordinate $x$, and where $f$ vanishes for $|v| \rightarrow \infty$. We consider then, as example, the functional (of no particular physical meaning)

$$
F(f, E)=\int_{0}^{1} d x \int_{\mathbb{R}} d v f E+\frac{1}{2} \int_{0}^{1} d x\left(\frac{\partial E}{\partial x}\right)^{2} .
$$

According to the expression (17), obtaining the functional derivative with respect to $E(x)$, requires determining

$$
\lim _{\epsilon \rightarrow 0} \frac{F(f, E+\epsilon \delta E)-F(f, E)}{\epsilon}=\int_{0}^{1} d x \int_{\mathbb{R}} d v f(x, v, t) \delta E(x, t)+\int_{0}^{1} d x \frac{\partial E}{\partial x} \frac{\partial \delta E}{\partial x} .
$$

In order to cast the latter expression in the form of the right-hand side of Eq. (17), we can perform an integration by part on the last term of Eq. (19) (taking into account that boundary terms vanish) and then rewrite the resulting expression as

$$
\lim _{\epsilon \rightarrow 0} \frac{F(f, E+\epsilon \delta E)-F(f, E)}{\epsilon}=\int_{0}^{1} d x\left(\int_{\mathbb{R}} d v f-\frac{\partial^{2} E}{\partial x^{2}}\right) \delta E .
$$

From Eq. (20) we can then extract the expression for the functional derivative, which reads

$$
\frac{\delta F}{\delta E(x, t)}=\int_{\mathbb{R}} d v f(x, v, t)-\frac{\partial^{2} E}{\partial x^{2}}(x, t)
$$

In (21) we have made explicit the dependence on the coordinates as well as on time, although we stress again that, in evaluating the functional derivatives, $t$ plays the role of a fixed parameter. From the definition, it follows also that the functional derivative of $F$ with respect to $f(x, v)$ is given by

$$
\frac{\delta F}{\delta f(x, v, t)}=E(x, t)
$$


A fundamental relation which follows from Eqs. (11) and (17) is given by

$$
\frac{\delta \chi_{i}\left(\mathbf{z}_{(i)}, t\right)}{\delta \chi_{j}\left(\mathbf{z}_{(j)}^{\prime}, t\right)}=\delta_{i j} \delta\left(\mathbf{z}_{(j)}^{\prime}-\mathbf{z}_{(i)}\right) .
$$

A further fundamental tool concerning functional derivatives is the chain rule that is employed when it is needed to express a functional derivative in terms of a new set of variables. Let us consider a functional $\bar{F}(\bar{\chi})$ expressed in terms of a new set of field variables $\bar{\chi}=\left(\bar{\chi}_{1}\left(\mathbf{z}_{\mathbf{x}, \mathbf{v}}\right), \cdots, \bar{\chi}_{M^{\prime}}\left(\mathbf{z}_{\mathbf{x}, \mathbf{v}}\right), \bar{\chi}_{M^{\prime}+1}\left(\mathbf{z}_{\mathbf{x}}\right), \cdots, \bar{\chi}_{N^{\prime}}\left(\mathbf{z}_{\mathbf{x}}\right)\right) \in P^{\prime}$. The elements of the space of functions $P^{\prime}$ are $N^{\prime}$-uples with $M^{\prime}$ functions depending on $\mathbf{z}_{\mathbf{x}, \mathbf{v}}$ and $N^{\prime}-M^{\prime}$ functions depending on $\mathbf{z}_{\mathbf{x}}$, and, in general $M \neq M^{\prime}$ and $N \neq N^{\prime}$ (the case where $P \neq P^{\prime}$ will be the case treated in Sec. V, where it will be required to change from the space of distribution functions to the space of moments). The old variables $\chi$ and the new variables $\bar{\chi}$ are assumed to be related to each other by

$$
\bar{\chi}_{i}=\mathcal{O}^{i j} \chi_{j}, \quad i=1, \cdots, N^{\prime}
$$

where $\mathcal{O}^{i j}$ are the components of a, possibly nonlinear and noninvertible operator $\mathcal{O}: P \rightarrow$ $P^{\prime}$. We indicate by $F(\chi)$ the functional $\bar{F}$ expressed in terms of the old variables. Scalar invariance clearly implies

$$
F(\chi)=\bar{F}(\bar{\chi})
$$

and

$$
\left\langle\frac{\delta F}{\delta \chi} \mid \delta \chi\right\rangle=\left\langle\frac{\delta \bar{F}}{\delta \bar{\chi}} \mid \delta \bar{\chi}\right\rangle^{\prime},
$$

where the prime on the right-hand side indicates that the inner product is taken as defined in Eq. (15) but with $M$ and $N$ replaced by $M^{\prime}$ and $N^{\prime}$, respectively. In Eq. (26), the variations $\delta \chi=\left(\delta \chi_{1}, \cdots, \delta \chi_{N}\right)$ and $\delta \bar{\chi}=\left(\delta \bar{\chi}_{1}, \cdots, \delta \bar{\chi}_{N}\right)$ are related by

$$
\delta \bar{\chi}_{i}=O^{i j} \delta \chi_{j}, \quad i=1, \cdots, N^{\prime}
$$

where $O^{i j}$ indicate the components of a now linear operator $O: P \rightarrow P^{\prime}$, obtained from the linearization of Eq. (24) for small variations $\delta \bar{\chi}$ of $\bar{\chi}$ and $\delta \chi$ of $\chi$. Upon using Eq. (27) and Eq. (26) one obtains

$$
\left\langle\frac{\delta \bar{F}}{\delta \bar{\chi}} \mid O \delta \chi\right\rangle^{\prime}=\left\langle O^{\dagger} \frac{\delta \bar{F}}{\delta \bar{\chi}} \mid \delta \chi\right\rangle,
$$


where $O^{\dagger}: P^{\prime} \rightarrow P$ is the adjoint of the operator $O$. From Eqs. (26) and (28), it follows then that

$$
\frac{\delta F}{\delta \chi}=O^{\dagger} \frac{\delta \bar{F}}{\delta \bar{\chi}},
$$

which expresses the chain rule relating the functional derivatives with respect to the old variables, to those with respect to the new variables.

In order to illustrate further properties that will be used thorughout the article, we note that, from Eqs. (10), (14) and (23), it follows that, for a Hamiltonian system, the evolution equation for the $i$ th field can be written as

$$
\frac{\partial \chi_{i}}{\partial t}=J^{i j} \frac{\delta H}{\delta \chi_{j}},
$$

where summation over the repeated index $j$ is understood, and where we indicate by $J^{i j}$ the components of the matrix operator $J$. By using the chain rule and integrating by parts one obtains that, for functionals of the form (13), the following relations hold

$$
\begin{aligned}
& \frac{\partial F}{\partial t}=\sum_{i=1, \cdots, M} \int_{\mathcal{D}_{\mathbf{x}, \mathbf{v}}} d^{m+n} z_{\mathbf{x}, \mathbf{v}} \frac{\delta F}{\delta \chi_{i}} \frac{\partial \chi_{i}}{\partial t}+\sum_{\substack{i=M+1, \cdots, N \\
\text { i=1, }}} \int_{\mathcal{D}_{\mathbf{x}}} d^{n} z_{\mathbf{x}} \frac{\delta F}{\delta \chi_{i}} \frac{\partial \chi_{i}}{\partial t} \\
& =\sum_{\mathcal{D}_{\mathbf{x}, \mathbf{v}}} d^{m+n} z_{\mathbf{x}, \mathbf{v}} \frac{\delta F}{\delta \chi_{i}} J^{i j} \frac{\delta H}{\delta \chi_{j}}+\sum_{\substack{i=M+1, \cdots, N \\
j=1, \cdots, N}} \int_{\mathcal{D}_{\mathbf{x}}} d^{n} z_{\mathbf{x}} \frac{\delta F}{\delta \chi_{i}} J^{i j} \frac{\delta H}{\delta \chi_{j}} \\
& =\{F, H\},
\end{aligned}
$$

where, for the last step, we made use of Eq. (30). From Eq. (31), and from the antisymmetry of the Poisson bracket, it follows then that

$$
\frac{\partial H}{\partial t}=\{H, H\}=0
$$

which expresses the fundamental fact that, for Hamiltonian systems, the Hamiltonian (functional, in this context) is a conserved quantity, reflecting the conservation of the total energy of the system.

Having introduced basic definitions and tools for Hamiltonian continuum models, the natural question, relevant to our context, could be : when does a given model of the form (9) possess a Hamiltonian structure? In general, no systematic procedure exists to answer 
such question, which is what makes the subject complex and, at the same time, interesting. Related to this question are the remarks that follow, with which we touch upon an important feature characterizing the Hamiltonian structures of fluid models for plasmas, i.e. their noncanonical Poisson brackets.

We recall that, according to the above definition of Hamiltonian system, the Hamiltonian structure for system of evolution equations of the form (9), for a certain set of field variables, consists of two elements: the Hamiltonian functional and the Poisson bracket. In the problem of identifying the Hamiltonian structure of a (possibly Hamiltonian) given model, Eq. (32) implies that the Hamiltonian functional has to be sought for among the quantities conserved by that model. Because the Hamiltonian expresses the total conserved energy of a physical model, an amount of physical intuition could help in identifying the Hamiltonian functional of a given model. The identification of the corresponding Poisson bracket, on the other hand, can be a much less intuitive process, in general. Indeed, one could consider the canonical Poisson brackets for field theories. For instance, assuming $M=0, N=2$ and $\mathcal{D}_{\mathbf{x}}=\mathbb{R}^{3}$ as domain, one can consider the Poisson operator (which, in such nondegenerate case, is also referred to as cosymplectic form)

$$
J=\left(\begin{array}{cc}
0 & 1 \\
-1 & 0
\end{array}\right)
$$

leading to the canonical Poisson bracket

$$
\{F, G\}=\int_{\mathbb{R}^{3}} d^{3} x\left(\frac{\delta F}{\delta \chi_{1}} \frac{\delta G}{\delta \chi_{2}}-\frac{\delta F}{\delta \chi_{2}} \frac{\delta G}{\delta \chi_{1}}\right)
$$

At each point $\mathbf{x} \in \mathbb{R}^{3}$, the field $\chi_{1}$ is said to be canonically conjugate to the field $\chi_{2}$. Note that, the discrete version of Eq. (34), where fields are replaced by $2 n$ functions of time $\left(q_{1}, \cdots, q_{n}, p_{i}, \cdots, p_{n}\right)$ writes

$$
\{f, g\}=\sum_{i=1}^{n} \frac{\partial f}{\partial q_{i}} \frac{\partial g}{\partial p_{i}}-\frac{\partial f}{\partial p_{i}} \frac{\partial g}{\partial q_{i}}
$$

for two functions $f=f\left(q_{1}, \cdots, p_{n}\right), g=g\left(q_{1}, \cdots, p_{n}\right)$. The operation (35) corresponds to the Poisson bracket used in classical mechanics to describe the evolution of the positions 
$q_{1}, \cdots, q_{n}$ of $n$ particles and of the corresponding canonically conjugate momenta $p_{1}, \cdots, p_{n}$, in the case when particles are subject to a potential depending on the positions.

It is relatively easy to verify that the operation (34) satisfies the above four properties of a Poisson bracket. On the other hand, the set of operations satisfying such properties is much larger, Indeed, as assumed above, the Poisson operator $J$ can in principle depend on the fields, their derivatives and the coordinates. In particular, Poisson brackets can exist, for which it is not possible to identify, globally on the phase space, pairs of canonically conjugate variables, as it happens in canonical field theories, or, at the discrete level, in the above example encountered in classical mechanics. It turns out that, indeed, Hamiltonian continuum theories for plasmas formulated from the Eulerian point of view, such as those treated in this article, are characterized by noncanonical Poisson brackets. The origin for the appearance of such a noncanonical Hamiltonian structure resides in the nature of the transformation mapping the fields expressed in terms of Lagrangian coordinates to the fields in Eulerian coordinates. This transformation maps the canonical Poisson brackets expressed in terms of Lagrangian coordinates, to the noncanonical Poisson brackets written in Eulerian coordinates, and provides an example of reduction by symmetry. The subject is described in detail in Refs. [38, 39, 51-53]. In Sec. II B 1 the reduction from the canonical Poisson bracket in Lagrangian coordinates to the noncanonical Poisson bracket in Eulerian coordinates will be reviewed in the case of a barotropic fluid.

Given their noncanonical nature, the identification of Poisson brackets for fluid or kinetic plasma models can in general be a difficult task, in particular as far as the the verification of the Jacobi identity is concerned.

One of the main features of noncanonical Hamiltonian systems is that the associated dynamics takes place on surfaces, denoted as symplectic leaves, that foliate the phase space. The existence of symplectic leaves is associated with the presence of so-called Casimir invariants, characterizing noncanonical Hamiltonian systems. In our context, the phase space of a Hamiltonian continuum model corresponds to the above mentioned space of functions 
$P$. Casimir invariants are elements $C\left(\chi_{1}, \cdots, \chi_{N}\right) \in \mathcal{F}(P)$ such that

$$
\{C, F\}=0, \quad \forall F \in \mathcal{F}(P) .
$$

Thus, Casimir invariants commute, through the Poisson bracket, with any other functional of the field variables. In particular they commute with the Hamiltonian functional. From Eq. (31) it follows then that, for a Casimir invariant $C$

$$
\frac{\partial C}{\partial t}=\{C, H\}=0
$$

which proves the fact that $C$ is an invariant of motion.

The existence of Casimir invariants is related to the property of Poisson operators of noncanonical Poisson brackets of possessing a non trivial kernel. It can be seen, on the other hand, that a canonical Poisson bracket such as (34), only possesses trivial invariants, not depending on the field variables.

For applications, the importance of Casimir invariants for fluid and kinetic models is associated mainly with the constraints that they impose on the dynamics and on the possibility they offer for investigating equilibria and stability [38, 39].

\section{B. Example: barotropic fluid}

Before moving to Hamiltonian fluid models for plasmas, we find it convenient to consider a Hamiltonian model of fluid mechanics, in order to illustrate with an example the concepts previously introduced in this Section. More precisely we will describe the Hamiltonian structure of the non dissipative equations for a three-dimensional barotropic fluid, which are given by

$$
\begin{aligned}
& \frac{\partial \rho}{\partial t}=-\nabla \cdot(\rho \mathbf{v}) \\
& \frac{\partial \mathbf{v}}{\partial t}=-(\mathbf{v} \cdot \nabla) \mathbf{v}-\frac{1}{\rho} \nabla p(\rho)
\end{aligned}
$$

In Eqs. (38)-(39) $\rho(\mathbf{x}, t)$ is the mass density, $\mathbf{v}(\mathbf{x}, t)$ is the velocity field and $p(\rho)$ is the pressure, which, for a barotropic fluid, only depends on the density. Note that, with a slight 
abuse of notation, here we indicated with $\mathbf{v}$ the velocity vector field which is a function of $\mathbf{x}$ and $t$. The same symbol is used in the present article also to indicate a point in the particle velocity coordinate space. Depending on the context, we think it should be possible to easily distinguish which is the meaning of the symbol, without leading to confusion. Equations (38) and (39) represent the continuity equation and the evolution equation for the velocity vector field. With reference to the framework described following Eq. (9), the model (38)-(39) corresponds to the situation where $N=4$, with the four field variables given by $\chi_{1}=\rho, \chi_{2}=v_{x}, \chi_{3}=v_{y}, \chi_{4}=v_{z}$, with $v_{x}, v_{y}$ and $v_{z}$ indicating the three components of the velocity vector field. All four fields depend on spatial coordinates and not on velocity coordinates, therefore we are in the case $M=0$, and we consider the situation where $\mathbf{z}_{\mathbf{x}}=(x, y, z) \in \mathcal{D}_{\mathbf{x}} \equiv[0,1] \times[0,1] \times[0,1]$. We assume also that the fields behave at the boundaries of $\mathcal{D}_{\mathbf{x}}$ in such a way that boundary terms vanish when integrating by parts (an hypothesis that will be tacitly assumed throughout the whole article). The elements of the space of functions $P$, in this case, will consist then of 4 -uples $(\rho, \mathbf{v})$ defined over $\mathcal{D}_{\mathbf{x}}$ and satisfying the prescribed boundary conditions.

The model (38)-(39) possesses a Hamiltonian structure (see, e.g. Refs. [38, 49]) consisting of the Hamiltonian functional

$$
H(\rho, \mathbf{v})=\int_{\mathcal{D}_{\mathbf{x}}} d^{3} x\left(\frac{\rho}{2} v^{2}+\rho U(\rho)\right)
$$

and of the Poisson bracket

$$
\{F, G\}=-\int_{\mathcal{D}_{\mathbf{x}}} d^{3} x\left(F_{\rho} \nabla \cdot G_{\mathbf{v}}-G_{\rho} \nabla \cdot F_{\mathbf{v}}+\frac{\nabla \times \mathbf{v}}{\rho} \cdot G_{\mathbf{v}} \times F_{\mathbf{v}}\right)
$$

where the subscripts over functionals indicate functional derivatives, so that, for instance, $F_{\rho} \equiv \delta F / \delta \rho$

In Eq. (40) we indicated with $U(\rho)$ the internal energy per unit mass of the fluid, a prescribed function of the mass density, which, in general, is related to the pressure by

$$
p=\rho^{2} \frac{\partial U}{\partial \rho} .
$$


In more general discussions of barotropic fluids, such as those of Refs. [38, 50], the internal energy is assumed to depend also on the specific entropy. In the context of the present example we prefer to simplify the model and omit the dependence on the specific entropy. Such dependence, on the other hand, will be included in the adiabatic and double adiabatic MHD models discussed in Secs. III A and III C.

It can be verified that the functional $H$ is conserved by Eqs. (38)-(39). Indeed, such functional represents the total energy of the fluid given by the sum of the kinetic and of the internal energy, corresponding to the first and second term on the right-hand side of Eq. (40), respectively.

The functional derivatives of $H$ with respect to the field variables can be calculated to give

$$
H_{\rho}=\frac{v^{2}}{2}+U+\rho U^{\prime}, \quad H_{\mathbf{v}}=\rho \mathbf{v},
$$

where the prime denotes derivative with respect to the argument of the function.

Making use of Eq. (23) and of integration by parts one can then see that the model equations (38)-(39) can be written in the Hamiltonian form

$$
\frac{\partial \rho}{\partial t}=\{\rho, H\}, \quad \frac{\partial \mathbf{v}}{\partial t}=\{\mathbf{v}, H\},
$$

with $H$ and $\{$,$\} given by Eqs. (40) and (41), respectively.$

Clearly, a fundamental point concerns the capability of the bracket (41) of satisfying the four properties of a Poisson bracket. Whereas it is rather straightforward to show that the bracket (41) satisfies bilinearity, antisymmetry and the Leibniz identity, a much less trivial point concerns the Jacobi identity. Although strategies exist in order to derive Poisson brackets for Hamiltonian models (see, e.g. Refs. [37-39, 54, 55]), for a good fraction of the Hamiltonian models reviewed in this article ansätze for the Hamiltonian functional and the Poisson bracket were proposed, and the properties of the Poisson bracket were checked a posteriori. In particular, as far as the verification of the Jacobi identity is concerned, a result presented in Ref. [56], greatly simplifies the, otherwise usually extremely lengthy, calculations. Indeed, from the expression of the Jacobi identity (iv) and from Eq. (14), 
it emerges that the direct verification of the Jacobi identity requires calculating functional derivatives of the form

$$
\frac{\delta\{F, G\}}{\delta \chi}=\frac{\delta}{\delta \chi}\left(\sum_{\substack{i=1, \cdots, M \\ j=1, \cdots, N}} \int_{\mathcal{D}_{\mathbf{x}, \mathbf{v}}} d^{m+n} z_{\mathbf{x}, \mathbf{v}} \frac{\delta F}{\delta \chi_{i}} J^{i j} \frac{\delta G}{\delta \chi_{j}}+\sum_{\substack{i=M+1, \cdots, N \\ j=1, \cdots, N}} \int_{\mathcal{D}_{\mathbf{x}}} d^{n} z_{\mathbf{x}} \frac{\delta F}{\delta \chi_{i}} J^{i j} \frac{\delta G}{\delta \chi_{j}}\right) .
$$

The Lemma proved in Ref. [56] implies that, when evaluating the functional derivatives of the form (45) in the expression $\{F,\{G, H\}\}+\{G,\{H, F\}\}+\{H,\{F, G\}\}$, only the variations due to the dependence of $J$ on the field variables have to be taken into account. The remaining contributions (i.e., in the case of Eq. (45), those due to the dependencies of $F$ and $G$ on the field variables) indeed cancel each other, due to the skew-symmetry of $J$ (which is required by the antisymmetry of the Poisson bracket).

Further simplifications in the verification of the Jacobi identity can occur if one is able to find a new set of field variables, in terms of which the Poisson bracket takes a simplified form. If the change of variables is invertible, verifying the Jacobi identity for the bracket in its simplified form, is equivalent to verifiying it in its form expressed in terms of the original set of variables. In this example, a natural invertible (given that $\rho>0$ ) change of variables is the following:

$$
\bar{\rho}=\rho, \quad \mathbf{M}=\rho \mathbf{v},
$$

where the new variables are $(\bar{\rho}, \mathbf{M})$ and where, in particular, we replaced the velocity field with the momentum density M. Making use of the functional chain rule (29) we obtain the expressions

$$
\frac{\delta F}{\delta \mathbf{v}}=\bar{\rho} \frac{\delta \bar{F}}{\delta \mathbf{M}}, \quad \frac{\delta F}{\delta \rho}=\frac{\mathbf{M}}{\bar{\rho}} \cdot \frac{\delta \bar{F}}{\delta \mathbf{M}}+\frac{\delta \bar{F}}{\delta \bar{\rho}},
$$

which, when inserted into (41), upon making use of vector identities, yield

$$
\{F, G\}=-\int_{\mathcal{D}_{\mathbf{x}}} d^{3} x\left(\rho\left(F_{\mathbf{M}} \cdot \nabla G_{\rho}-G_{\mathbf{M}} \cdot \nabla F_{\rho}\right)+\mathbf{M} \cdot\left(F_{\mathbf{M}} \cdot \nabla G_{\mathbf{M}}-G_{\mathbf{M}} \cdot \nabla F_{\mathbf{M}}\right)\right) .
$$

In Eq. (48) we omitted the overbar symbol on the quantities related to the new field variables. The expression (48) corresponds to the Poisson bracket for a barotropic fluid but expressed in terms of mass and momentum densities. In the Appendix A we show how 
the verification of the Jacobi identity proceeds for such bracket (and consequently for the bracket (41)). We remark that, in terms of the new variables, the Hamiltonian (40) becomes

$$
H(\rho, \mathbf{M})=\int_{\mathcal{D}_{\mathbf{x}}} d^{3} x\left(\frac{M^{2}}{2 \rho}+\rho U(\rho)\right),
$$

and the model equations generated by the bracket (48) and the Hamiltonian (49) read

$$
\begin{aligned}
& \frac{\partial \rho}{\partial t}=-\nabla \cdot \mathbf{M} \\
& \frac{\partial \mathbf{M}}{\partial t}=-\nabla \cdot\left(\mathbf{M} \frac{\mathbf{M}}{\rho}+p \mathbf{I}\right)
\end{aligned}
$$

where $\mathbf{I}$ is the identity matrix.

To conclude, we remark that, starting from the definition (36) and using integration by parts, Casimir invariants $C$ for the barotropic fluid bracket (41) have to satisfy

$$
\nabla \cdot C_{\mathbf{v}}=0, \quad \nabla C_{\rho}+\frac{\nabla \times \mathbf{v}}{\rho} \times C_{\mathbf{v}}=0 .
$$

Solutions of this system are given by

$$
C_{1}=\int_{\mathcal{D}_{\mathbf{x}}} d^{3} x \rho, \quad C_{2}=\int_{\mathcal{D}_{\mathbf{x}}} d^{3} x \mathbf{v} \cdot \nabla \times \mathbf{v},
$$

which correspond to the total mass and to the total helicity, two quantities which are indeed well known to be conserved by an inviscid barotropic fluid.

1. Derivation of the Eulerian noncanonical Poisson bracket from the canonical Poisson bracket in the Lagrangian description

As mentioned above in Sec. II, the noncanonical character of Poisson brackets for fluid theories expressed from the Eulerian viewpoint, emerges as a consequence of a reduction process occurring when moving from the canonical description in terms of Lagrangian variables to the description in terms of Eulerian variables.

We illustrate how this process occurs in the above treated case of a barotropic fluid. The following description includes also the derivation of the fluid equations of motion from an action principle. The subject is described in various references such as Refs. [38, 51]. 
We find it useful to introduce the subject by recalling basic notions about canonical Hamiltonian systems with a finite number of degrees of freedom.

As is well known, the canonical equations for a Hamiltonian system with $\mathcal{N}$ degrees of freedom, are given by

$$
\dot{q}_{i}=\frac{\partial H}{\partial p_{i}}, \quad \dot{p}_{i}=-\frac{\partial H}{\partial q_{i}}, \quad i=1, \cdots, \mathcal{N}
$$

and can be expressed in the form

$$
\dot{q}_{i}=\left\{q_{i}, H\right\}, \quad \dot{p}_{i}=\left\{p_{i}, H\right\}, \quad i=1, \cdots, \mathcal{N},
$$

where $H(q, p)$ is the Hamiltonian function (where we introduced the symbols $q=\left(q_{1}, \cdots, q_{\mathcal{N}}\right), p=$ $\left.\left(p_{1}, \cdots, p_{\mathcal{N}}\right)\right)$ and $\{$,$\} is the canonical Poisson bracket defined as$

$$
\{f, g\}=\sum_{i=1}^{\mathcal{N}} \frac{\partial f}{\partial q_{i}} \frac{\partial g}{\partial p_{i}}-\frac{\partial f}{\partial p_{i}} \frac{\partial g}{\partial q_{i}}
$$

for two functions $f$ and $g$.

Eqs. (54) can be obtained from the $\mathcal{N}$, second-order Lagrange's equations

$$
\frac{d}{d t} \frac{\partial L}{\partial \dot{q}_{i}}=\frac{\partial L}{\partial q_{i}}, \quad i=1, \cdots, \mathcal{N}
$$

which, in turn, are obtained as extremals of the action functional

$$
S[q]=\int_{t_{0}}^{t_{1}} d t L(q, \dot{q})
$$

In (57) and (58), the function $L(q, \dot{q})$ is the Lagrangian of the system, where $t_{0}$ and $t_{1}$ indicate two instants of time. The notation $S[q]$ is to indicate that the value of the action $S$ depends on a path $q$. This path must belong to the class of curves $q(t)$ such that $q\left(t_{0}\right)=\bar{q}_{0}$ and $q\left(t_{1}\right)=\bar{q}_{1}$, with $\bar{q}_{0}$ and $\bar{q}_{1}$ fixed values of $q$ determining the end conditions.

For physical systems, the Lagrangian function usually takes the form

$$
L=T-V
$$

where $T$ and $V$ indicate the kinetic and potential energy of the system, respectively. 
The canonical Hamilton's equations (54) can be obtained from Lagrange's equations by introducing the canonical momenta

$$
p_{i}=\frac{\partial L}{\partial \dot{q}_{i}}, \quad i=1, \cdots, \mathcal{N}
$$

and the Hamiltonian function $H$ as Legendre transform of the Lagrangian in the following way (provided one is able to express $\dot{q}$ in terms of $q$ and $p$, which leads to the issue of the convexity of the Lagrangian $L$ with respect to the variable $\dot{q}$ ):

$$
H(q, p)=\sum_{i=1}^{\mathcal{N}} p_{i} \dot{q}_{i}-L(q, \dot{q}) .
$$

The above construction can be extended to the case of a field theory, in which the Lagrangian becomes a functional of field variables. In the context of fluid models, this is the case when the fluid motion is described in terms of Lagrangian variables. In the Eulerian description, adopted for the models treated in this review, field variables are functions of coordinates fixed in space. The fluid motion is then described by measuring how quantities vary in time at fixed positions in space. According to the Lagrangian viewpoint, on the other hand, the fluid motion is described in terms of trajectories of the flow $\mathbf{q}=\mathbf{q}(\mathbf{a}, t)$, which gives the position, at time $t$, of the infinitesimal fluid element, which was at the position a at $t=0$ (the condition $\mathbf{q}(\mathbf{a}, 0)=\mathbf{a}$ is thus implied).

The map $\mathbf{q}: \mathcal{D}_{\mathbf{x}} \rightarrow \mathcal{D}_{\mathbf{x}}$ is assumed to be invertible and differentiable as many times as required.

The evolution of the trajectories of the infinitesimal fluid parcels is determined by equations of motion that can be obtained as Lagrange's equations following from a variational principle, as above described. However, in this case the dynamical variables $\mathbf{q}$ depend on the continuous label $\mathbf{a} \in \mathcal{D}_{\mathbf{x}}$. Consequently, they are treated as field variables and the Lagrangian takes the form of a functional. Such functional, however, in the case of a barotropic fluid, is still of the form (59). In particular, the kinetic and potential energy contributions $T$ and $V$ correspond to the first and second term of Eq. (49), although expressed in Lagrangian variables. We proceed then namely with expressing $T$ and $V$ in Lagrangian variables, beginning with the latter. 
To this purpose, we introduce the relation

$$
\rho(\mathbf{x}, t)=\int_{\mathcal{D}_{\mathbf{x}}} d^{3} a \delta(\mathbf{q}(\mathbf{a}, t)-\mathbf{x}) \rho_{0}(\mathbf{a}),
$$

which permits to express the mass density in the Eulerian representation, in terms of Lagrangian variables. In Eq. (62), $\rho_{0}(\mathbf{a})$ indicates the distribution of mass density at $t=0$ and attributes a value of density to each value of the label a associated with an infinitesimal fluid element.

The relation (62) can be written in another remarkable form. A property of the Dirac delta is indeed that

$$
\delta(\mathbf{q}(\mathbf{a}, t)-\mathbf{x})=\frac{\delta\left(\mathbf{a}-\mathbf{q}^{-1}(\mathbf{x}, t)\right)}{\left.\mathcal{J}(\mathbf{a}, t)\right|_{\mathbf{a}=\mathbf{q}^{-1}(\mathbf{x}, t)}},
$$

where $\mathcal{J}(\mathbf{a}, t)=\operatorname{det}\left(\partial q_{i} / \partial a_{j}\right)$ is the determinant of the Jacobian of the map $\mathbf{q}(\mathbf{a}, t)$ at a fixed $t$. Inserting (63) into (62) yields

$$
\rho(\mathbf{x}, t)=\left.\frac{\rho_{0}(\mathbf{a})}{\mathcal{J}(\mathbf{a}, t)}\right|_{\mathbf{a}=\mathbf{q}^{-1}(\mathbf{x}, t)} .
$$

Because $\mathcal{J}$ is the Jacobian of the transformation $\mathbf{q}(\mathbf{a})$, a volume element $d^{3} a$ is mapped into a volume element $d^{3} x=d^{3} q=\mathcal{J} d^{3} a$. Using this relation in (64) yields

$$
\rho(\mathbf{x}, t) d^{3} x=\rho_{0}(\mathbf{a}) d^{3} a,
$$

which, correctly implies that the total fluid mass $\int_{\mathcal{D}_{\mathbf{x}}} d^{3} x \rho(\mathbf{x}, t)$ remains constant. Using (64), we can rewrite the potential energy in terms of the Lagrangian variables $\mathbf{q}(\mathbf{a}, t)$ (and possibly of their derivatives with respect to time or to the parameter a). Clearly, the coordinates $\mathbf{x}$ will not appear in this representation, and the integrals will be taken with respect to the parameter a instead. Thus, we obtain

$$
V(\mathbf{q})=\int_{\mathcal{D}_{\mathbf{x}}} d^{3} a \rho_{0}(\mathbf{a}) U\left(\rho_{0} / \mathcal{J}\right) .
$$

Note that the dependence on $\mathbf{q}$ enters through the dependence of $\mathcal{J}$ on $\partial q_{i} / \partial a_{j}$.

Concerning the kinetic energy, we note that the Eulerian velocity field is related to the flow $\mathbf{q}$ by 


$$
\mathbf{v}(\mathbf{x}, t)=\left.\dot{q}(\mathbf{a}, t)\right|_{\mathbf{a}=\mathbf{q}^{-1}(\mathbf{x}, t)} .
$$

Consequently, the kinetic energy in Lagrangian variables reads:

$$
T(\mathbf{q})=\frac{1}{2} \int_{\mathcal{D}_{\mathbf{x}}} d^{3} a \rho_{0} \dot{q}^{2}
$$

The Lagrangian functional then reads

$$
L(\mathbf{q}, \dot{\mathbf{q}})=\int_{\mathcal{D}_{\mathbf{x}}} d^{3} a\left(\frac{1}{2} \rho_{0} \dot{q}^{2}-\rho_{0}(\mathbf{a}) U\left(\rho_{0} / \mathcal{J}\right)\right),
$$

and the action is

$$
S[\mathbf{q}]=\int_{t_{0}}^{t_{1}} d t \int_{\mathcal{D}_{\mathbf{x}}} d^{3} a\left(\frac{1}{2} \rho_{0} \dot{q}^{2}-\rho_{0} U\left(\rho_{0} / \mathcal{J}\right)\right) .
$$

We are now in the position for deriving Lagrange's equations from the action (70) following the procedure described above for a finite-dimensional system.

More precisely, Lagrange's equations can be found as extremals of $S$, by setting its first variation $\delta S$ equal to zero:

$$
\begin{aligned}
& \delta S(\mathbf{q} ; \delta \mathbf{q}) \equiv \int_{t_{0}}^{t_{1}} d t \int_{\mathcal{D}_{\mathbf{x}}} d^{3} a \frac{\delta S}{\delta q_{i}(\mathbf{a}, t)} \delta q_{i}(\mathbf{a}, t) \\
& =\int_{t_{0}}^{t_{1}} d t \int_{\mathcal{D}_{\mathbf{x}}} d^{3} a \delta q_{l}\left[-\rho_{0} \ddot{q}_{l}-A_{l}^{p} \frac{\partial}{\partial a_{p}}\left(\frac{\rho_{0}^{2}}{\mathcal{J}^{2}} \frac{\partial U}{\partial\left(\frac{\rho_{0}}{\mathcal{J}}\right)}\right)\right],
\end{aligned}
$$

where sum over repeated indices is understood and where $A_{l}^{p}=(1 / 2) \epsilon_{l r s} \epsilon_{p m n}\left(\partial q_{r} / \partial a_{m}\right)\left(\partial q_{s} / \partial a_{n}\right)$ is the cofactor of the matrix element $\partial q_{l} / \partial a_{p}$. For obtaining (72), we made use of the relations

$$
\frac{\partial \mathcal{J}}{\partial\left(\frac{\partial q_{i}}{\partial a_{j}}\right)}=A_{i}^{j}
$$

and

$$
\frac{\partial A_{i}^{j}}{\partial a_{j}}=0, \quad i=1,2,3 .
$$

Lagrange's equations deriving from (72) are then given by

$$
\rho_{0} \ddot{q}_{l}+A_{l}^{p} \frac{\partial}{\partial a_{p}}\left(\frac{\rho_{0}^{2}}{\mathcal{J}^{2}} \frac{\partial U}{\partial\left(\frac{\rho_{0}}{\mathcal{J}}\right)}\right)=0, \quad l=1,2,3 .
$$


After multiplying Eq. (75) times $\partial q_{l} / \partial a_{i}$ and using the relation

$$
\frac{\partial q_{l}}{\partial a_{i}} A_{l}^{p}=\mathcal{J} \delta_{i}^{p}
$$

one obtains

$$
\rho_{0} \ddot{q}_{l} \frac{\partial q_{l}}{\partial a_{i}}+\mathcal{J} \frac{\partial}{\partial a_{i}}\left(\frac{\rho_{0}^{2}}{\mathcal{J}^{2}} \frac{\partial U}{\partial\left(\frac{\rho_{0}}{\mathcal{J}}\right)}\right)=0,
$$

which is the dynamical equation for a 3D adiabatic fluid in Lagrangian variables. In Eulerian variables, Eq. (77) corresponds to Eq. (39).

The Hamiltonian formulation in Lagrangian variables can then be obtained first by introducing, analogously to Eq. (60), the conjugate momenta

$$
\pi_{i}(\mathbf{a}, t)=\frac{\delta L}{\delta \dot{q}_{i}}=\rho_{0} \dot{q}_{i}, \quad i=1,2,3,
$$

and then performing a Legendre transform. The Hamiltonian functional is obtained by extending Eq. (61) to the continuum case and corresponds to

$$
\begin{aligned}
& H(\boldsymbol{\pi}, \mathbf{q})=\int_{\mathcal{D}_{\mathbf{x}}} d^{3} a\left[\pi_{l} \dot{q}_{l}-\left(\frac{1}{2} \rho_{0} \dot{q}^{2}-\rho_{0}(\mathbf{a}) U\left(\rho_{0} / \mathcal{J}\right)\right)\right] \\
& =\int_{\mathcal{D}_{\mathbf{x}}} d^{3} a\left(\frac{\pi^{2}}{2 \rho_{0}}+\rho_{0} U\right) .
\end{aligned}
$$

The canonical Poisson bracket

$$
\{F, G\}=\int_{\mathcal{D}_{\mathbf{x}}} d^{3} a\left(F_{\mathbf{q}} \cdot G_{\boldsymbol{\pi}}-F_{\boldsymbol{\pi}} \cdot G_{\mathbf{q}}\right),
$$

together with the Hamiltonian (79), yields then the Hamiltonian system

$$
\begin{aligned}
& \dot{q}_{i}=\left\{q_{i}, H\right\}=\frac{\pi_{i}}{\rho_{0}}, \\
& \dot{\pi}_{i}=\left\{\pi_{i}, H\right\}=-A_{i}^{p} \frac{\partial}{\partial a_{p}}\left(\frac{\rho_{0}^{2}}{\mathcal{J}^{2}} \frac{\partial U}{\partial\left(\frac{\rho_{0}}{\mathcal{J}}\right)}\right),
\end{aligned}
$$

for $i=1,2,3$. In particular, from the relation (76), it follows that Eq. (83) is equivalent to Eq. (77). From the Lagrangian viewpoint, then, the field equations describing the dynamics of a barotropic fluid, are a canonical Hamiltonian system in infinite dimensions. As above anticipated, the occurrence of a noncanonical structure in the Poisson bracket (48), emerges 
then as a consequence of the transformation from the Lagrangian to the Eulerian variables. Such transformation is given by

$$
\begin{aligned}
& \rho(\mathbf{x}, t)=\int_{\mathcal{D}_{\mathbf{x}}} d^{3} a \delta(\mathbf{q}(\mathbf{a}, t)-\mathbf{x}) \rho_{0}(\mathbf{a}), \\
& \mathbf{M}(\mathbf{x}, t)=\int_{\mathcal{D}_{\mathbf{x}}} d^{3} a \delta(\mathbf{q}(\mathbf{a}, t)-\mathbf{x}) \boldsymbol{\pi}(\mathbf{a}, t) .
\end{aligned}
$$

Eq. (84) corresponds to the already introduced Equation (62),whereas Eq. (85) relates the fluid momentum $\mathbf{M}(\mathbf{x}, t)$ in Eulerian variables, to the Lagrangian variables $\boldsymbol{\pi}(\mathbf{a}, t)$ and $\mathbf{q}(\mathbf{a}, t)$. The bracket in terms of the Eulerian variables can be obtained, as above explained, by applying the functional chain rule. The variations of the Eulerian fields are given by

$$
\begin{aligned}
& \delta \rho=-\int_{\mathcal{D}_{\mathbf{x}}} d^{3} a \rho_{0}(\mathbf{a}) \frac{\partial}{\partial x_{l}} \delta(\mathbf{x}-\mathbf{q}(\mathbf{a}, t)) \delta q_{l}, \\
& \delta M_{m}=\int_{\mathcal{D}_{\mathbf{x}}} d^{3} a\left(\delta(\mathbf{x}-\mathbf{q}(\mathbf{a}, t)) \delta \pi_{m}-\pi_{m} \frac{\partial}{\partial x_{l}} \delta(\mathbf{x}-\mathbf{q}(\mathbf{a}, t)) \delta q_{l}\right), \quad m=1,2,3,
\end{aligned}
$$

leading to

$$
\begin{aligned}
& \delta F=\int_{\mathcal{D}_{\mathbf{x}}} d^{3} x\left[\left(-\frac{\delta \bar{F}}{\delta \rho} \int_{\mathcal{D}_{\mathbf{x}}} d^{3} a \rho_{0}(\mathbf{a}) \frac{\partial}{\partial x_{l}} \delta(\mathbf{x}-\mathbf{q}(\mathbf{a}, t))\right.\right. \\
& \left.\left.-\frac{\delta \bar{F}}{\delta M_{m}} \int_{\mathcal{D}_{\mathbf{x}}} d^{3} a \pi_{m} \frac{\partial}{\partial x_{l}} \delta(\mathbf{x}-\mathbf{q}(\mathbf{a}, t))\right) \delta q_{l}-\frac{\delta \bar{F}}{\delta M_{m}} \int_{\mathcal{D}_{\mathbf{x}}} d^{3} a \delta(\mathbf{x}-\mathbf{q}(\mathbf{a}, t)) \delta \pi_{m}\right]= \\
& =\int_{\mathcal{D}_{\mathbf{x}}} d^{3} a\left(\frac{\delta F}{\delta q_{i}} \delta q_{i}+\frac{\delta F}{\delta \pi_{s}} \delta \pi_{s}\right),
\end{aligned}
$$

for functionals $F$ and $\bar{F}$ satisfying $F(\mathbf{q}, \boldsymbol{\pi})=\bar{F}(\rho, \mathbf{M})$. The transformation from Lagrangian to Eulerian variables in the functional derivatives gives

$$
\begin{aligned}
& \frac{\delta F}{\delta q_{i}}=\int_{\mathcal{D}_{\mathbf{x}}} d^{3} x\left(\rho_{0} \frac{\partial}{\partial x_{i}} \frac{\delta \bar{F}}{\delta \rho}+\pi_{m} \frac{\partial}{\partial x_{i}} \frac{\delta \bar{F}}{\delta M_{m}}\right) \delta(\mathbf{x}-\mathbf{q}(\mathbf{a}, t)), \\
& \frac{\delta F}{\delta \pi_{i}}=\int_{\mathcal{D}_{\mathbf{x}}} d^{3} x \frac{\delta \bar{F}}{\delta M_{i}} \delta(\mathbf{x}-\mathbf{q}(\mathbf{a}, t))=\frac{\delta F}{\delta M_{i}^{\prime}}
\end{aligned}
$$

for $i=1,2,3$. In Eq. (90), the prime on $M_{i}^{\prime}$, indicates that $M_{i}^{\prime}$ depends on the parameter a. 
Making use of the expressions (89) and (90) into Eq. (81) one obtains

$$
\begin{aligned}
& \{F, G\}=\int_{\mathcal{D}_{\mathbf{x}}} d^{3} a\left(\frac{\delta F}{\delta q_{i}} \frac{\delta G}{\delta \pi_{i}}-\frac{\delta F}{\delta \pi_{i}} \frac{\delta G}{\delta q_{i}}\right) \\
& =\int_{\mathcal{D}_{\mathbf{x}}} d^{3} a\left[\int d^{3} x\left(\rho_{0} \frac{\partial}{\partial x_{i}} \frac{\delta \bar{F}}{\delta \rho}+\pi_{m} \frac{\partial}{\partial x_{i}} \frac{\delta \bar{F}}{\delta M_{m}}\right) \frac{\delta \bar{G}^{\prime}}{\delta M_{i}^{\prime}} \delta(\mathbf{x}-\mathbf{q}(\mathbf{a}, t))\right. \\
& \left.-\int_{\mathcal{D}_{\mathbf{x}}} d^{3} x\left(\rho_{0} \frac{\partial}{\partial x_{i}} \frac{\delta \bar{G}}{\delta \rho}+\pi_{m} \frac{\partial}{\partial x_{i}} \frac{\delta \bar{G}}{\delta M_{m}}\right) \frac{\delta \bar{F}}{\delta M_{i}^{\prime}} \delta(\mathbf{x}-\mathbf{q}(\mathbf{a}, t))\right] \\
& =-\int_{\mathcal{D}_{\mathbf{x}}} d^{3} x\left(\rho\left(\bar{F}_{\mathbf{M}} \cdot \nabla \bar{G}_{\rho}-\bar{G}_{\mathbf{M}} \cdot \nabla \bar{F}_{\rho}\right)+\mathbf{M} \cdot\left(\bar{F}_{\mathbf{M}} \cdot \nabla \bar{G}_{\mathbf{M}}-\bar{G}_{\mathbf{M}} \cdot \nabla \bar{F}_{\mathbf{M}}\right)\right) .
\end{aligned}
$$

where use was made of integration by part. Removing the overbars from the functionals in Eq. (91), one obtains namely the noncanonical Poisson bracket (48) for a barotropic fluid.

The transformation from Lagrangian to Eulerian variables is an example of reduction, a procedure which permits to obtain, from a Hamiltonian system, another Hamiltonian system with a smaller number of variables, by using symmetries of the initial system.

The case of the barotropic fluid treated above, in particular, falls in the category of reduction on the dual of a semidirect product Lie algebra $[51,52,57]$. The symmetry group consists of a group of diffeomorphisms on $\mathcal{D}_{\mathbf{x}}$, depending also on the parameter $\rho_{0}(\mathbf{a})$. The invariance of the Hamiltonian in Lagrangian coordinates with respect to such group, leads to the existence of a Hamiltonian reduced system, corresponding to that in Eulerian variables. The noncanonical reduced system (48) is defined on $\mathfrak{s}^{*}$, corresponding to the dual of the Lie algebra of the semidirect product group $S=\operatorname{Diff}\left(\mathcal{D}_{\mathbf{x}}\right)(S) \Upsilon\left(\mathcal{D}_{\mathbf{x}}\right)$, where we indicated with $\operatorname{Diff}\left(\mathcal{D}_{\mathbf{x}}\right)$ and $\Upsilon\left(\mathcal{D}_{\mathbf{x}}\right)$, the group of diffeomorphisms on $\mathcal{D}_{\mathbf{x}}$ and the space of functions on $\mathcal{D}_{\mathbf{x}}$, respectively.

The above derivation of the noncanonical Hamiltonian structure of a fluid model in Eulerian variables, from an action principle in terms of Lagrangian variables, can be extended to the case of various fluid plasma models. In particular, recent developments include the derivation of noncanonical Hamiltonian structures for inertial MHD [55], gyroviscous MHD $[58,59]$, extended MHD [54, 60] and relativistic MHD [61]. In spite of such progress, however, action principle derivations for the vast majority of Hamiltonian fluid models for plasmas are still lacking. In particular, this gap concerns most of the gyrofluid and drift-fluid models 
treated in Secs. IV and V.

Alternatively, a canonical Hamiltonian description, in Eulerian variables, can be obtained "inflating" the space of dynamical field variables by introducing Clebsch potentials [38]. Examples of such an approach to plasma fluid models include reduced MHD [62], ideal and Hall MHD [63, 64], where in the latter reference, a Lagrangian approach in terms of Clebsch variables is proposed.

\section{MHD MODELS WITH HAMILTONIAN CLOSURES}

The MHD description, according to which plasma is treated as a single conducting fluid, is very frequently adopted for modelling large scale phenomena of laboratory and astrophysical interest. In this Section, we review various closures that lead to non-dissipative MHD models possessing a Hamiltonian structure.

In order to simplify the notation, we will not indicate the domain of the integrals and the arguments of the functional derivatives. Because the Section will consider only fluid models, by the notation of Sec. II, we have $M=0$ and the domain is $\mathcal{D}_{\mathbf{x}}=[0,1] \times[0,1] \times[0,1]$ with periodic boundary conditions.

\section{A. Adiabatic MHD}

A fundamental breakthrough concerning the Hamiltonian formulation of continuum plasma models came with the results presented in Refs. [49, 56]. In these references, it was shown that the equations of ideal MHD for a plasma following an adiabatic equation of state, admit a Hamiltonian formulation. Such equations, in standard normalized Alfvén 
units, are given by

$$
\begin{aligned}
& \frac{\partial \rho}{\partial t}+\nabla \cdot(\rho \mathbf{v})=0 \\
& \frac{\partial \mathbf{v}}{\partial t}+(\mathbf{v} \cdot \nabla) \mathbf{v}+\frac{1}{\rho} \nabla p-\frac{1}{\rho}(\nabla \times \mathbf{B}) \times \mathbf{B}=0 \\
& \frac{\partial \mathbf{B}}{\partial t}-\nabla \times(\mathbf{v} \times \mathbf{B})=0 \\
& \frac{\partial s}{\partial t}+\mathbf{v} \cdot \nabla s=0
\end{aligned}
$$

where $\rho$ is the plasma mass density, $\mathbf{v}$ the velocity field, $\mathbf{B}$ the magnetic field and $s$ the entropy per unit mass. The pressure $p$ is still related to the internal energy by Eq. (42). However, here it is assumed that the internal energy depends on the entropy as well, so that $U=U(\rho, s)$. In particular, a "local" temperature $T$ of the plasma could be defined by the relation $T=\partial U / \partial s$. The adiabatic assumption, by definition, neglects the heat flow. Clearly, the following discussion includes the barotropic closure as a particular case, which is retrieved by assuming $U=U(\rho)$.

In addition to the continuity and velocity equations (92) and (93), the system includes also the induction equation (94) and the evolution equation for the entropy (95). Note that the entropy is simply advected by the flow. This is a consequence of having neglected the dissipation in the model and of having assumed an adiabatic closure, so that no heat can be exchanged between different portions of plasma. In the Lagrangian description, this corresponds to assuming the entropy per unit mass be a function of the particles labels [38].

Following Ref. [56], we recall that, in terms of the set of field variables $(\rho, \mathbf{v}, \mathbf{B}, s)$, the system (92)-(95) admits a Hamiltonian formulation with Hamiltonian functional

$$
H(\rho, \mathbf{v}, \mathbf{B}, s)=\int d^{3} x\left(\frac{\rho}{2} v^{2}+\rho U+\frac{B^{2}}{2}\right),
$$

and Poisson bracket

$$
\begin{aligned}
& \{F, G\}=-\int d^{3} x\left(F_{\rho} \nabla \cdot G_{\mathbf{v}}-G_{\rho} \nabla \cdot F_{\mathbf{v}}+\frac{\nabla \times \mathbf{v}}{\rho} \cdot G_{\mathbf{v}} \times F_{\mathbf{v}}\right. \\
& +\frac{\nabla s}{\rho} \cdot\left(F_{s} G_{\mathbf{v}}-F_{\mathbf{v}} G_{s}\right)+\frac{1}{\rho}\left(F_{\mathbf{v}} \cdot\left[\nabla G_{\mathbf{B}}\right]-G_{\mathbf{v}} \cdot\left[\nabla F_{\mathbf{B}}\right]\right) \cdot \mathbf{B} \\
& \left.+\mathbf{B} \cdot\left(\left[\nabla\left(\frac{F_{\mathbf{v}}}{\rho}\right)\right] \cdot G_{\mathbf{B}}-\left[\nabla\left(\frac{G_{\mathbf{v}}}{\rho}\right)\right] \cdot F_{\mathbf{B}}\right)\right),
\end{aligned}
$$


where $\mathbf{a} \cdot[\mathbf{M}] \cdot \mathbf{b} \equiv a_{i} M_{i j} b_{j}$ (with sum over repeated indices understood), for two vector $\mathbf{a}, \mathbf{b}$ and a matrix $\mathbf{M}$.

Assuming $\nabla \cdot \mathbf{B}=0$, the Hamiltonian (96) and the Poisson bracket (97) yield namely Eqs. (92)-(95).

We remark that the Hamiltonian (96) has the same form of the Hamiltonian (40), but with the additional contribution of the last term, corresponding to the magnetic energy. Also, the Poisson bracket (97) can be seen as the sum of the Poisson bracket (41), with the terms corresponding to the second and third line of Eq. (97), which introduce the evolution of the magnetic field and of the entropy.

The Poisson bracket (97) admits the following family of functionals

$$
C=\int d^{3} x \rho f(s)
$$

as Casimir invariants. In Eq. (98), $f$ is an arbitrary function. Among such functionals we recognize the total mass $\int d^{3} x \rho$ and the total entropy $\int d^{3} x \rho s$. Casimir invariants for the Poisson bracket of adiabatic MHD in terms of the magnetic vector potential are discussed in Refs. [41, 65, 66].

The extension from the MHD to the multi-fluid Hamiltonian description of plasmas following adiabatic equations of state was carried out in Ref. [67].

A formulation of the Poisson bracket for adiabatic MHD in terms of the magnetic vector potential was given in Ref. [68].

\section{B. Incompressible MHD : Dirac bracket}

A further closure, which is commonly adopted in the MHD description, corresponds to incompressibility, i.e. assuming that the velocity vector field satisfies $\nabla \cdot \mathbf{v}=0$. This is, for instance, appropriate [69], for slow phenomena, if the characteristic plasma velocity is much lower than the characteristic sound speed or Alfvén speed, depending on whether the plasma has $\beta \gg 1$ or $\beta \ll 1$, respectively, where we indicated with $\beta$ the ratio between the characteristic internal pressure and magnetic pressure. In particular, we will consider 
the case in which not only $\mathbf{v}$ is divergence-free but also the mass density is uniform and constant.

A procedure for imposing the incompressibility closure on Hamiltonian adiabatic MHD, so as to preserve the Hamiltonian character of the model, making use of Dirac's theory of constrained Hamiltonian systems was presented in Ref. [70]. The general theory of constrained Hamiltonian systems formulated by Dirac is described in References such as [71-74], and a previous application to fluid models for plasmas can be found in Ref. [75]. Here we simply sketch the main ideas about the procedure and we refer to the aforementioned publications for complete descriptions of the theory.

As mentioned above, Dirac's theory applies when it is needed to enforce constraints into a Hamiltonian system, in such a way that the constrained system remain Hamiltonian. Essentially, Dirac's theory provides a procedure to build a constrained Hamiltonian system possessing, as Hamiltonian, the Hamiltonian of the unconstrained system, but a new Poisson bracket (which we refer to as Dirac bracket) which accounts for the imposed constraints. In the context of a system of partial differential equations with field variables depending on a coordinate $\mathbf{x}$, such as Eqs. (92)-(95), one can consider imposing $K$ local constraints $\phi_{\alpha}(\mathbf{x})=0$, with $\alpha=1, \cdots, K$ ( global constraints, which are independent of $\mathbf{x}$, could be imposed as well but are less pertinent to the present scope ). Such constraints, in general, contain operators acting on the field variables.

The Dirac bracket is constructed from a Poisson bracket $\{$,$\} , first by determining the$ matrix $C$, whose elements are defined by

$$
C_{\alpha \beta}\left(\mathbf{x}, \mathbf{x}^{\prime}\right)=\left\{\phi_{\alpha}(\mathbf{x}), \phi_{\beta}\left(\mathbf{x}^{\prime}\right)\right\}
$$

Note that $C_{\alpha \beta}\left(\mathbf{x}, \mathbf{x}^{\prime}\right)=-C_{\beta \alpha}\left(\mathbf{x}^{\prime}, \mathbf{x}\right)$. Subsequently, one determines (if they exist) the inverse elements $C_{\alpha \beta}^{-1}\left(\mathbf{x}, \mathbf{x}^{\prime}\right)$ defined from the relations

$$
\int d^{3} x^{\prime} C_{\alpha \beta}^{-1}\left(\mathbf{x}, \mathbf{x}^{\prime}\right) C_{\beta \gamma}\left(\mathbf{x}^{\prime}, \mathbf{x}^{\prime \prime}\right)=\delta_{\alpha \gamma} \delta\left(\mathbf{x}-\mathbf{x}^{\prime \prime}\right)
$$

so that $C_{\alpha \beta}^{-1}\left(\mathbf{x}, \mathbf{x}^{\prime}\right)=-C_{\beta \alpha}^{-1}\left(\mathbf{x}^{\prime}, \mathbf{x}\right)$. 
If all the elements $C_{\alpha \beta}^{-1}\left(\mathbf{x}, \mathbf{x}^{\prime}\right)$ exist, so that the matrix $C$ is invertible in the sense described above, then the Dirac bracket $\{,\}_{*}$ is defined by

$$
\{F, G\}_{*}=\{F, G\}-\int d^{3} x \int d^{3} x^{\prime}\left\{F, \phi_{\alpha}(\mathbf{x})\right\} C_{\alpha \beta}^{-1}\left(\mathbf{x}, \mathbf{x}^{\prime}\right)\left\{\phi_{\beta}\left(\mathbf{x}^{\prime}\right), G\right\}
$$

It can be shown that the expression in Eq. (101) satisfies all the four properties of a Poisson bracket.

Moreover, making use of Eq. (100), it is relatively easy to see that the local functionals $\phi_{\alpha}(\mathbf{x})$ are Casimir invariants for the Dirac bracket. Therefore, the constraints $\phi_{\alpha}=0$, for $\alpha=1, \cdots, K$ are all automatically satisfied by a dynamics generated with the Dirac bracket (101). Denoting by $H\left(\chi_{1}, \cdots, \chi_{N}\right)$ the Hamiltonian functional of the unconstrained system, the Hamiltonian constrained equations of motion will be generated by the Dirac bracket (101) and the Hamiltonian functional $H$, and they will read

$$
\begin{aligned}
& \frac{\partial \chi_{i}(\mathbf{x})}{\partial t} \\
& =\left\{\chi_{i}(\mathbf{x}), H\right\}-\int d^{3} x^{\prime} \int d^{3} x^{\prime \prime}\left\{\chi_{i}(\mathbf{x}), \phi_{\alpha}\left(\mathbf{x}^{\prime}\right)\right\} C_{\alpha \beta}^{-1}\left(\mathbf{x}^{\prime}, \mathbf{x}^{\prime \prime}\right)\left\{\phi_{\beta}\left(\mathbf{x}^{\prime \prime}\right), H\right\}, \quad i=1, \cdots, N .
\end{aligned}
$$

In Eq. (102) we found it useful to emphasize the specific local dependence of the field variables and of the functions $\phi_{\alpha}$.

The first term on the right-hand side of Eq. (102) corresponds to the term generating the dynamics of the unconstrained system, whereas the second term is the additional contributions accounting for the constraints.

In case the $K$ constraints do not yield an invertible matrix $C$, then further secondary constraints have to be added. Such secondary constraints can be found from the condition that the primary $K$ constraints be preserved by the dynamics.

Considering now the specific case of incompressible MHD, we impose as primary constraint $\phi_{1}(\mathbf{x})=\rho(\mathbf{x})-\rho_{0}=0$, where $\rho_{0}$ is a uniform constant. Using the bracket (97) it follows that $C_{11}\left(\mathbf{x}, \mathbf{x}^{\prime}\right)=0$, so that the corresponding matrix $C$ is not invertible and secondary constraints must be introduced. From the continuity equation it follows that the 
first constraint is preserved by the dynamics, i.e. $\partial_{t} \phi_{1}(\mathbf{x})=0$, if $\nabla \cdot \mathbf{v}(\mathbf{x})=0$. Consequently we set as secondary constraints $\phi_{2}(\mathbf{x})=\nabla \cdot \mathbf{v}(\mathbf{x})=0$ (actually, the constraint is that $\nabla \cdot \mathbf{v}$ be time-independent and $\nabla \cdot \mathbf{v}=0$ is the particular initial condition of interest for incompressibility). This leads to

$$
\begin{aligned}
& C_{11}\left(\mathbf{x}, \mathbf{x}^{\prime}\right)=0, \quad C_{12}\left(\mathbf{x}, \mathbf{x}^{\prime}\right)=\Delta \delta\left(\mathbf{x}-\mathbf{x}^{\prime}\right), \\
& C_{21}\left(\mathbf{x}, \mathbf{x}^{\prime}\right)=-\Delta \delta\left(\mathbf{x}-\mathbf{x}^{\prime}\right), \quad C_{22}\left(\mathbf{x}, \mathbf{x}^{\prime}\right)=\nabla \cdot\left(\rho^{-1}(\nabla \times \mathbf{v}) \times \nabla \delta\left(\mathbf{x}-\mathbf{x}^{\prime}\right)\right) .
\end{aligned}
$$

It is now possible to find the inverse elements, which read

$$
\begin{aligned}
& C_{11}^{-1}\left(\mathbf{x}, \mathbf{x}^{\prime}\right)=\Delta^{-1} \nabla \cdot\left(\rho^{-1}(\nabla \times \mathbf{v}) \times \nabla \Delta^{-1} \delta\left(\mathbf{x}-\mathbf{x}^{\prime}\right)\right), \quad C_{12}^{-1}\left(\mathbf{x}, \mathbf{x}^{\prime}\right)=-\Delta^{-1} \delta\left(\mathbf{x}-\mathbf{x}^{\prime}\right), \\
& C_{21}^{-1}\left(\mathbf{x}, \mathbf{x}^{\prime}\right)=\Delta^{-1} \delta\left(\mathbf{x}-\mathbf{x}^{\prime}\right), \quad C_{22}\left(\mathbf{x}, \mathbf{x}^{\prime}\right)=0 .
\end{aligned}
$$

Making use of the inverse elements (104), from Eq. (101) it is now possible to construct the Dirac bracket, which, after some rearrangements, can be formulated as

$$
\begin{aligned}
& \{F, G\}_{*}=\int d^{3} x\left(\rho^{-1}(\nabla \times \mathbf{v}) \cdot\left(\bar{F}_{\mathbf{v}} \times \bar{G}_{\mathbf{v}}\right)-\rho^{-1} \nabla s \cdot\left(F_{s} \bar{G}_{\mathbf{v}}-\bar{F}_{\mathbf{v}} G_{s}\right)\right. \\
& \left.-\left(\rho^{-1} \bar{F}_{\mathbf{v}} \cdot\left[\nabla G_{\mathbf{B}}\right]-\rho^{-1} \bar{G}_{\mathbf{v}} \cdot\left[\nabla F_{\mathbf{B}}\right]\right) \cdot \mathbf{B}-\mathbf{B} \cdot\left(\left[\nabla\left(\rho^{-1} \bar{F}_{\mathbf{v}}\right)\right] \cdot G_{\mathbf{B}}-\left[\nabla\left(\rho^{-1} \bar{G}_{\mathbf{v}}\right)\right] \cdot F_{\mathbf{B}}\right)\right) .
\end{aligned}
$$

In Eq. (105) we introduced the constrained functional derivatives $\bar{F}_{\mathbf{v}}$, defined as $\bar{F}_{\mathbf{v}}=\mathcal{P} \cdot F_{\mathbf{v}}$, where $\mathcal{P}$ is the projector on the space of divergence-free vector fields. Such projector acts on a generic vector field $\mathbf{a}$ as $\mathcal{P} \cdot \mathbf{a}=\mathbf{a}-\nabla \Delta^{-1} \nabla \cdot \mathbf{a}$. It is easy to verify that $\nabla \cdot(\mathcal{P} \cdot \mathbf{a})=0$ and that $\mathcal{P}^{2}=\mathcal{P}$.

By comparing Eq. (105) with Eq. (97) one realizes that the Dirac bracket for an incompressible plasma corresponds to the Poisson bracket for an adiabatic plasma, but with the functional derivatives replaced by the constrained functional derivatives. The possibility of expressing a Dirac bracket with the same Poisson form of the original system, but using constrained functional derivatives, was shown in Ref. [76].

The equations of motion resulting from the Dirac bracket (105) and the Hamiltonian (96) 
correspond to

$$
\begin{aligned}
\frac{\partial \mathbf{v}}{\partial t}+(\mathbf{v} \cdot \nabla) \mathbf{v}+\nabla p-(\nabla \times \mathbf{B}) \times \mathbf{B} & =0, \\
\frac{\partial \mathbf{B}}{\partial t}-\nabla \times(\mathbf{v} \times \mathbf{B}) & =0, \\
\frac{\partial s}{\partial t}+\mathbf{v} \cdot \nabla s & =0,
\end{aligned}
$$

where one clearly supposes that the initial conditions satisfy $\nabla \cdot \mathbf{v}=0$.

In Eq. (106) the pressure corresponds to

$$
p=-\frac{v^{2}}{2}-\Delta^{-1} \nabla \cdot((\nabla \times \mathbf{v}) \times \mathbf{v})+\Delta^{-1} \nabla \cdot((\nabla \times \mathbf{B}) \times \mathbf{B}) .
$$

Unlike the adiabatic case, in the incompressible case, the pressure does not follow from the evolutions of the density and of the internal energy, but evolves in such a way that the condition $\partial_{t} \nabla \cdot \mathbf{v}=0$ is satisfied at all times. We also remark that, because of the constraint $\rho-\rho_{0}=0$, the mass density is no longer a dynamical variable, so that the set of field variables for incompressible MHD reduces to $(\mathbf{v}, \mathbf{B}, s)$.

The family of Casimirs (98) of adiabatic MHD degenerates then into the family

$$
C=\int d^{3} x f(s)
$$

with $f$ arbitrary function. Also, the role of the internal energy term in the Hamiltonian (96) becomes superfluous, and the dynamics is generated by the kinetic and magnetic energy only.

\section{Double adiabatic closure}

For nearly collisionless plasmas in the presence of a strong magnetic field, anisotropy in the pressure tensor can become significant. An anisotropic extension of the adiabatic

closure of Sec. III A is provided by the well known double adiabatic closure presented by Chew, Goldberger and Low in Ref. [36]. The equations of the MHD model with double 
adiabatic closure can be written as

$$
\begin{aligned}
& \frac{\partial \rho}{\partial t}+\nabla \cdot(\rho \mathbf{v})=0 \\
& \frac{\partial \mathbf{v}}{\partial t}+(\mathbf{v} \cdot \nabla) \mathbf{v}+\frac{1}{\rho} \nabla \cdot \mathbf{p}-\frac{1}{\rho}(\nabla \times \mathbf{B}) \times \mathbf{B}=0, \\
& \frac{\partial \mathbf{B}}{\partial t}-\nabla \times(\mathbf{v} \times \mathbf{B})=0, \\
& \frac{\partial s}{\partial t}+\mathbf{v} \cdot \nabla s=0 .
\end{aligned}
$$

Equations (111)-(114) coincide with Eqs. (92)-(95), apart from the closure term. Indeed, the gradient of the scalar pressure of Eq. (93) is replaced by the divergence of the pressure tensor $\mathbf{p}$ in Eq. (112). The pressure tensor is defined as

$$
\mathbf{p}=p_{\perp} \mathbf{I}+\frac{p_{\|}-p_{\perp}}{B^{2}} \mathbf{B B}
$$

where $p_{\perp}$ and $p_{\|}$indicate the pressures perpendicular and parallel to the magnetic field, respectively. Supposing that two relations

$$
f_{\perp}\left(p_{\perp} /(\rho B), s\right)=0, \quad f_{\|}\left(p_{\|} B^{2} / \rho^{3}, s\right)=0
$$

exist, for two functions $f_{\perp}$ and $f_{\|}$, then the perpendicular and parallel pressures obey the evolution equations

$$
\frac{d}{d t}\left(\frac{p_{\perp}}{\rho B}\right)=0, \quad \frac{d}{d t}\left(\frac{p_{\|} B^{2}}{\rho^{3}}\right)=0,
$$

where $d / d t=\partial_{t}+\mathbf{v} \cdot \nabla$ is the time derivative in a frame moving with the bulk plasma velocity.

The MHD system with double adiabatic closure is also amenable to a Hamiltonian formulation, which was provided in Ref. [56]. Eqs. (111)-(114) can indeed be generated from the same Poisson bracket (97) of adiabatic MHD and from the Hamiltonian (96) but upon extending the dependence of the internal energy $U$ to the amplitude of the magnetic field, so that $U=U(\rho, s, B)$. Introducing the relations

$$
p_{\perp}=\rho^{2} \frac{\partial U}{\partial \rho}+\rho B \frac{\partial U}{\partial B}, \quad p_{\|}=\rho^{2} \frac{\partial U}{\partial \rho},
$$


one obtains namely the MHD system with the double adiabatic closure. Because adiabatic and double adiabatic MHD share the same Poisson bracket, they also share the same Casimir invariants. We remark that a Hamiltonian relativistic extension of the double adiabatic MHD system was presented in Ref. [77].

\section{Barotropic extended MHD}

The usual MHD description is valid on scales $L \gg d_{i} \gg d_{e}$, where $d_{i}$ and $d_{e}$ correspond to the ion and electron skin depths, respectively. The fluid description of phenomena such as turbulence and magnetic reconnection can, however, often require the investigation of smaller scales. Then more refined versions of MHD become desirable. In Ref. [78], a model, which we denote as extended MHD, was presented. This model, like MHD, still assumes quasi-neutrality, but it accounts also for terms depending on ion and electron inertia, the remnants of the two-fluid description from which MHD is derived. Extended MHD provides a generalization of MHD, that applies also to characteristic scales $L \sim d_{e}$. Although the derivation of extended MHD dates back to more than half a century ago, the investigation of its conservation laws and Hamiltonian structure in the non-dissipative limit, is recent. Energy conservation properties for extended MHD were indeed described in Ref. [79] and its Hamiltonian formulation was presented in Ref. [80]. The extended MHD model treated

in Ref. [80] assumes a barotropic closure, thus showing that the Hamiltonian character of barotropic MHD survives an extension accouting for electron and ion scales. In normalized Alfvén units, with lenghts normalized by $L$, the barotropic extended MHD model reads

$$
\begin{aligned}
& \frac{\partial \rho}{\partial t}+\nabla \cdot(\rho \mathbf{v})=0 \\
& \frac{\partial \mathbf{v}}{\partial t}+(\mathbf{v} \cdot \nabla) \mathbf{v}+\frac{1}{\rho} \nabla p-\frac{1}{\rho}(\nabla \times \mathbf{B}) \times \mathbf{B}^{*}+d_{e}^{2} \nabla\left(\frac{(\nabla \times \mathbf{B})^{2}}{2 \rho^{2}}\right)=0 \\
& \frac{\partial \mathbf{B}^{*}}{\partial t}-\nabla \times\left(\mathbf{v} \times \mathbf{B}^{*}\right)+d_{i} \nabla \times\left(\frac{\nabla \times \mathbf{B}}{\rho} \times \mathbf{B}^{*}\right)-d_{e}^{2} \nabla \times\left(\frac{\nabla \times \mathbf{B}}{\rho} \times \nabla \times \mathbf{v}\right)=0
\end{aligned}
$$

where

$$
\mathbf{B}^{*}=\mathbf{B}+d_{e}^{2} \nabla \times\left(\frac{\nabla \times \mathbf{B}}{\rho}\right) .
$$


In Eq. (121) one can find the contributions due to ion and electron inertia, associated with the constants $d_{i}$ and $d_{e}$, respectively. These lead to a generalized Ohm's law, for which the magnetic flux is no longer frozen into the plasma. The contributions associated with $d_{e}$ in the velocity equations guarantee energy conservation. In the limit $d_{e}=d_{i}=0$ we recover the barotropic limit of Eqs. (92)-(95) with $p=p(\rho)$. In the limit $d_{e}=0$, on the other hand, one retrieves barotropic Hall MHD.

The Hamiltonian structure of Eqs. (119)-(121) can be formulated adopting $\left(\rho, \mathbf{v}, \mathbf{B}^{*}\right)$ as set of dynamical field variables. The Hamiltonian functional is given by

$$
H\left(\rho, \mathbf{v}, \mathbf{B}^{*}\right)=\int d^{3} x\left(\frac{\rho}{2} v^{2}+\rho U+\frac{B^{2}}{2}+d_{e}^{2} \frac{(\nabla \times \mathbf{B})^{2}}{2 \rho}\right),
$$

where $U=U(\rho)$ is the internal energy and where $\mathbf{B}$ has to be intended as the result of a symmetric operator acting on $\mathbf{B}^{*}$. In particular, one has $H_{\mathbf{B}^{*}}=\mathbf{B}$.

The Poisson bracket for barotropic extended MHD, on the other hand, reads

$$
\begin{aligned}
& \{F, G\}=-\int d^{3} x\left(F_{\rho} \nabla \cdot G_{\mathbf{v}}-G_{\rho} \nabla \cdot F_{\mathbf{v}}+\frac{\nabla \times \mathbf{v}}{\rho} \cdot G_{\mathbf{v}} \times F_{\mathbf{v}}\right. \\
& -\left(\frac{\mathbf{B}^{*}}{\rho} \cdot\left(F_{\mathbf{v}} \times\left(\nabla \times G_{\mathbf{B}^{*}}\right)\right)+\frac{\mathbf{B}^{*}}{\rho} \cdot\left(\left(\nabla \times F_{\mathbf{B}^{*}}\right) \times G_{\mathbf{v}}\right)\right) \\
& +d_{i}\left(\frac{\mathbf{B}^{*}}{\rho} \cdot\left(\left(\nabla \times F_{\mathbf{B}^{*}}\right) \times\left(\nabla \times G_{\mathbf{B}^{*}}\right)\right)\right) \\
& \left.-d_{e}^{2}\left(\frac{\nabla \times \mathbf{v}}{\rho} \cdot\left(\left(\nabla \times F_{\mathbf{B}^{*}}\right) \times\left(\nabla \times G_{\mathbf{B}^{*}}\right)\right)\right)\right) .
\end{aligned}
$$

In the limit $d_{e}=d_{i}=0$, the Hamiltonian (123) and the Poisson bracket (124) reduce to those of Ref. [49] for ideal MHD in the barotropic limit. In the limit $d_{e}=0$, on the other hand, one retrieves the Hamiltonian structure of Hall MHD [64, 81, 82]. An action principle derivation of the extended MHD Poisson bracket was provided in Refs. [54, 60].

If one is allowed to introduce an extended vector potential $\mathbf{A}^{*}$, such that $\mathbf{B}^{*}=\nabla \times \mathbf{A}^{*}$, the Poisson bracket (124) admits three independent Casimir invariants, which can be written as

$$
C_{1}=\int d^{3} x \rho, \quad C_{ \pm}=\int d^{3} x \mathbf{P}_{ \pm}^{*} \cdot\left(\nabla \times \mathbf{P}_{ \pm}^{*}\right)
$$

In Eq. (125), the vector fields $\mathbf{P}_{ \pm}^{*}$ indicate generalized canonical helicities and are defined 
by

$$
\mathbf{P}_{ \pm}^{*}=\mathbf{v}+\lambda_{ \pm} \mathbf{A}^{*}
$$

where the constants $\lambda_{ \pm}$correspond to $\lambda_{ \pm}=\left(-d_{i} \pm \sqrt{d_{i}^{2}+4 d_{e}^{2}}\right) /\left(2 d_{e}^{2}\right)$. The topological meaning of the invariants $C_{ \pm}$was investigated in Ref. [83].

The Hamiltonian formulation of the relativistic versions of extended MHD was recently presented in Ref. [61]. In Ref. [84], on the other hand, it was shown that the two-dimensional incompressible reduction of extended MHD still preserves a Hamiltonian structure. Here, the two-fluid nature of the corresponding Casimir invariants was described in the small mass ratio limit $d_{e}^{2} / d_{i}^{2} \ll 1$. On the other hand, as pointed out in Ref. [80], taking $d_{e} \rightarrow 0$ (i.e. taking the limit from extended to Hall MHD) or setting $d_{e}=0$ and taking $d_{i} \rightarrow 0$ (i.e. taking the limit from Hall MHD to ideal MHD), leads to singularities in the Casimir invariants $C_{ \pm}$.

With regard to the limit toward ideal MHD, we recall that it implies the removal of a singular perturbation, associated with the ion skin depth $d_{i}$. Indeed, as pointed out in Ref. [85], the removal of two-fluid effects which occurs in ideal MHD, leads to a qualitative change in the system. This reflects, for instance, in the equilibrium equations for the 2D incompressible case which, in ideal MHD, exhibit the Alfvénic singularity when $\mathbf{v}= \pm \mathbf{B}$. This corresponds to a change in the character of the equations from elliptic to hyperbolic. Hall MHD, on the other hand, admits two branches of equilibrium solutions, one of which, existing only for $d_{i} \neq 0$, has no singularities. This allows for a smooth transition from subAlfvénic to super-Alfvénic flows. Also, assuming a decomposition of the equilibrium fields in the form

$$
\mathbf{v}=\hat{z} \times \nabla \phi(x, y)+v_{z}(x, y) \hat{z}, \quad \mathbf{B}=\nabla \psi(x, y) \times \hat{z}+b_{z}(x, y) \hat{z}
$$

it turns out that, for ideal MHD, at equilibrium one has

$$
\phi=G(\psi)
$$

where $G$ is an arbitrary function. This implies that the perpendicular velocity is collinear with the perpendicular magnetic field at equilibrium. With regard to Hall MHD, one of the 
two branches of equilibrium solutions exhibits the same feature. On the other hand, for the regular branch, the equilibrium flow must satisfy

$$
\phi=Y(\psi)-d_{i} b_{z}
$$

where $Y$ is an arbitrary function. The presence of the Hall term allows then also for flows and magnetic fields not collinear in the perpendicular plane.

This difference between ideal and Hall MHD in the equilibrium configuration reflects the difference in the corresponding Casimir invariants. Indeed, equilibrium equations for noncanonical Hamiltonian systems can be obtained by extremizing [38, 41] the functional $H+\sum_{i} C_{i}$, with $H$ indicating the Hamiltonian and $C_{i}$ the Casimir invariants of the corresponding system. Equilibrium equations such as (128) and (129) depend then on the Casimir invariants of the system. In Ref. [85] is it shown how such equilibrium relations emerge also from integration of the hyperbolic equations of the equilibrium system. Finally, we remark that interesting relations between the Poisson brackets for extended MHD, Hall MHD and inertial MHD (which corresponds to the extended MHD system with $d_{i}=0$ ) were presented in Ref. [86].

\section{LOW FREQUENCY REDUCED FLUID MODELS WITH QUADRATIC NON- LINEARITIES AND STRONG GUIDE FIELD ASSUMPTION}

Although fluid models such as MHD or two-fluid plasma equations offer simplifications with respect to the original Vlasov-Maxwell system, they still consist of sets of nonlinear equations evolving a relatively high number of field variables. Further simplifications for specific applications are then desirable. Motivated mainly by the need to describe the lowfrequency dynamics of tokamak plasmas, a number of reduced fluid models were derived in the last decades. These models evolve $N$ fluid field variables $\left(\chi_{1}, \cdots, \chi_{N}\right)$ with $N$ typically less than the number of field variables involved in ordinary fluid models (note, for instance, that $N=8$ for adiabatic MHD in Sec. III A). These reduced models also assume the

presence of a magnetic field $\mathbf{B}=\mathbf{B}_{0}+\tilde{\mathbf{B}}$, with $\left|\mathbf{B}_{0}\right| \gg|\tilde{\mathbf{B}}|$, where $\mathbf{B}_{0}$ is a time-independent 
component and $\tilde{\mathbf{B}}$ is a time-dependent perturbation. This assumption serves to model the presence of a strong guide field in one direction, as it occurs for instance with the toroidal component of the magnetic field in tokamaks. Variations along the direction of the guide field are assumed to be much weaker than the variations in the plane perpendicular to $\mathbf{B}_{0}$. In general, the field variables will correspond to time-dependent fluctuations of physical fields. Their amplitude will be supposed to be small with respect to a time-independent equilibrium value. Time variations are also supposed to be slow, with respect to a characteristic time scale. In this Section we will consider for simplicity reduced models formulated in a slab geometry with a Cartesian coordinate system and assume that the strong guide field is in the $z$ direction. Assuming that the field variables $\chi_{1}, \cdots, \chi_{N}$ are normalized, and that time and distances are also normalized with respect to characteristic time and length scales, the above assumptions, characterizing the reduced field models treated in this Section, can be formulated more quantitatively in the following way:

$$
\begin{aligned}
& \partial_{t} \sim \partial_{z} \sim \chi_{1} \sim \chi_{2} \sim \cdots \sim \chi_{N} \sim \epsilon \ll 1 \\
& \partial_{x} \sim \partial_{y} \sim 1
\end{aligned}
$$

All the reduced models considered in this article retain only quadratic nonlinearities, that is terms of order $\epsilon^{2}$, on the basis of the ordering (130) (we remark, however, that the derivation of the low- $\beta$ reduced MHD model treated in Sec. IV A 1 does not require all the assumptions of Eq. (130)).

The term reduced associated with the fluid models treated in this Section refers then to a number of simplifying assumptions (low frequency, weak nonlinearities and weak gradients along the guide field direction). On the other hand, although reduced in this sense, such models, as will be seen in this Section, are amenable to several extensions, which make them a valuable and relatively simple tool for implementing, for instance, finite Larmor radius effects, higher order closures and gyrofluid descriptions.

Because we are still dealing with purely fluid models, we will consider the case $M=0$ and, in general, the same spatial domains of Sec. III, although, in some cases, when referring to the two-dimensional geometry, we could consider, as domain of the spatial coordinates of 
the fields, $\mathcal{D}_{\mathbf{x}}=[0,1] \times[0,1]$ with periodic boundary conditions $[87]$.

With regard to the Hamiltonian structure, all such reduced models share a common form of Hamiltonian functional and Poisson bracket, in the appropriate set of field variables. In particular, Hamiltonian functionals are quadratic forms

$$
H\left(\chi_{1}, \cdots, \chi_{N}\right)=<\chi \mid \mathcal{H} \chi>=\sum_{i, j=1}^{N} \int d^{3} x \chi_{i} \mathcal{H}^{i j} \chi_{j},
$$

where $\mathcal{H}$ is a linear, typically symmetric operator, with elements $\mathcal{H}^{i j}$, with $i, j=1, \cdots, N$.

The Poisson brackets, on the other hand, are of the general form

$$
\{F, G\}=\sum_{i, j, k=1}^{N} W_{k}^{i j} \int d^{3} x \chi_{k}\left[F_{\chi_{i}}, G_{\chi_{j}}\right]+\sum_{i, j=1}^{N} A^{i j} \int d^{3} x F_{\chi_{i}} \frac{G_{\chi_{j}}}{\partial z},
$$

where $W_{k}^{i j}$ and $A^{i j}$ are constants, and where we introduced the canonical bracket

$$
[f, g]=\frac{\partial f}{\partial x} \frac{\partial g}{\partial y}-\frac{\partial g}{\partial x} \frac{\partial f}{\partial y},
$$

for two functions $f$ and $g$. Poisson brackets of the form (132) can be built by extending Lie-Poisson brackets built on the dual of a Lie algebra [88]. In particular, the set of smooth functions on $\mathcal{D}_{\mathbf{x}}$, equipped with the Lie bracket (133), forms a Lie algebra that can be used as starting block to build Poisson brackets of the form (132). Not all the operations of the form (132) are Poisson brackets, though. Indeed, whereas bilinearity and the Leibniz identity are automatically satisfied, antisymmetry and the Jacobi identity impose restrictions on the coefficients $W_{k}^{i j}$ and $A^{i j}$. The results presented in Refs. [88, 89] provide, however, a practical procedure to verify if bilinear operations of the form (132) are Poisson brackets. Such procedure begins with considering the form (132) with $A^{i j}=0$ for all $i$ and $j$, therefore considering only the first term on the right-hand side of Eq. (132). Restricting to a twodimensional (2D) domain, this term can serve to build the Poisson bracket for the twodimensional reduction of the model, where the derivatives along the guide field (i.e. along z) are totally neglected. In this limit, Ref. [88] shows that antisymmetry is verified if and only if $W_{k}^{i j}=W_{k}^{j i}$ for all $i, j, k$. Moreover, it also shows that the Jacobi identity is satisfied if and only if the $N$ matrices $W^{(j)}$, defined by

$$
\left[W^{(j)}\right]_{k}^{i}=W_{k}^{i j}, \quad j=1, \cdots, N,
$$


pairwise commute. Once it is verified that set of coefficients $W_{k}^{i j}$ satisfy these properties, antisymmetry of the total bracket (132) is guaranteed if $A^{i j}=A^{j i}$, for all $i$ and $j$, and the Jacobi identity is verified if the coefficients $W_{k}^{i j}$ and $A^{i j}$ satisfy the relations

$$
A^{r s} W_{r}^{i j}=A^{r j} W_{r}^{s i}=A^{r i} W_{r}^{j s}
$$

where the sum over the repeated index $r$ is understood.

This suggests an effective procedure for identifying a Hamiltonian structure for a given reduced fluid model, one that was actually often adopted for the reduced models presented in this Section. Indeed, if a conserved functional of the form (131) is found for a given model, this can be used as candidate Hamiltonian. Combining this functional with a bracket of the form (132), one can see if the model equations can be obtained from the expression (10). If this is the case, then the above described results of Refs. [88, 89] can be used to check that antisymmetry and the Jacobi identity are satisfied, or to constrain the choice of

the coefficients $W_{k}^{i j}$ and $A^{i j}$ (there could be different sets of coefficients yielding the same equations of motion using the same Hamiltonian and, among these one should find the set for which the Jacobi identity is satisfied).

Different closures have been adopted in order to obtain reduced models and, due to the relative simplicity of such models, a number of sophisticated closure relations were tested on reduced models. In the next part of the Section we review reduced models with different closure schemes, for which the existence of a Hamiltonian structure has been proved.

\section{A. Drift-fluid models}

In this part of the Section we treat reduced models that, contrary to finite-Larmor-radius and gyrofluid models, neglect electron and ion finite Larmor radius effects. Such "drift-fluid models" can be formally derived from plasma two-fluid equations by means of so-called drift approximation $[6,30,31,90]$. 


\section{Low- $\beta$ reduced $M H D$}

Although originally derived by means of an asymptotic expansion from MHD [26], we find it is more reasonable to include low- $\beta$ reduced MHD in the category of drift-fluid models, in the sense that its equations can be formally obtained as a limit of fluid models derived from the drift approximation. This model represents a paradigmatic and relatively simple example of Hamiltonian reduced fluid model, in the sense described above.

In a simplified slab geometry, the low- $\beta$ reduced MHD equations read

$$
\begin{aligned}
& \frac{\partial \psi}{\partial t}+[\phi, \psi]+\frac{\partial \phi}{\partial z}=0, \\
& \frac{\partial \omega}{\partial t}+[\phi, \omega]+\nabla_{\|} \Delta_{\perp} \psi=0,
\end{aligned}
$$

where the operators $\Delta_{\perp}$ and $\nabla_{\|}$are defined by

$$
\Delta_{\perp} f=\frac{\partial^{2} f}{\partial x^{2}}+\frac{\partial^{2} f}{\partial y^{2}}, \quad \nabla_{\|} f=-[\psi, f]+\frac{\partial f}{\partial z}
$$

for a function $f$. In Eqs. (136)-(137), the scalar fields $\psi(\mathbf{x}, t)$ and $\phi(\mathbf{x}, t)$ correspond to the magnetic flux function and to the stream function, and are related to the magnetic and velocity fields by the relations $\mathbf{B}=\nabla \psi \times \hat{z}+B_{0} \hat{z}$ and $\mathbf{v}=\hat{z} \times \nabla \phi$, respectively, where $B_{0}$ is the amplitude of the guide field, which is uniform and constant, and $\hat{z}$ is the unit vector along the $z$ direction (analogous notation will be used for unit vectors along $x$ and $y$ ). The field $\omega=\hat{z} \cdot \nabla \times \mathbf{v}=\Delta_{\perp} \phi$, on the other hand, corresponds to the vorticity. From Ampère's law it also follows that $\Delta_{\perp} \psi$ is related to the parallel current density $\mathbf{J}$ by the relation $\mathbf{J}=-\Delta_{\perp} \psi \hat{z}$. At the level of approximation at which the low- $\beta$ reduced MHD equations are valid, the closure corresponds to that for a planar incompressible fluid. The simplifications introduced by the reduction are, however, evident if one compares Eqs. (136)-(137) with the incompressible full MHD equations (106)-(108).

The Hamiltonian formulation of Eqs. (136)-(137) dates back to Ref. [62]. Adopting $(\psi, \omega)$ as set of field variables, the Hamiltonian of the model is given by

$$
H(\psi, \omega)=\frac{1}{2} \int d^{3} x\left(\left|\nabla_{\perp} \phi\right|^{2}+\left|\nabla_{\perp} \psi\right|^{2}\right)
$$


where we introduced the perpendicualr gradient operator $\nabla_{\perp}$ defined by $\nabla_{\perp} f=\partial_{x} f \hat{x}+\partial_{y} f \hat{y}$. Making use of an integration by parts it can be seen that the Hamiltonian (139) has the form (131). The form (139), on the other hand, is more perspicuous from the physical point of view, for it shows that the total conserved energy is given by the sum of the kinetic and magnetic energies, corresponding to the first and second term of (139), respectively (the contribution of the uniform and constant guide field component is irrelevant for the expression of the total energy).

The Poisson bracket, on the other hand, reads

$$
\{F, G\}=\int d^{3} x\left(\omega\left[F_{\omega}, G_{\omega}\right]+\psi\left(\left[F_{\psi}, G_{\omega}\right]+\left[F_{\omega}, G_{\psi}\right]\right)+F_{\omega} \frac{\partial G_{\psi}}{\partial z}+F_{\psi} \frac{\partial G_{\omega}}{\partial z}\right)
$$

and is clearly of the form (132).

Upon integrating by parts and making use of the symmetry of the operator $\Delta_{\perp}$, with the prescribed boundary conditions, one obtains that $H_{\omega}=-\phi$ and $H_{\psi}=-\Delta_{\perp} \psi$. One can then verify that the Hamiltonian (139) and the Poisson bracket (140) yield namely Eqs. (136)-(137)

Casimir invariants of the Poisson bracket (140) are given by

$$
C_{1}=\int d^{3} x \psi, \quad C_{2}=\int d^{3} x \omega
$$

corresponding to total magnetic flux and vorticity, respectively. From the point of view of the Casimir invariants a more interesting situation occurs in the two-dimensional limit. Indeed, if one restricts to a domain described by coordinates $\mathbf{x}=(x, y) \in \mathcal{D}_{\mathbf{x}} \subseteq \mathbb{R}^{2}$, so that, in practice, the terms in (140) involving partial derivatives along $z$ are absent, Casimir invariants evolve to two infinite families, given by

$$
C_{1}=\int d^{2} x \mathcal{C}(\psi), \quad C_{2}=\int d^{2} x \omega \mathcal{G}(\psi)
$$

where $\mathcal{C}$ and $\mathcal{G}$ are arbitrary functions. We remark that $C_{1}$ expresses magnetic flux conservation, wheres $C_{2}$ includes the cross-helicity as a particular case.

The emergence of infinite families of Casimir invariants when imposing translational 
symmetry along the $z$ direction is a feature common to all the reduced models treated in this Section.

\section{Isothermal two-field model with electron inertia}

Motivated by the investigation of magnetic reconnection driven by electron inertia, the following two-field reduced model was proposed [91-93]:

$$
\begin{aligned}
& \frac{\partial \psi_{e}}{\partial t}+\left[\phi, \psi_{e}\right]-\rho_{s}^{2}[\omega, \psi]+\frac{\partial}{\partial z}\left(\phi-\rho_{s}^{2} \omega\right)=0, \\
& \frac{\partial \omega}{\partial t}+[\phi, \omega]+\nabla_{\|} \Delta_{\perp} \psi=0 .
\end{aligned}
$$

In Eqs. (143)-(144), $\psi_{e}=\psi-d_{e}^{2} \Delta_{\perp} \psi$ corresponds to the electron canonical momentum, and $d_{e}$ and $\rho_{s}$ indicate the normalized electron skin depth and sonic Larmor radius, respectively. In dimensional form, these two constants correspond to $d_{e}=c\left(m_{e} /\left(4 \pi n_{0} e^{2}\right)\right)^{1 / 2}$ and $\rho_{s}=$ $c\left(T_{e} M /\left(e^{2} B_{0}^{2}\right)\right)^{1 / 2}$, where $c$ is the speed of light, $m_{e}$ and $M$ the electron and ion mass, respectively, $e$ the proton charge and $n_{0}$ and $T_{e}$ the uniform equilibrium particle density and electron temperature. It follows then that the terms associated with the coefficients $d_{e}^{2}$ and $\rho_{s}^{2}$ in Eqs. (143)-(144) are proportional to the electron mass and temperature, respectively. Comparing Eqs. (143)-(144) with Eqs. (136)-(137), one sees that the isothermal model with electron inertia can be formally seen as an extension of reduced MHD accounting for effects of electron inertia and temperature. The stream function $\phi$ in the model (143)-(144) can actually be identified with a normalized electrostatic potential and corresponds to the stream function of the $\mathbf{E} \times \mathbf{B}$ velocity. Both models are valid in the low- $\beta$ approximation, although one should not forget that, in spite of the formal correspondences between the two models, reduced MHD was obtained from the MHD description, whereas the isothermal model with electron inertia is actually a two-field reduction of a more general three-field model [30], derived from the two-fluid plasma description in the drift approximation. In particular, for

such two-field model, the $\beta$ parameter refers only to the electron internal pressure, for ions are assumed to be cold.

The closure of the model consists indeed in imposing isothermal electrons, cold ions and 
a proportionality between electron density and $\mathbf{E} \times \mathbf{B}$ vorticity fluctuations. In the cold electron limit $\rho_{s}=0$, one retrieves the so called inertial MHD [55, 94]. The terms associated with electron inertia, on the other hand, break the magnetic frozen-in condition and allow for magnetic reconnection. If such terms are neglected, the magnetic field is again frozen-in but in a velocity field given by the sum of the $\mathbf{E} \times \mathbf{B}$ and electron diamagnetic velocities.

The Hamiltonian structure of the model consists of the Hamiltonian functional

$$
H\left(\psi_{e}, \omega\right)=\frac{1}{2} \int d^{3} x\left(\left|\nabla_{\perp} \phi\right|^{2}+\left|\nabla_{\perp} \psi\right|^{2}+d_{e}^{2}\left|\Delta_{\perp} \psi\right|^{2}+\rho_{s}^{2} \omega^{2}\right)
$$

and of the Poisson bracket

$$
\{F, G\}=\int d^{3} x\left(\psi_{e}\left(\left[F_{\psi_{e}}, G_{\omega}\right]+\left[F_{\omega}, G_{\psi_{e}}\right]\right)+\omega\left(d_{e}^{2} \rho_{s}^{2}\left[F_{\psi_{e}}, G_{\psi_{e}}\right]+\left[F_{\omega}, G_{\omega}\right]\right)+F_{\omega} \frac{\partial G_{\psi_{e}}}{\partial z}+F_{\psi_{e}} \frac{\partial G_{\omega}}{\partial z}\right)
$$

In the expression for the Hamiltonian one can notice the additional contributions due to the parallel kinetic energy and to the internal energy, corresponding to the third and fourth terms of Eq. (145), respectively.

Concerning the Poisson bracket, $C_{1}=\int d^{3} x \psi_{e}$ and $C_{2}=\int d^{3} x \omega$ are Casimir invariants for (146). As above anticipated, in the two-dimensional limit, two infinite families of Casimir invariants emerge, corresponding to

$$
C_{1}=\int d^{2} x \mathcal{C}_{+}\left(G_{+}\right), \quad C_{2}=\int d^{2} x \mathcal{C}_{-}\left(G_{-}\right)
$$

where $\mathcal{C}_{ \pm}$are arbitrary functions and where we introduced the fields

$$
G_{ \pm}=\psi_{e} \pm d_{e} \rho_{s} \omega
$$

By making use of the functional chain rule described in Sec. II, one can see that the twodimensional version of the Poisson bracket (146), when expressed in terms of the fields $\left(G_{+}, G_{-}\right)$, instead of $\left(\psi_{e}, \omega\right)$, takes the simplified form

$$
\{F, G\}=2 d_{e} \rho_{s} \int d^{2} x\left(G_{+}\left[F_{G_{+}}, G_{G_{+}}\right]-G_{-}\left[F_{G_{-}}, G_{G_{-}}\right]\right) .
$$

A generic feature of the class of Hamiltonian reduced fluid models treated in this Section is the existence of linear combinations (in this case, the fields $G_{ \pm}$) of the original field variables, 
in terms of which the Poisson bracket reduces to its simplest form. We refer to such field as to normal fields. Normal fields are typically suggested, as in this case, by the expression of the Casimir invariants. An important feature of normal fields is that they also simplify the model equations, putting in evidence properties of the model otherwise difficult to perceive in the original formulation. In the specific case, the model equations (143)-(144), when written in terms of the normal fields in the $2 \mathrm{D}$ limit, read

$$
\begin{aligned}
& \frac{\partial G_{+}}{\partial t}+\left[\phi_{+}, G_{+}\right]=0 \\
& \frac{\partial G_{-}}{\partial t}+\left[\phi_{-}, G_{-}\right]=0
\end{aligned}
$$

where

$$
\phi_{ \pm}=\phi \pm \frac{\rho_{s}}{d_{e}} \psi
$$

The reformulation of the model in terms of normal fields shows that the model actually

expresses the advection of two Lagrangian invariants (the normal fields $G_{ \pm}$) by means of generalized incompressible velocity fields $\mathbf{v}_{ \pm}=\hat{z} \times \nabla \phi_{ \pm}$associated with the stream functions $\phi_{ \pm}$. Topological conservation laws then exist, which are alternative to that associated with the magnetic frozen-in condition and that is violated by electron inertia. More precisely, in this case, by virtue of Eqs. (150)-(151), the contour lines of the normal fields $G_{ \pm}$will not be allowed to reconnect during the evolution of the system. This argument was used in a number of numerical investigations [91, 92, 95-98] of magnetic reconnection, to explain the formation of small scale structures and the saturation of magnetic islands in a dissipationless process.

\section{Four-field model with electron inertia}

A Hamiltonian extension of the two-field model of Sec. IV A 2, accounting for a more refined closure and including parallel ion velocity is given by the following system, which we 
denote as the four-field model with electron inertia (FFEI) :

$$
\begin{aligned}
& \frac{\partial \psi_{e}}{\partial t}+\left[\phi, \psi_{e}\right]+d_{\beta} \nabla_{\|} Z+\frac{\partial \phi}{\partial z}=0, \\
& \frac{\partial \omega}{\partial t}+[\phi, \omega]+\nabla_{\|} \Delta_{\perp} \psi=0 \\
& \frac{\partial Z}{\partial t}+[\phi, Z]-c_{\beta} \nabla_{\|} v-d_{\beta} \nabla_{\|} \Delta_{\perp} \psi=0, \\
& \frac{\partial v}{\partial t}+[\phi, v]-c_{\beta} \nabla_{\|} Z=0 .
\end{aligned}
$$

The 2D version of this model was derived in Ref. [31] and its 3D extension in Ref. [89]. Two additional field variables appear, with respect to the two-field model (143)-(144). The field $v$ corresponds to the parallel bulk velocity. On the other hand, in order to understand the meaning of the field $Z$, one has to recall that, in this four-field model, the magnetic field is assumed to be of the form (up to terms of the order $\epsilon^{2}$ ) $\mathbf{B}=B_{0} \hat{z}+\nabla \psi \times \hat{z}+c_{\beta} Z \hat{z}$. Consequently $Z$ represents a perturbation of the magnetic field along the direction of the guide field. This perturbation is multiplied times a constant coefficient $c_{\beta}$, defined as $c_{\beta}=$ $\sqrt{\beta /(1+\beta)}$, where $\beta$ is associated with the equilibrium electron pressure. The second coefficient appearing in the system is $d_{\beta}=d_{i} c_{\beta}$.

With regard to the closure relation, FFEI still assumes cold ions as does the two-field model. On the other hand, it goes beyond the isothermal closure for the electron fluid. Indeed, in normalized units, the model assumes electron pressure is of the form $p=p_{0}+B_{0} p_{1}$, where $p_{0}$ is the constant uniform equilibrium pressure and $p_{1}=p_{1}(\mathbf{x}, t)$ is a time dependent pressure perturbation, supposed to satisfy an adiabatic equation of state. The pressure perturbation field is, however, eliminated, by imposing the pressure balance relation $p_{1} \simeq$ $-c_{\beta} Z$. We remark that, contrary to the two-field model, here the value of $\beta$ is not restricted to be much less than unity. On the other hand, for $\beta \ll 1$, one has $c_{\beta} \sim \sqrt{\beta}$ and $d_{\beta} \sim \rho_{s}$. The terms proportional to $c_{\beta}$ can then be neglected at the leading order. This leads to a decoupling of the parallel velocity equation (156). Also, the solution $Z=-\rho_{s} \omega$ can be taken for $Z$. The two remaining equations (153)-(154), with $Z$ replaced by $-\rho_{s} \omega$ correspond namely to Eqs. (143)-(144). This shows how the two-field isothermal model can be formally retrieved from the low- $\beta$ limit of FFEI. 
The closure adopted for FFEI still allows for a Hamiltonian formulation of the model. In the 2D limit this was found in Ref. [99], whereas the 3D extension was provided in Ref. [89].

The Hamiltonian functional is given by

$$
H\left(\psi_{e}, \omega, Z, v\right)=\frac{1}{2} \int d^{3} x\left(|\nabla \psi|^{2}+|\nabla \phi|^{2}+d_{e}^{2}\left(\Delta_{\perp} \psi\right)^{2}+v^{2}+Z^{2}\right)
$$

with the last two terms indicating the additional contributions due to the parallel velocity and to the parallel magnetic perturbations coupled with the pressure fluctuations.

For the 2D limit, we report the expression of the Poisson bracket of Ref. [99], which reads

$$
\{F, G\}=\int d^{2} x\left(\omega\left[F_{\xi}, G_{\xi}\right]_{\omega}+\psi_{e}\left[F_{\xi}, G_{\xi}\right]_{\psi_{e}}+Z\left[F_{\xi}, G_{\xi}\right]_{Z}+v\left[F_{\xi}, G_{\xi}\right]_{v}\right)
$$

where

$$
\begin{aligned}
& \quad\left[F_{\xi}, G_{\xi}\right]_{\omega}=\left[F_{\omega}, G_{\omega}\right] \\
& {\left[F_{\xi}, G_{\xi}\right]_{Z}=\left[F_{Z}, G_{\omega}\right]+\left[F_{\omega}, G_{Z}\right]-d_{\beta} d_{e}^{2}\left[F_{\psi_{e}}, G_{\psi_{e}}\right]} \\
& \quad+c_{\beta} d_{e}{ }^{2}\left(\left[F_{v}, G_{\psi_{e}}\right]+\left[F_{\psi_{e}}, G_{v}\right]\right)-\alpha\left[F_{Z}, G_{Z}\right]-c_{\beta} \gamma\left[F_{v}, G_{v}\right], \\
& \quad\left[F_{\xi}, G_{\xi}\right]_{\psi_{e}}=\left[F_{\psi_{e}}, G_{\omega}\right]+\left[F_{\omega}, G_{\psi_{e}}\right]-d_{\beta}\left(\left[F_{Z}, G_{\psi_{e}}\right]+\left[F_{\psi_{e}}, G_{Z}\right]\right) \\
& \quad+c_{\beta}\left(\left[F_{v}, G_{Z}\right]+\left[F_{Z}, G_{v}\right]\right), \\
& {\left[F_{\xi}, G_{\xi}\right]_{v}=\left[F_{v}, G_{\omega}\right]+\left[F_{\omega}, G_{v}\right]} \\
& \quad+c_{\beta} d_{e}^{2}\left(\left[F_{Z}, G_{\psi_{e}}\right]+\left[F_{\psi_{e}}, G_{Z}\right]\right)-c_{\beta} \gamma\left(\left[F_{v}, G_{Z}\right]+\left[F_{Z}, G_{v}\right]\right),
\end{aligned}
$$

with $\alpha=d_{\beta}+c_{\beta} d_{e}^{2} / d_{i}, \gamma=d_{e}^{2} / d_{i}$.

The corresponding 2D Casimir invariants are given by

$$
\begin{aligned}
C_{1} & =\int d^{2} x \zeta \mathcal{C}(D), \\
C_{2} & =\int d^{2} x \mathcal{K}(D), \\
C_{ \pm} & =\int d^{2} x \mathcal{G}_{ \pm}\left(T_{ \pm}\right),
\end{aligned}
$$


where

$$
\begin{aligned}
D & =\psi_{e}+d_{i} v \\
\zeta & =\omega+\frac{Z}{\alpha}, \\
T_{ \pm} & = \pm \frac{d_{i}}{2 c_{\beta} d^{3} d_{e}}\left(d_{i} D-d^{2} v \mp d d_{e} Z\right),
\end{aligned}
$$

with $d=\sqrt{d_{i}^{2}+d_{e}^{2}}$, whereas $\mathcal{C}, \mathcal{K}$ and $\mathcal{G}_{ \pm}$are arbitrary functions.

The 2D verson of FFEI, adopting as dynamical variables the normal fields, reads

$$
\begin{aligned}
\frac{\partial D}{\partial t}+[\phi, D] & =0 \\
\frac{\partial \zeta}{\partial t}+[\phi, \zeta]-\frac{1}{d^{2}}[D, \psi] & =0 \\
\frac{\partial T_{+}}{\partial t}+\left[\phi_{+}, T_{+}\right] & =0, \\
\frac{\partial T_{-}}{\partial t}+\left[\phi_{-}, T_{-}\right] & =0
\end{aligned}
$$

where $\varphi_{ \pm}=\phi \pm\left(c_{\beta} d / d_{e}\right) \psi$ are generalized stream functions specific to this four-field model. We remark that in this case, unlike the two-field model, not all the equations turn into advection equations. Equation (166) expresses the conservation of the ion parallel canonical momentum D. Equation (167) is not an advection equation and governs the evolution of the generalized vorticity $\zeta$. Equations (168)-(169), on the other hand, generalize the advection equations (150)-(151) to a larger range of values for $\beta$.

We take advantage of the normal fields $\zeta, D, T_{ \pm}$, suggested by the expressions for the Casimir invariants (160)-(162), in order to write the 3D Poisson bracket in the more compact form

$$
\begin{aligned}
\{F, G\}=\int & d^{3} x\left(\zeta\left[F_{\zeta}, G_{\zeta}\right]+D\left(\left[F_{D}, G_{\zeta}\right]+\left[F_{\zeta}, G_{D}\right]\right)+T_{-}\left[F_{T_{-}}, G_{T_{-}}\right]\right. \\
& +T_{+}\left[F_{T_{+}}, G_{T_{+}}\right]+F_{\zeta} \frac{\partial}{\partial z} G_{D}+F_{D} \frac{\partial}{\partial z} G_{\zeta} \\
& \left.+\frac{d_{i}^{2}}{2 c_{\beta} d^{3} d_{e}} F_{T_{+}} \frac{\partial}{\partial z} G_{T_{+}}-\frac{d_{i}^{2}}{2 c_{\beta} d^{3} d_{e}} F_{T_{-}} \frac{\partial}{\partial z} G_{T_{-}}\right) .
\end{aligned}
$$

Results of numerical simulations of magnetic reconnection based on the 2D limit of the model were presented in Ref. [89]. 

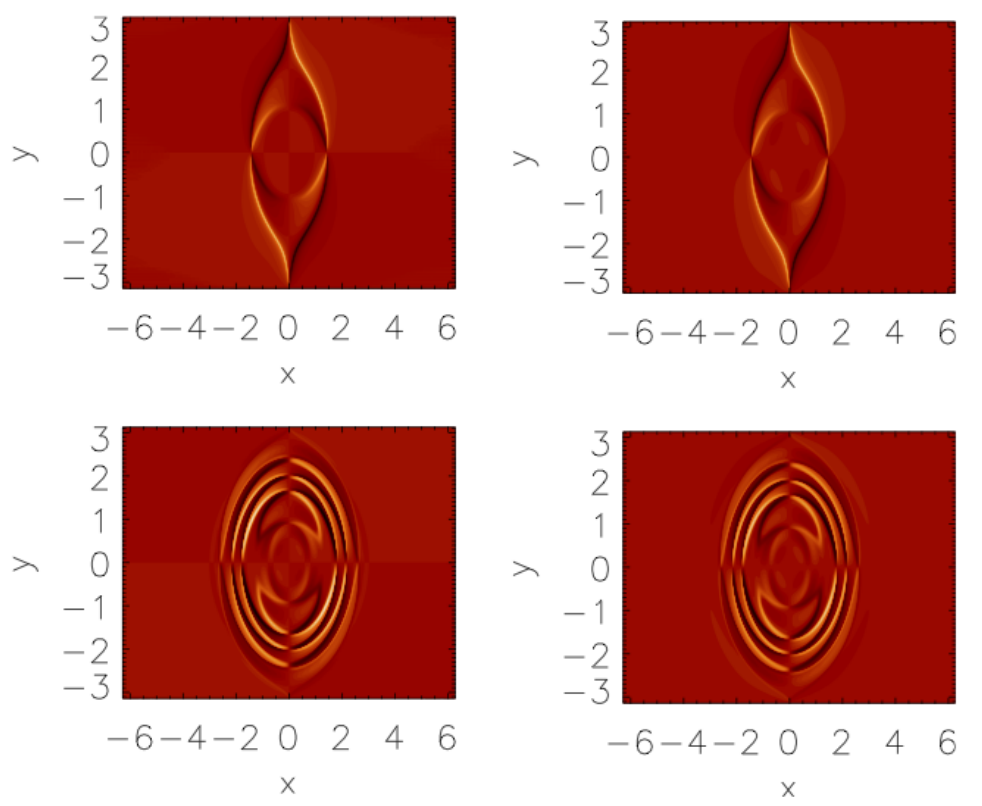

Figure 1. Contour plots of the fields $\omega$ (left column) and $Z$ (right column) at $t=50$ (top row) and $t=70$ (bottom row) Alfvén times. The plots refer to a low- $\beta$, large $d_{i}$ regime, with $c_{\beta}=$ $0.01, d_{\beta}=0.24, d_{e}=0.24$. In this regime, vorticity perturbations are proportional to parallel magnetic perturbations (after E. Tassi, P. J. Morrison, D. Grasso, F. Pegoraro, Nucl. Fusion, 50, $034007(2010))$.

From Fig. 1 it follows that, as expected, in the low- $\beta$ regime (but still with finite $d_{\beta}$, which means relatively large $d_{i}$ ), the dynamics of vorticity and parallel magnetic perturbations are similar and follow the behavior dictated by the isothermal two-field model. Flucutations begin with concentration around the magnetic island separatrices and, at later times, they tend to propagate inside the island. In a higher- $\beta$ regime with smaller $d_{i}$, on the other hand, the qualitative behavior of the $Z$ differs evidently from that of $\omega$, as shown in Fig. 2. The vorticity indeed exhibits very thin vertical jets aligned with the resonant line $x=0$ which propagate against each other and form mushroom-shaped structures centered at the $y=0$ line. Similar structures had been observed in previous simulations of the two-field model $[93,100]$ at low- $\beta$ but when $\rho_{s} \approx 0$. The four-field model then shows that such structures can manifest themselves not only at low- $\beta$ but also at larger $\beta$, provided that $d_{\beta}$ is finite. On the other hand, parallel magnetic perturbations tend to concentrate around the magnetic 

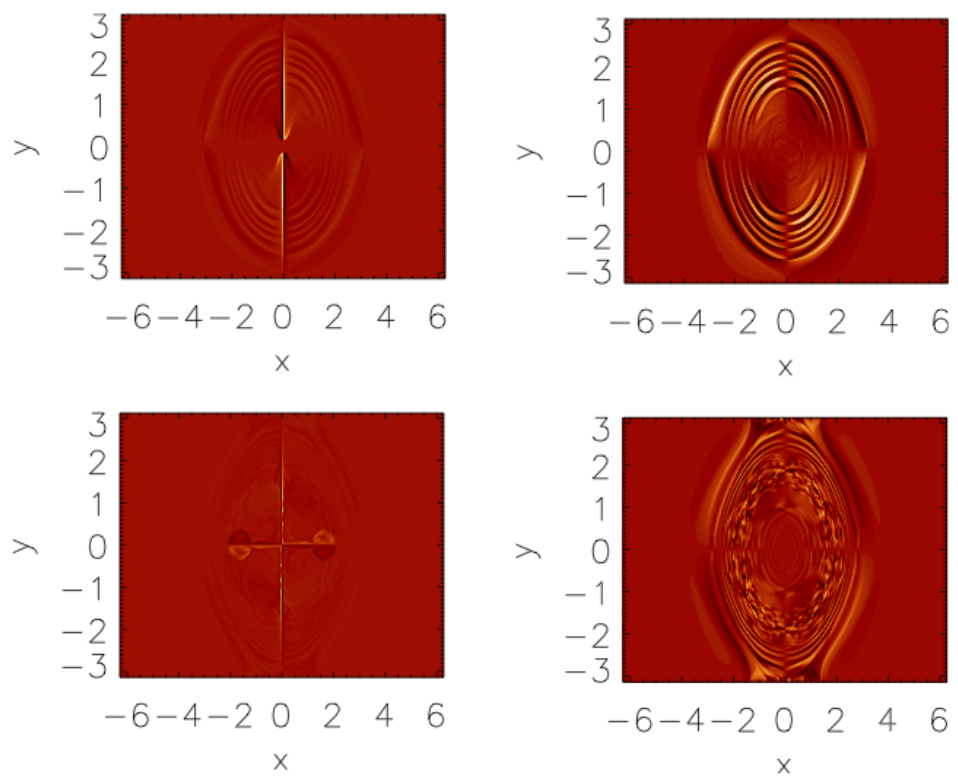

Figure 2. Contour plots of the fields $\omega$ (left column) and $Z$ (right column) at $t=40$ (top row) and $t=55$ (bottom row) Alfvén times. The plots refer to a higher- $\beta$ and lower $d_{i}$ regime, with respect to Fig. 1 . The values of the parameters are $c_{\beta}=0.3, d_{\beta}=0.72, d_{e}=0.24$. In this regime, vorticity perturbations detach from parallel magnetic perturbations (after E. Tassi, P. J. Morrison, D. Grasso, F. Pegoraro, Nucl. Fusion, 50, 034007 (2010)).

island separatrices and form small scale structures inside the island. Such differences in the dynamics of $\omega$ and $Z$ can be conveniently reinterpreted in terms of normal fields. Indeed, one has the relations

$$
Z=-\frac{c_{\beta} d^{2}}{d_{i}}\left(T_{+}+T_{-}\right), \quad \omega=\zeta+T_{+}+T_{-}
$$

The low- $\beta$ high $d_{i}$ regime corresponds to the case where $\zeta$, which is equal to zero at the initial condition, does not grow. Indeed, from Eq. (167), it follows that, for large $d_{i}$ (and therefore large $d$ ), the last term of the equation, which would allow $\zeta$ to grow, gives a negligible contribution. Therefore, if $\zeta \approx 0$, from Eq. (171) it follows that both $Z$ and $\omega$ are proportional to $T_{+}+T_{-}$. In the second regime, with higher $\beta$ and smaller $d_{i}, \zeta$ is allowed to grow. It is namely the generalized vorticity $\zeta$ which accounts for the thin stuctures observed in the contour plots of the vorticity. Depending on the region in the parameter space, different closures are then approached, leading to different qualitative behaviors in 
the vorticity and parallel magnetic perturbations.

\section{Four-field model with magnetic curvature}

We find it convenient to conclude the part of this Section devoted to Hamiltonian reduced drift-fluid models with a further four-field model which, although not recent, played an important role in the development of later Hamiltonian reduced models. In our context, it also serves as basis for useful remarks and comparisons with other models.

The model under consideration, which we refer to as four-field model with magnetic curvature (FFMC) is given by the following system:

$$
\begin{aligned}
& \frac{\partial \psi}{\partial t}+[\phi, \psi]-\delta \nabla_{\|} p+\frac{\partial \phi}{\partial z}=0, \\
& \frac{\partial \omega}{\partial t}+[\phi, \omega]+\nabla_{\|} \Delta_{\perp} \psi+[h, p]=0, \\
& \frac{\partial p}{\partial t}+[\phi, p]+\beta \nabla_{\|} v+2 \delta \beta \nabla_{\|} \Delta_{\perp} \psi-2 \beta[h, \phi-\delta p]=0, \\
& \frac{\partial v}{\partial t}+[\phi, v]+\frac{1}{2} \nabla_{\|} p=0 .
\end{aligned}
$$

In Eqs. (172)-(175) p indicates the normalized electron pressure fluctuations, $v$ the parallel ion velocity, whereas $h$ accounts for the curvature of the guide field at the lowest order, and the expression $h(x)=x$ can be taken, with $x$ mimicking the radial coordinate of toroidal geometry. The expression for the constant $\delta$ is given by $\delta=d_{i} / 2$. Although in this model an

evolution equation for the pressure appears, the pressure balance argument of the model of Sec. IV A 3 still applies, so that Eq. (174) can be replaced by an equation for the evolution of the parallel perturbation of the equilibrium magnetic field.

The model (172)-(175) is a cold-ion limit of the four-field model derived in Ref. [6]. Its Hamiltonian structure was given in Ref. [101]. After defining the field $\bar{p}=p+2 \beta h$, one can choose $(\psi, \omega, \bar{p}, v)$ as set of dynamical field variables. In terms of these, the Hamiltonian structure of the model consists of the Hamiltonian functional

$$
H(\psi, \omega, \bar{p}, v)=\frac{1}{2} \int d^{3} x\left(|\nabla \psi|^{2}+|\nabla \phi|^{2}+v^{2}+\frac{(\bar{p}-2 \beta h)^{2}}{2 \beta}\right)
$$


and of the Poisson bracket

$$
\begin{aligned}
& \{F, G\}=\int d^{3} x\left(\omega\left[F_{\omega}, G_{\omega}\right]\right. \\
& +\psi\left(\left[F_{\omega}, G_{\psi}\right]+\left[F_{\psi}, G_{\omega}\right]+2 \delta \beta\left(\left[F_{\psi}, G_{\bar{p}}\right]+\left[F_{\bar{p}}, G_{\psi}\right]\right)-\beta\left(\left[F_{\bar{p}}, G_{v}\right]+\left[F_{v}, G_{\bar{p}}\right]\right)\right) \\
& +\bar{p}\left(\left[F_{\omega}, G_{\bar{p}}\right]+\left[F_{\bar{p}}, G_{\omega}\right]+2 \delta \beta\left[F_{\bar{p}}, G_{\bar{p}}\right]\right) \\
& +v\left(\left[F_{\omega}, G_{v}\right]+\left[F_{v}, G_{\omega}\right]\right)+2 \delta \beta\left(F_{\psi} \frac{\partial G_{\bar{p}}}{\partial z}+F_{\bar{p}} \frac{\partial G_{\psi}}{\partial z}\right) \\
& \left.+F_{\omega} \frac{\partial G_{\psi}}{\partial z}+F_{\psi} \frac{\partial G_{\omega}}{\partial z}-\beta\left(F_{\bar{p}} \frac{\partial G_{v}}{\partial z}+F_{v} \frac{\partial G_{\bar{p}}}{\partial z}\right)\right) .
\end{aligned}
$$

Casimir invariants correspond to

$$
C_{1}=\int d^{3} x \zeta, \quad C_{2}=\int d^{3} x D, \quad C_{3}=\int d^{3} x \bar{p}, \quad C_{4}=\int d^{3} x \psi,
$$

where we define

$$
\zeta=\omega-\frac{\bar{p}}{2 \delta \beta}, \quad D=\psi+2 \delta v
$$

Note that in Eq. (179) we voluntarily used the same symbols for the fields adopted in Eqs. (163) and (164). Indeed, $\zeta$ and $D$ are analogous quantities in the two models, indicating the generalized vorticity and the parallel ion canonical momentum. In the 2D limit the Casimir invariants are given by the four infinite families

$$
\begin{aligned}
C_{1} & =\int d^{2} x \zeta \mathcal{C}(D), & C_{2} & =\int d^{2} x \mathcal{K}(D), \\
C_{3} & =\int d^{2} x \bar{p} \mathcal{S}(\psi), & C_{4} & =\int d^{2} x \mathcal{G}(\psi),
\end{aligned}
$$

and the normal fields $(D, \zeta, \bar{p}, \psi)$ evolve according to

$$
\begin{aligned}
& \frac{\partial D}{\partial t}+[\phi, D]=0 \\
& \frac{\partial \zeta}{\partial t}+[\phi, \zeta]-\frac{1}{4 \delta^{2}}[D, \psi]=0 \\
& \frac{\partial \bar{p}}{\partial t}+[\phi, \bar{p}]-\beta[D, v]-2 \delta \beta\left[\psi, \Delta_{\perp} \psi\right]+2 \beta \delta[h, \bar{p}]=0 \\
& \frac{\partial \psi}{\partial t}+[\bar{\phi}, \psi]=0
\end{aligned}
$$

where we introduced $\bar{\phi}=\phi-\delta p$, corresponding to the stream function of a velocity field given by the sum of the $\mathbf{E} \times \mathbf{B}$ and electron diamagnetic velocity. 
It is interesting to compare, in particular in the light of their Hamiltonian structures and conservation properties, the four-field model FFEI of Sec. IV A 3 with FFMC in the 2D limit. The model equations differ only by a few terms. In particular, if electron inertia corrections are removed from FFEI (by setting $d_{e}=0$ ), one formally obtains FFMC in the limit of straight and uniform equilibrium magnetic field (i.e. $h=0$ ), provided that in the latter one performs the replacements: $\beta \rightarrow 2 c_{\beta}^{2}, \delta \rightarrow d_{i} / 2, p \rightarrow-2 c_{\beta} Z$. In particular, the inclusion of terms associated with magnetic curvature, do not alter the form of the Poisson bracket and of the Hamiltonian, so that all the related considerations for the FFMC can be made, without loss of generality, by taking the limit $h=0$ and, consequently, $\bar{p}=p$. In spite of the apparently little differences introduced in the model equations by electron inertia, qualitative differences appear in terms of Casimir invariants and evolution of normal fields. Indeed, electron inertia introduces a significant number of additional terms in the Poisson bracket (158). As a consequence, the families of Casimir invariants $C_{3,4}$ differ qualitatively in the two models. In FFMC, the magnetic frozen-in condition holds, which is reflected in the existence of the Casimir invariants $C_{3,4}$. Indeed, $C_{4}$ expresses magnetic flux conservation. On the other hand, taking into account that pressure perturbations are proportional to parallel magnetic perturbations by virtue of the pressure balance, both $C_{3}$ and $C_{4}$ (choosing $\mathcal{S}(\psi)=\psi$ ) contribute to the conservation of the total magnetic helicity $\int d^{3} x \mathbf{A} \cdot \mathbf{B}$, where A is the magnetic vector potential. Inclusion of electron inertia breaks these conservation laws and, similarly to what occurred with the two-field model, replaces them with alternative topological conservation laws associated with the normal fields $T_{ \pm}$. Two advection equations, Eqs. (168) and (169) take the place of the advection equation (185) and of the evolution equation (184). A similar feature occurs when moving from low- $\beta$ reduced MHD to the twofield model of Sec. IV A 2. The appearance of the two advection equations (150)-(151) is indeed a consequence of the inclusion of electron inertia and finite electron temperature (this latter contribution, had already a counterpart in FFMC, represented by the third term in Eq. (172), so that, between the two four-field models, the qualitative change in the Casimir invariants was due to the addition of electron inertia only). We remark, however, that, an 
ordering which systematically neglects electron Larmor radius effects while retaining electron inertia, would imply a low $\beta$, and consequently, as above discussed, a three-field reduction of FFEI, with a decoupling of Eq. (156) [102].

We mention also that a relevant limit of FFMC consists of setting $\delta=0$, which yields the equations of compressible reduced MHD [6], the Hamiltonian structure of which was discussed in Refs. [88, 101, 103].

Further connections between the normal fields for drift-fluid reduced models and closures will be discussed in Sec. VB 2.

\section{B. Adding ion finite Larmor radius effects while preserving a Hamiltonian struc-} ture : the gyromap

The reduced fluid models treated in Sec. IV A all possess a Hamiltonian structure, but they neglect, by taking the zero-thermal Larmor radius limit, phenomena occurring on the thermal ion Larmor radius scale. Clearly, this imposes limitations on the range of applicability of such models. More general closures accounting for FLR effects would then be desirable. A procedure, now denoted as the gyromap, was first proposed in Ref. [104], in order to add ion FLR effects to cold-ion Hamiltonian models, in such a way that the resulting model still possess a Hamiltonian structure. In Ref. [58] the origin of the gyromap procedure from an action principle perspective is discussed. In Ref. [101], the gyromap was applied in order to build a Hamiltonian FLR version of the cold-ion model FFMC. We report the resulting model, with a slight change in the notation with respect to Ref. [101].

$$
\begin{aligned}
& \frac{\partial \psi}{\partial t}+[\phi, \psi]-\delta \nabla_{\|} p+\frac{\partial \phi}{\partial z}=0, \\
& \frac{\partial W}{\partial t}+[\varphi, W]+\nabla_{\|} \Delta_{\perp} \psi+(1+\tau)\left(1+\rho_{i}^{2} \Delta_{\perp}\right)[h, p] \\
& -\delta \tau\left(\nabla_{\perp} \cdot\left[p+2 \beta h, \nabla_{\perp} \varphi\right]+\frac{1}{2} \rho_{i}^{2} \Delta_{\perp}[p+2 \beta h, W]\right)+\rho_{i}^{2} \Delta_{\perp} \nabla_{\|}\left(\Delta_{\perp} \psi+\frac{v}{2 \delta}\right)=0, \\
& \frac{\partial p}{\partial t}+[\phi, p]+\beta \nabla_{\|} v+2 \delta \beta \nabla_{\|} \Delta_{\perp} \psi-2 \beta[h, \phi-\delta p]=0, \\
& \frac{\partial v}{\partial t}+[\phi, v]+\frac{1}{2} \nabla_{\|}(p+\tau(p-\delta \beta W))-\rho_{i}^{2}\left[v, \Delta_{\perp}(\varphi-\delta \tau p)\right]-\frac{2 \rho_{i}^{2}}{\delta}[v, h]=0 .
\end{aligned}
$$


In Eqs. (186)-(189), $\tau=T_{i} / T_{e}$ indicates the ratio between the uniform equilibrium temperatures of the ion and electron populations, whereas $\rho_{i}=\delta \sqrt{\tau \beta}=\sqrt{T_{i} / M} /\left(\omega_{c i} L\right)$ is the normalized ion thermal radius, with $\omega_{c i}$ indicating the ion cyclotron frequency referred to $B_{0}$. The field variable $W$ is defined by $W=\Delta_{\perp} \varphi$, where the generalized stream function $\varphi$ satisfies

$$
\left(1+\rho_{i}^{2} \Delta_{\perp}\right) \varphi=\phi+\delta \tau p
$$

In the cold-ion limit, corresponding to $\tau=\rho_{i}=0$, one retrieves FFMC, as expected. The Hamiltonian structure of Eqs. (186)-(189), obtained from the gyromap procedure, was provided in Ref. [101] and we report it here. The Hamiltonian functional reads

$$
H(W, \psi, p, v)=\frac{1}{2} \int d^{3} x\left(\left|\nabla_{\perp} \psi\right|^{2}+\left|\nabla_{\perp} \varphi\right|^{2}+v^{2}+(1+\tau) \frac{p^{2}}{2 \beta}\right) .
$$

Note that it possesses the same form of the Hamiltonian (176) of FFMC, with the additional contribution of the ion pressure and the replacement of the electrostatic potential with the generalized stream function. The Poisson bracket is given by

$$
\begin{aligned}
& \{F, G\}=\int d^{3} x\left(\xi\left(F_{\xi}, G_{\xi}\right]+2 \delta^{2} \tau \beta\left[F_{v}, G_{v}\right]\right) \\
& +\psi\left(\left[F_{\xi}, G_{\psi}\right]+\left[F_{\psi}, G_{\xi}\right]+2 \delta \beta\left(\left[F_{\psi}, G_{\bar{p}}\right]+\left[F_{\bar{p}}, G_{\psi}\right]\right)-\beta\left(\left[F_{\bar{p}}, G_{v}\right]+\left[F_{v}, G_{\bar{p}}\right]\right)\right) \\
& +\bar{p}\left(\left[F_{\xi}, G_{\bar{p}}\right]+\left[F_{\bar{p}}, G_{\xi}\right]+2 \delta \beta\left[F_{\bar{p}}, G_{\bar{p}}\right]-\delta \tau\left[F_{v}, G_{v}\right]\right) \\
& +v\left(\left[F_{\xi}, G_{v}\right]+\left[F_{v}, G_{\xi}\right]\right) \\
& \left.+F_{\xi} \frac{\partial G_{\psi}}{\partial z}+F_{\psi} \frac{\partial G_{\xi}}{\partial z}+2 \delta \beta\left(F_{\psi} \frac{\partial G_{\bar{p}}}{\partial z}+F_{\bar{p}} \frac{\partial G_{\psi}}{\partial z}\right)-\beta\left(F_{\bar{p}} \frac{\partial G_{v}}{\partial z}+F_{v} \frac{\partial G_{\bar{p}}}{\partial z}\right)\right),
\end{aligned}
$$

where we introduced the field variable $\xi=W-(\delta \tau / 2) \Delta_{\perp} p$ and where $\bar{p}$ is defined as in Sec. IV A 4. Casimir invariants in the 2D limit are given by

$$
\begin{array}{r}
C_{1}=\int d^{2} x \bar{p} \mathcal{C}(\psi), \quad C_{2}=\int d^{2} x \mathcal{K}(\psi), \\
C_{ \pm}=\int d^{2} x \mathcal{G}_{ \pm}\left(2 \delta \beta v+\beta \psi \pm \sqrt{2} \rho_{i}\left(2 \delta \beta W-\rho_{i}^{2} \Delta_{\perp} p-\bar{p}\right)\right),
\end{array}
$$

where $\mathcal{C}, \mathcal{K}$ and $\mathcal{G}_{ \pm}$are arbitrary functions. Note that the Casimir invariants (193) share the same structure of the Casimir invariants (180) of FFEI. The role of the parallel canonical ion momentum $D$ in the latter (where $D$ is a Lagrangian invariant due to the cold-ion 
hypothesis) is played in $C_{1}$ and $C_{2}$ of Eq. (193) by $\psi$, which is a Lagrangian invariant of the former model due to the assumption of neglecting electron inertia. Both models then possess families of invariants $C_{ \pm}$which are corresponding generalizations of the families (147) of the two-field isothermal model. The four 3D Casimir invariants of the bracket (192) correspond, as already seen with previous models, to integrals of the four normal fields that can be identified from Eqs. (193)-(194).

Recently [105], an FLR model for interchange instability, corresponding to a two-field electrostatic reduction of Eqs. (186)-(189), was derived in two alternative ways: by applying the gyromap procedure from a cold-ion model and by asymptotic expansion from the Braginskii equations [4]. This confirmed that all the FLR corrections introduced by the gyromap correspond to a consistent ordering. In order to illustrate the basic ideas underlying the gyromap procedure, we summarize its application in Ref. [105], because, although less general than the original application of Ref. [101], it possesses the advantage of being simpler, still containing the main ingredients.

The starting point is the cold-ion two-field model

$$
\begin{aligned}
& \frac{\partial \omega}{\partial t}+[\phi, \omega]+[h, n]=0, \\
& \frac{\partial n}{\partial t}+[\phi, n]+[\phi, h]-[n, h]=0,
\end{aligned}
$$

which could formally be obtained as a $2 \mathrm{D}$ reduction of the FFMC neglecting the evolutions of the magnetic field and of the parallel velocity, replacing the pressure perturbations in favor of the density perturbations $n$ and setting $\beta=1 / 2$ and $\delta=1$ (different normalizations are adopted in the two models, so that replacements in the values of the coefficients has only a formal utility). The model (195)-(196) can be obtained from the Hamiltonian

$$
H(\omega, n)=\frac{1}{2} \int d^{2} x\left(|\nabla \phi|^{2}+n^{2}\right)
$$

and the Poisson bracket

$$
\{F, G\}=\int d^{2} x\left((n+h)\left(\left[F_{n}, G_{n}\right]+\left[F_{n}, G_{\omega}\right]+\left[F_{\omega}, G_{n}\right]\right)+\omega\left[F_{\omega}, G_{\omega}\right]\right) .
$$


The gyromap provides a prescription to build new Hamiltonian and Poisson bracket, where the FLR model equations can be obtained from, so that the resulting FLR model is Hamiltonian by construction.

The FLR Hamiltonian is assumed to be given by

$$
H(W, n)=\frac{1}{2} \int d^{2} x\left((1+\tau) n^{2}+|\nabla \varphi|^{2}\right) .
$$

The Hamiltonian (199) is built form the cold-ion Hamiltonian (197) by adding the contribution (associated with $\tau$ ) due to the ion internal energy, and by replacing the kinetic energy term with the second term on the right-hand side of Eq. (199), which depends on a new stream function $\varphi$. A new field variable $W$ was also introduced, which is related to the new stream function by $W=\Delta_{\perp} \varphi$. The relation between the new variables and the old ones is left unspecified at the moment and will be retrieved a posteriori.

An auxiliary variable $\xi$ is now introduced and defined by $W=\xi+\tau \Delta_{\perp} n / 2$. The crucial point here is that the shift $\tau \Delta_{\perp} n / 2$ is half the first order of the normalized parallel component of the curl of the magnetization velocity $\nabla \times \Upsilon / n$, where we indicated $\Upsilon=-n T_{i} \hat{z} / B_{0}$ the magnetization [27]. This is namely the shift required in order to yield the FLR corrections.

One then writes the same Poisson bracket (198) of the cold-ion model, in terms of new field variables $(N, \xi)$ :

$$
\{F, G\}=\int d^{2} x\left((N+h)\left(\left[F_{N}, G_{N}\right]+\left[F_{N}, G_{\xi}\right]+\left[F_{\xi}, G_{N}\right]\right)+\xi\left[F_{\xi}, G_{\xi}\right]\right) .
$$

Considering the change of variables $(N, \xi) \rightarrow(n, W)$, one can, by making use of the functional chain rule, write the bracket (200) in terms of the variables $(n, W)$ :

$$
\begin{aligned}
& \{F, G\}=\int d^{2} x\left(( n + h ) \left(\left[F_{n}+(\tau / 2) \Delta_{\perp} F_{W}, G_{W}\right]+\left[F_{W}, G_{n}+(\tau / 2) \Delta_{\perp} G_{W}\right]\right.\right. \\
& \left.\left.+\left[F_{n}+(\tau / 2) \Delta_{\perp} F_{W}, G_{n}+(\tau / 2) \Delta_{\perp} G_{W}\right]\right)+\left(W-(\tau / 2) \Delta_{\perp} n\right)\left[F_{W}, G_{W}\right]\right) .
\end{aligned}
$$

From the Poisson bracket (201) and the Hamiltonian (199), we obtain the equations of motion for the FLR model. In particular, the continuity equation reads

$$
\frac{\partial n}{\partial t}+\left[\varphi+(\tau / 2) \Delta_{\perp} \varphi, n+h\right]-(1+\tau)[n, h]=0 .
$$


Because we require, on physical grounds, that the continuity equation should not be affected by leading order FLR corrections, we impose that the new continuity equation (202) coincide with its cold-ion counterpart (196). This provides the relation

$$
\left(1+\frac{\tau}{2} \Delta_{\perp}\right) \varphi=\phi+\tau n
$$

between the new stream function $\varphi$ and the variables $n$ and $\phi$.

The evolution equation for the FLR-corrected vorticity $W$, on the other hand, is given by

$$
\frac{\partial W}{\partial t}+[\varphi, W]-(1+\tau)\left(1+\frac{\tau}{2} \Delta_{\perp}\right)[n, h]-\tau \nabla_{\perp} \cdot\left[n+h, \nabla_{\perp} \varphi\right]+\frac{\tau^{2}}{4} \Delta_{\perp}[W, n+h]=0 .
$$

Using Eqs. (202), (203) and (204) one obtains, up to the normalization, a 2D electrostatic reduction of Eqs. (187)-(188). We remark that an important point in obtaining the Poisson bracket (201) is that it was obtained from the cold-ion bracket, which, by hypothesis, is a Poisson bracket. Consequently, the FLR -corrected bracket is also a Poisson bracket, which avoids, in particular, complicated verifications a posteriori of the Jacobi identity. We observe, however, that the application of the gyromap to obtain the entire four-field model (186)-(189) contained an additional complication due to the FLR-corrections for the dynamics parallel to the guide field, which implied a modification of the cold-ion bracket, before applying the mapping involving the magnetization velocity.

Finally, we remark that examples of Hamiltonian reduced fluid models including FLR effects are described also in Refs. [104, 106, 107].

\section{Gyrofluid models}

Although the gyromap offers a procedure to implement ion FLR corrections while preserving a Hamiltonian structure, such corrections are typically restricted to the limit $k_{\perp} \rho_{i} \ll 1$, where $k_{\perp}$ represents the characteristic wavenumber of the perturbations on the plane perpendicular to the equilibrium guide field. Modelling fluctuations on smaller scales, such that $k_{\perp} \rho_{i} \sim 1$, is, however, well known to be important for tokamak plasma turbulence 
(see, e.g. Ref. [108]). Such scales play an important role in determining the turbulent spectrum of astrophysical plasmas [109]. At the level of the kinetic description, gyrokinetics offers a valuable tool for investigating phenomena occurring at the ion gyroradius scale (see, e.g. Ref. [15]). The quest for more tractable, although less complete, models still valid at the ion gyroradius scale, motivated the derivation of fluid reductions of gyrokinetic models, denoted as gyrofluid models [16]. Contrary to ordinary fluid models, which are derived by considering moments of the particle distribution functions, gyrofluid models evolve moments of the gyrocenter distribution functions. Such distribution functions describe the states of populations of gyrocenters, whose coordinates evolve in a reduced phase space, obtained by asymptotically eliminating the dependence on the gyration angle from the original sixdimensional particle phase space. The evolution of the gyrocenter distribution functions is governed namely by the gyrokinetic equations.

With respect to drift-fluid or FLR models, such as those treated in Secs. IV A and IV B, gyrofluid models are valid in the regime $k_{\perp} \rho_{i} \sim 1$, at the expense of being formulated in terms of gyrocenter fluid moments, which, possibly, offer a less immediate physical interpretation, with respect to ordinary fluid moments. The transformation from gyrocenter to particle moments can be carried out explicitly, in some cases, as for instance in the $k_{\perp} \rho_{i} \ll 1$ limit $[16]$.

Highly nonlinear gyrofluid models were formulated also in recent years (see, e.g. Ref. [25]). For such models, however, no Hamiltonian structure is known, to the best of our knowledge. On the other hand, a number of Hamiltonian gyrofluid models were derived, in particular in the last decade, in the reduced framework consistent with the ordering (130).

In the following we discuss first a family of Hamiltonian gyrofluid models characterized by an isothermal closure on the electrons. Subsequently, we consider a recent Hamiltonian gyrofluid models accounting for parallel temperature and heat flux electron fluctuations. In all such models, only the ion population is described in terms of gyrofluid moments. For the electrons, gyroradius effects are neglected, assuming that $\rho_{e} \ll \rho_{i}$, with $\rho_{e}=\left(T_{e} / T_{i}\right)^{1 / 2}\left(m_{e} / M\right)^{1 / 2} \rho_{i}$ indicating the electron thermal gyroradius. Also, in all these 
models, a low- $\beta$ limit is assumed and parallel magnetic perturbations are neglected, i.e. $Z=0$.

In this Section, we restrict to the case of periodic boundary conditions on bounded domains, so that fields can be represented in Fourier series.

\section{Gyrofluid models with isothermal electrons}

Historically, a precursor of the family of existing Hamiltonian reduced gyrofluid models, can be found in Ref. [110]. It consists of a two-field model evolving the parallel electron canonical momentum and a vorticity-like field (proportional to the electron density fluctuations). Ion gyro-effects are included in the quasi-neutrality relation by making use of a Padé approximant for the gyroaverage operator. The electron fluid is supposed to be isothermal. In more recent years, progressive extensions of this model, with different closures for the ion fluid, were formulated, all possessing a Hamiltonian structure. In Ref. [34] a three-field model including also ion gyrocenter density fluctuations was proposed. In Ref. [111], a four-field model was presented, which accounts also for parallel ion dynamics and magnetic curvature. Finally, Ref. [33], describes a five-field model which includes also ion parallel temperature fluctuations. All such models can be derived as truncations of the electromagnetic gyrofluid model of Ref. [21]. Also, all these models assume an isothermal closure for the electron fluid. With regard to the closure on the gyroaverage operator for the ion fluid, the expression introduced in Ref. [19] was adopted in all models. Moreover, all such models, unlike the various drift-fluid models presented in Sec. IV A, possess similar Hamiltonian structures, in the sense that the corresponding Casimir invariants are associated with normal fields that obey advection equations in the 2D limit, and that the Hamiltonian functionals are built by adding quadratic energy contributions as the number of fields increases. Therefore, in order to avoid redundancies in the exposition, we describe explicitly only the most recent five-field model of Ref. [33], and indicate how the previous models can be obtained 
in particular limits. The five-field model equations that we are considering are given by

$$
\begin{aligned}
& \frac{\partial n_{e}}{\partial t}+\left[\phi, n_{e}\right]+\nabla_{\|} u_{e}-2 \kappa\left[h, \rho_{s}^{2} n_{e}-\phi\right]=0, \\
& \frac{\partial \psi_{e}}{\partial t}+\left[\phi, \psi_{e}\right]-\rho_{s}^{2} \nabla_{\|} n_{e}+2 \kappa d_{e}^{2} \rho_{s}^{2}\left[h, u_{e}\right]+\frac{\partial \phi}{\partial z}=0, \\
& \frac{\partial N_{i}}{\partial t}+\left[\Phi, N_{i}\right]+\bar{\nabla}_{\|} U_{i}+2 \kappa\left[h, \rho_{i}^{2} N_{i}+\Phi+\rho_{i}^{2} T_{\| i}\right]=0, \\
& \frac{\partial \Psi_{i}}{\partial t}+\left[\Phi, \Psi_{i}\right]+\rho_{i}^{2} \bar{\nabla}_{\|} N_{i}+\rho_{i}^{2} \bar{\nabla}_{\|} T_{\| i}+4 \kappa d_{i}^{2} \rho_{i}^{2}\left[h, U_{i}\right]+\frac{\partial \Phi}{\partial z}=0, \\
& \frac{\partial T_{\| i}}{\partial t}+\left[\Phi, T_{\| i}\right]+\bar{\nabla}_{\|} U_{i}+2 \kappa\left[h, \rho_{i}^{2} N_{i}+\Phi+\rho_{i}^{2} T_{\| i}\right]=0,
\end{aligned}
$$

and are complemented by the quasi-neutrality relation

$$
\frac{\Gamma_{0}-1}{\rho_{i}^{2}} \phi+\Gamma_{0}^{1 / 2} N_{i}-n_{e}=0
$$

and by Ampère's law

$$
\Delta_{\perp} \psi+\Gamma_{0}^{1 / 2} U_{i}-u_{e}=0
$$

In Eqs. (205)-(209) we introduced the field variables

$$
\psi_{e}=\psi-d_{e}^{2} u_{e}, \quad \Psi_{i}=\Psi+d_{i}^{2} U_{i}
$$

indicating the electron and ion parallel canonical momenta, respectively. Ion gyrofluid moments (in order to distinguish them from moments with respect to the particle distribution functions) are indicated with the uppercase symbols $N_{i}, U_{i}$ and $T_{\| i}$, corresponding to gyrocenter density, parallel velocity and parallel temperature fluctuations, respectively. The electron moments, on the other hand, are indicated with $n_{e}$ and $u_{e}$, corresponding to the density and parallel velocity fluctuations, respectively. The expression for the gyroaverage operator $\Gamma_{0}[19]$, in Fourier space, corresponds to multiplication times $I_{0}\left(k_{\perp}^{2} \rho_{i}^{2}\right) \exp \left(-k_{\perp}^{2} \rho_{i}^{2}\right)$, with $I_{0}$ indicating the modified Bessel function of order zero. We also introduced the symbols $\Phi, \Psi$ and $\bar{\nabla}_{\|}$, involving the gyroaverage operator, and whose definitions are as follows:

$$
\Phi=\Gamma_{0}^{1 / 2} \phi, \quad \Psi=\Gamma_{0}^{1 / 2} \psi, \quad \bar{\nabla}_{\|} f=-[\Psi, f]+\frac{\partial f}{\partial z}
$$

for a function $f$. In order to facilitate the comparison with other models discussed in this article, we modified the normalization and the notation, with respect to Ref. [33]. 
The coefficient $\kappa$, which accounts for the background magnetic curvature corresponds to $\kappa=u_{d} / d_{i}$, where $u_{d}$ is the term in the original notation, defined as $u_{d}=L_{n} / R$, with $L_{n}$ and $R$ being the density scale-length and the radius of curvature of the magnetic field, respectively. Also, apart form differences in the normalization and in the notation, the system (205)-(209) differs from Eqs. (3)-(7) of Ref. [33], for it corrects a few typographical errors present in the original reference.

The closure at the level of ion temperature fluctuations, makes the five-field model (205)(209) an effective tool for investigating ion temperature driven instabilitites, such as the ion temperature gradient instability. As noted above, it follows a series of Hamiltonian gyrofluid models with lower-order closures. Indeed, neglecting ion temperature fluctuations, one retrieves the four-field model of Ref. [111]. If, in addition, one neglects parallel ion dynamics and magnetic curvature, the three-field model of Ref. [34] is obtained. Finally, if also the ion gyrocenter density fluctuations are suppressed and a Padé approximant is taken for the gyroaverage operator $\Gamma_{0}$, one recovers the two-field model of Ref. [110]. With regard to this family of gyrofluid models, the following remark about the connection between closures and Hamiltonian structures was pointed out in Ref. [33]: whereas in the parallel ion momentum equation (208), the term associated with magnetic curvature has a coefficient 4 , in the isothermal version of Ref. [111], the corresponding coefficient is 2 . The reason for this difference resides in the constraint that the models be Hamiltonian. In particular, the Jacobi identity imposes the coefficients 4 and 2 in the five and four-field model, respectively. Modifications of the magnetic curvature terms (and possibly of other terms due to effects neglected in this model) must therefore be applied when varying the closure and requiring that the resulting model remain Hamiltonian.

Choosing $\left(n_{e}, \psi_{e}, N_{i}, \Psi_{i}, T_{\| i}\right)$ as dynamical variables, the Hamiltonian for the five-field model (205)-(209) can be written as

$H\left(n_{e}, \psi_{e}, N_{i}, \Psi_{i}, T_{\| i}\right)=\frac{1}{2} \int d^{3} x\left(\rho_{s}^{2} n_{e}^{2}+\rho_{i}^{2} N_{i}^{2}+d_{e}^{2} u_{e}^{2}+d_{i}^{2} U_{i}^{2}+\rho_{i}^{2} T_{\| i}^{2}+\left|\nabla_{\perp} \psi\right|^{2}+\Phi N_{i}-\phi n_{e}\right)$.

The first two terms and the fifth term represent energy contributions due to density and 
temperature fluctuations, the third and fourth term correspond to the parallel kinetic energies for electrons and ion gyrocenters, respectively, whereas the last three terms account for the magnetic and electrostatic energies.

The corresponding Poisson bracket can be written as

$$
\{F, G\}=\{F, G\}_{e}+\{F, G\}_{i}
$$

where

$$
\begin{aligned}
& \{F, G\}_{e}=\int d^{3} x\left(\tilde{n}_{e}\left(\left[F_{n_{e}}, G_{n_{e}}\right]+d_{e}^{2} \rho_{s}^{2}\left[F_{\psi_{e}}, G_{\psi_{e}}\right]\right)+\psi_{e}\left(\left[F_{\psi_{e}}, G_{n_{e}}\right]+\left[F_{n_{e}}, G_{\psi_{e}}\right]\right)\right) \\
& \left.+F_{n_{e}} \frac{\partial G_{\psi_{e}}}{\partial z}+F_{\psi_{e}} \frac{\partial G_{n_{e}}}{\partial z}\right) \\
& \{F, G\}_{i}=-\int d^{3} x\left(\tilde{N}_{i}\left(\left[F_{N_{i}}, G_{N_{i}}\right]+d_{i}^{2} \rho_{i}^{2}\left[F_{\Psi_{i}}, G_{\Psi_{i}}\right]+\left[F_{T_{\| i}}, G_{T_{\| i}}\right]\right)\right. \\
& +\Psi_{i}\left(\left[F_{\Psi_{i}}, G_{N_{i}}\right]+\left[F_{N_{i}}, G_{\Psi_{i}}\right]+\left[F_{\Psi_{i}}, G_{T_{\| i}}\right]+\left[F_{T_{\| i}}, G_{\Psi_{i}}\right]\right) \\
& +\tilde{T}_{\| i}\left(\left[F_{N_{i}}, G_{T_{\| i}}\right]+\left[F_{T_{\| i}}, G_{N_{i}}\right]+d_{i}^{2} \rho_{i}^{2}\left[F_{\Psi_{i}}, G_{\Psi_{i}}\right]\right) \\
& \left.+F_{\Psi_{i}} \frac{\partial G_{N_{i}}}{\partial z}+F_{N_{i}} \frac{\partial G_{\Psi_{i}}}{\partial z}+F_{T_{\| i}} \frac{\partial G_{\Psi_{i}}}{\partial z}+F_{\Psi_{i}} \frac{\partial G_{T_{\| i}}}{\partial z}\right)
\end{aligned}
$$

with

$$
\tilde{n}_{e}=n_{e}-2 \kappa x, \quad \tilde{N}_{i}=N_{i}-2 \kappa x, \quad \tilde{T}_{\| i}=T_{\| i}-2 \kappa x .
$$

We remark that the Poisson bracket $(215)$ is given by the sum of two contributions, $\{,\}_{e}$ and $\{,\}_{i}$ associated with electron and ion quantities, respectively. Each of these two contributions is of the form (132). Moreover, $\{,\}_{e}$ has a linear dependence only on the electron field variables $n_{e}, \psi_{e}$ and contains functional derivatives only with respect to such fields. The same occurs with $\{,\}_{i}$ but referred to the gyrofluid ion moments $N_{i}, \Psi_{i}, T_{\| i}$. The two contributions $\{,\}_{e}$ and $\{,\}_{i}$ can then be seen as two Poisson brackets with Poisson operators depending only on electron and ion fields, respectively, and also, with functional derivatives acting separately with respect to electron and ion fields. Consequently, by virtue of the Lemma of Ref. [56], mentioned in Sec. IIB, if $\{,\}_{e}$ and $\{,\}_{i}$ are Poisson brackets, their direct sum (215) is also a Poisson bracket. This shows that adopting the separated electron and ion moments as the set of field variables, offers a practical advantage for the 
verification of the Jacobi identity. Also, it simplifies the construction of Hamiltonian models with different closures for electrons and ions, for one needs only to check the Jacobi identity separately for the Poisson brackets involving the electron and ion fields. Given that the Poisson bracket is the direct sum of two Poisson brackets depending only on electron and ion fields, the coupling between electron and ion fields in the equations of motion is provided by the Hamiltonian. In particular, the electromagnetic energy (the last three terms in Eq. (214)), couple ion and electron quantities, via quasi-neutrality and Ampère's law.

In the 2D limit, the Poisson bracket (215) possesses the following five infinite families of Casimir invariants:

$$
\begin{array}{rlrl}
C_{e 1} & =\int d^{2} x \mathcal{C}_{e+}\left(G_{+}\right), & C_{e 2}=\int d^{2} x \mathcal{C}_{e-}\left(G_{-}\right), \\
C_{i 1} & =\int d^{2} x \mathcal{C}_{i+}\left(I_{+}\right), & C_{i 2}=\int d^{2} x \mathcal{C}_{i-}\left(I_{-}\right), \\
C_{i 3} & =\int d^{2} x \mathcal{C}_{i}(S) & &
\end{array}
$$

where $\mathcal{C}_{e \pm}, \mathcal{C}_{i \pm}$ and $\mathcal{C}_{i}$ are arbitrary functions and where we introduced the normal fields

$$
\begin{aligned}
& G_{ \pm}=\psi_{e} \pm d_{e} \rho_{s} \tilde{n}_{e}, \\
& I_{ \pm}=\sqrt{2} \Psi_{i} \pm d_{i} \rho_{i}\left(\tilde{N}_{i}+\tilde{T}_{\| i}\right), \\
& S=N_{i}-T_{\| i} .
\end{aligned}
$$

Clearly, the fields $G_{ \pm}$are reminiscent of the normal fields (148) (indeed, also in the fivefield model electrons are treated as an isothermal fluid). The fields $I_{ \pm}$represent their ion gyrocenter counterparts, whereas the field $S$ measures the departure from the adiabaticity condition for the ion fluid.

In terms of the normal fields, Eqs. (205)-(209) can be recast in the following form:

$$
\begin{aligned}
& \frac{\partial G_{ \pm}}{\partial t}+\left[\phi_{ \pm}, G_{ \pm}\right]+\frac{\partial \phi_{ \pm}}{\partial z}=0, \\
& \frac{\partial I_{ \pm}}{\partial t}+\left[\Phi_{ \pm}, G_{ \pm}\right]+\sqrt{2} \frac{\partial \Phi_{ \pm}}{\partial z}=0, \\
& \frac{\partial S}{\partial t}+[\Phi, S]=0
\end{aligned}
$$


where we introduced the generalized stream functions

$$
\begin{aligned}
& \phi_{ \pm}=\phi \pm d_{e} \rho_{s} u_{e}-\rho_{s}^{2} n_{e}, \\
& \Phi_{ \pm}=\Phi \pm \sqrt{2} d_{i} \rho_{i} U_{i}+\rho_{i}^{2}\left(N_{i}+T_{\| i}\right) .
\end{aligned}
$$

The apparent mismatch between the generalized stream functions (152) for the two-field model and those, given by (227), pertaining to the electron fluid in the five-field model, can be reconciled by noting that the replacement, in Eqs. (150)-(151) $\phi_{ \pm}$with alternative stream functions $\phi_{ \pm}^{\prime}=\phi_{ \pm} \mp\left(\rho_{s} / d_{e}\right) G_{ \pm}=\phi \pm d_{e} \rho_{s} \Delta_{\perp} \psi-\rho_{s}^{2} \omega$ does not modify Eqs. (150)-(151). The

stream functions $\phi_{ \pm}^{\prime}$, on the other hand, have a form analogous to (227). The form (152) can be more perspicuous from the physical point of view for, as will be seen in Sec. VB, it reflects the combination of the $\mathbf{E} \times \mathbf{B}$ velocity with the free motion along the magnetic field in the gyrocenter dynamics.

\section{2D gyrofluid model with parallel electron heat flux dynamics}

Motivated by the interest in going beyond the electron isothermal closure, for the investigation of magnetic reconnection driven by electron inertia, a 2D Hamiltonian six-field model accounting for isothermal gyrofluid ions and parallel electron heat flux dynamics was proposed in Ref. [35]. This model may be considered to lie, in terms of completeness and simplicity, between the isothermal two-field model of Sec. IV A 2 and hybrid reduced models for inertial reconnection, where electrons obey a drift-kinetic description [112, 113]. 
The model evolution equations are given by

$$
\begin{aligned}
& \frac{\partial n_{e}}{\partial t}+\left[\phi, n_{e}\right]-\left[\psi, u_{e}\right]=0, \\
& \frac{\partial \psi_{e}}{\partial t}+\left[\phi, \psi_{e}\right]+\rho_{s}^{2}\left[\psi, t_{\| e}+n_{e}\right]=0, \\
& \frac{\partial t_{\| e}}{\partial t}+\left[\phi, t_{\| e}\right]-2\left[\psi, q_{\| e}+u_{e}\right]=0, \\
& \frac{\partial q_{\| e}}{\partial t}+\left[\phi, q_{\| e}\right]-\frac{3}{2} \frac{\rho_{s}^{2}}{d_{e}^{2}}\left[\psi, t_{\| e}\right]=0, \\
& \frac{\partial N_{i}}{\partial t}+\left[\Phi, N_{i}\right]+\left[U_{i}, \Psi\right]=0, \\
& \frac{\partial \Psi_{i}}{\partial t}+\left[\Phi, \Psi_{i}\right]+\rho_{i}^{2}\left[N_{i}, \Psi\right]=0,
\end{aligned}
$$

and are closed by the relations (210)-(211), as in the five-field model. With respect to the latter, two new fields appear, $t_{\| e}$ and $q_{\| e}$, which correspond to the electron parallel and heat flux fluctuations, respectively. The ion equations (233)-(234) consist of an isothermal reduction of Eqs. (207)-(209). The electron equations (229)-(232), on the other hand, account for a more refined closure relation, that can be obtained from the model of Ref. [24], in the absence of electron FLR effects, dissipation and background inhomogeneities. In particular, unlike the five-field model of Sec. IV C 1, equilibrium magnetic curvature is neglected.

The Hamiltonian functional of the model, in terms of the field variables $\left(n_{e}, \psi_{e}, t_{\| e}, q_{\| e}, N_{i}, \Psi_{i}\right)$ is given by

$$
\begin{aligned}
& H\left(n_{e}, \psi_{e}, t_{\| e}, q_{\| e}, N_{i}, \Psi_{i}\right) \\
& =\frac{1}{2} \int d^{2} x\left(\rho_{s}^{2} n_{e}^{2}+\rho_{i}^{2} N_{i}^{2}+\frac{\rho_{s}^{2}}{2} t_{\| e}^{2}+\frac{2}{3} d_{e}^{2} q_{\| e}^{2}+d_{e}^{2} u_{e}^{2}+d_{i}^{2} U_{i}^{2}+\left|\nabla_{\perp} \psi\right|^{2}+\Phi N_{i}-\phi n_{e}\right) .
\end{aligned}
$$

As could have been expected, the total conserved energy, if compared to (214), lacks ion temperature contributions but includes electron temperature and heat flux fluctuations. We note the factor 2 in the denominator of the term containing $t_{\| e}^{2}$. This factor is absent in the corresponding term containing $T_{\| i}^{2}$ in Eq. (214). This difference, which in the equations of motion reflects in the presence of the factor 2 in Eq. (231), has no counterpart in Eq. (209) and is ascribed to the different adiabatic indices adopted for the temperature evolution in 
the two models. The six-field model adopts an adiabatic index 3 for the electrons, whereas the five-field model takes it equal to 2 for the ions. For the five-field model, this choice was, again, constrained by the Jacobi identity, in combination with the presence of magnetic curvature effects.

With regard to the Poisson bracket, this model also possesses the direct sum structure

$$
\{F, G\}=\{F, G\}_{e}+\{F, G\}_{i}
$$

with

$$
\begin{aligned}
& \{F, G\}_{e}=\int d^{2} x\left(n_{e}\left(\left[F_{n_{e}}, G_{n_{e}}\right]+\rho_{s}^{2} d_{e}^{2}\left[F_{\psi_{e}}, G_{\psi_{e}}\right]+2\left[F_{t_{\| e}}, G_{t_{\| e}}\right]+\frac{3}{2} \frac{\rho_{s}^{2}}{d_{e}^{2}}\left[F_{q_{\| e}}, G_{q_{\| e}}\right]\right)\right. \\
& +\psi_{e}\left(\left[F_{\psi_{e}}, G_{n_{e}}\right]+\left[F_{n_{e}}, G_{\psi_{e}}\right]+2\left(\left[F_{\psi_{e}}, G_{t_{\| e}}\right]+\left[F_{t_{\| e}}, G_{\psi_{e}}\right]\right)-\frac{3}{d_{e}^{2}}\left(\left[F_{q_{\| e}}, G_{t_{\| e}}\right]+\left[F_{t_{\| e}}, G_{q_{\| e}}\right]\right)\right) \\
& +t_{\| e}\left(\left[F_{t_{\| e}}, G_{n_{e}}\right]+\left[F_{n_{e}}, G_{t_{\| e}}\right]+\rho_{s}^{2} d_{e}^{2}\left[F_{\psi_{e}}, G_{\psi_{e}}\right]\right. \\
& \left.\quad-\frac{3}{2} \rho_{s}^{2}\left(\left[F_{q_{\| e}}, G_{\psi_{e}}\right]+\left[F_{\psi_{e}}, G_{q_{\| e}}\right]\right)+4\left[F_{t_{\| e}}, G_{t_{\| e}}\right]+\frac{3}{2} \frac{\rho_{s}^{2}}{d_{e}^{2}}\left[F_{q_{\| e}}, G_{q_{\| e}}\right]\right) \\
& \left.+q_{\| e}\left(\left[F_{q_{\| e}}, G_{n_{e}}\right]+\left[F_{n_{e}}, G_{q_{\| e}}\right]-2 d_{e}^{2}\left(\left[F_{t_{\| e}}, G_{\psi_{e}}\right]+\left[F_{\psi_{e}}, G_{t_{\| e}}\right]\right)+2\left(\left[F_{q_{\| e}}, G_{t_{\| e}}\right]+\left[F_{t_{\| e}}, G_{q_{\| e}}\right]\right)\right)\right)
\end{aligned}
$$

and

$$
\{F, G\}_{i}=-\int d^{2} x\left(N_{i}\left(\left[F_{N_{i}}, G_{N_{i}}\right]+\rho_{i}^{2} d_{i}^{2}\left[F_{\Psi_{i}}, G_{\Psi_{i}}\right]\right)+\Psi_{i}\left(\left[F_{\Psi_{i}}, G_{N_{i}}\right]+\left[F_{N_{i}}, G_{\Psi_{i}}\right]\right)\right)
$$

The 2D Poisson bracket possesses six families of Casimir invariants, four of which are associated with electrons and two with ions. Their expressions are given by

$$
\begin{array}{rlrl}
C_{e j} & =\int d^{2} x \mathcal{C}_{e j}\left(G_{j}\right), & j=1, \cdots, 4, \\
C_{i 1}=\int d^{2} x \mathcal{C}_{+}\left(I_{+}\right), & C_{i 2}=\int d^{2} x \mathcal{C}_{i-}\left(I_{-}\right),
\end{array}
$$


where

$$
\begin{aligned}
& G_{1}=\psi_{e}-\frac{d_{e} \rho_{s}}{\sqrt{3+\sqrt{6}}} n_{e}-d_{e} \rho_{s} \sqrt{\frac{1}{2}+\frac{1}{\sqrt{6}}} t_{\| e}-d_{e}^{2} \sqrt{\frac{2}{3}} q_{\| e}, \\
& G_{2}=\psi_{e}+\frac{d_{e} \rho_{s}}{\sqrt{3+\sqrt{6}}} n_{e}+d_{e} \rho_{s} \sqrt{\frac{1}{2}+\frac{1}{\sqrt{6}}} t_{\| e}-d_{e}^{2} \sqrt{\frac{2}{3}} q_{\| e}, \\
& G_{3}=\psi_{e}+\frac{d_{e} \rho_{s}}{\sqrt{3-\sqrt{6}}} n_{e}-d_{e} \rho_{s} \sqrt{\frac{1}{2}-\frac{1}{\sqrt{6}}} t_{\| e}+d_{e}^{2} \sqrt{\frac{2}{3}} q_{\| e}, \\
& G_{4}=\psi_{e}-\frac{d_{e} \rho_{s}}{\sqrt{3-\sqrt{6}}} n_{e}+d_{e} \rho_{s} \sqrt{\frac{1}{2}-\frac{1}{\sqrt{6}}} t_{\| e}+d_{e}^{2} \sqrt{\frac{2}{3}} q_{\| e}, \\
& I_{ \pm}=\Psi_{i} \pm d_{i} \rho_{i} N_{i},
\end{aligned}
$$

and where $\mathcal{C}_{e 1, e 2, e 3, e 4}$ and $\mathcal{C}_{ \pm}$are arbitrary functions.

As was the case of the five-field model and for the two-fluid model with isothermal electrons, adopting the normal fields $\left(G_{1}, G_{2}, G_{3}, G_{4}, I_{+}, I_{-}\right)$as dynamical field variables, the model equations (229)-(234) take the Lagrangian advection form:

$$
\begin{aligned}
\frac{\partial G_{i}}{\partial t} & =-\left[\phi_{i}, G_{i}\right], \quad i=1, \cdots, 4, \\
\frac{\partial I_{ \pm}}{\partial t} & =-\left[\Phi_{ \pm}, I_{ \pm}\right],
\end{aligned}
$$

where

$$
\begin{array}{ll}
\phi_{1}=\phi-\sqrt{3+\sqrt{6}} \frac{\rho_{s}}{d_{e}} \psi, & \phi_{2}=\phi+\sqrt{3+\sqrt{6}} \frac{\rho_{s}}{d_{e}} \psi, \\
\phi_{3}=\phi+\sqrt{3-\sqrt{6}} \frac{\rho_{s}}{d_{e}} \psi, & \phi_{4}=\phi-\sqrt{3-\sqrt{6}} \frac{\rho_{s}}{d_{e}} \psi, \\
\Phi_{ \pm}=\Phi \mp \frac{\rho_{i}}{d_{i}} \Psi, &
\end{array}
$$

The inclusion of the parallel electron heat flux dynamics still allows for a Hamiltonian formulation. The closure adopted on the electron fluid, in particular, corresponds to setting to zero the fluctuations of the energy-weighted pressure tensor (related to the fourth order moment) or, equivalently, to assuming an expansion of the underlying distribution function in terms of Hermite polynomials (see Ref. [24] and Sec. V B) truncated at the third order polynomial.

In Ref. [35], the consequences of adopting different closures for the electron fluid, on the magnetic reconnection phenomenon, were investigated numerically. On one hand, the 
comparison between the six-field model and the four-field model of Ref. [111] allows one to investigate the effect of the heat flux and temperature fluctuations against an isothermal closure. On the other hand, we remark that in the six-field model, the evolution of the heat flux is controlled by the combination of parameters $\rho_{s}^{2} / d_{e}^{2}=\left(\beta_{e} / 2\right)\left(M / m_{e}\right)$. Indeed, from Eq. (232) one sees that, in the limit $\rho_{s}^{2} / d_{e}^{2} \rightarrow 0$, the heat flux tends to be purely advected by the $\mathbf{E} \times \mathbf{B}$ velocity. In particular, if $q_{\| e}=0$ at $t=0$, then the heat flux fluctuations will not grow. The regime $\rho_{s}^{2} \ll d_{e}^{2}$ corresponds then to the adiabatic regime, were the electron heat flux is suppressed. Varying the ratio $\rho_{s}^{2} / d_{e}^{2}$ allows the investigation of the heat flux.

A selection of the results of Ref. [35] is summarized here and the reader can refer to the original article for further details.

A magnetic reconnection process was simulated numerically with both the six-field model and the isothermal four-field model of Ref. [111] on the domain $\mathcal{D}_{\mathbf{x}}=[-\pi, \pi] \times[-2 \pi, 2 \pi]$ by perturbing, with a single helicity mode, the equilibrium

$$
\begin{aligned}
& N_{i e q}=n_{e e q}=n_{0}, \quad U_{i e q}=0, \quad \psi_{e q}(x)=\sum_{n=-11}^{11} \hat{f}_{n} e^{i n x}, \\
& t_{\| e_{e q}}=0, \quad q_{\| e_{e q}}=0
\end{aligned}
$$

where $n_{0}$ is a constant, whereas $\hat{f}_{n}$ are the Fourier coefficients of the function

$$
f(x)=0.1 / \cosh ^{2}(x)
$$

In all the simulations considered here, the values $\rho_{i}=0.2, d_{e}=0.2, d_{i}=2$ were kept fixed. Fig. 3 shows how the total energy is redistributed, during the reconnection process, according to the two models. We observe that the Hamiltonian for the isothermal four-field model corresponds to the Hamiltonian (235) in the limit $t_{\| e}=q_{\| e}=0$.

The different terms contributing to the total energy are named as follows:

$$
\begin{aligned}
& E_{\text {mag }}=\frac{1}{2} \int d^{2} x|\nabla \psi|^{2}, \quad E_{k e}=\frac{d_{e}^{2}}{2} \int d^{2} x u_{e}^{2}, \quad E_{\text {ele }}=-\frac{1}{2} \int d^{2} x \phi n_{e}, \\
& E_{\text {the }}=\frac{\rho_{s}^{2}}{2} \int d^{2} x n_{e}^{2}, \quad E_{T_{\|}}=\frac{\rho_{s}^{2}}{4} \int d^{2} x t_{\| e}^{2}, \quad E_{q_{\|}}=\frac{d_{e}^{2}}{3} \int d^{2} x q_{\| e}^{2}, \\
& E_{k i}=\frac{d_{i}^{2}}{2} \int d^{2} x U_{i}^{2}, \quad E_{\text {eli }}=\frac{1}{2} \int d^{2} x \Phi N_{i}, \quad E_{t h i}=\frac{\rho_{i}^{2}}{2} \int d^{2} x N_{i}^{2} .
\end{aligned}
$$



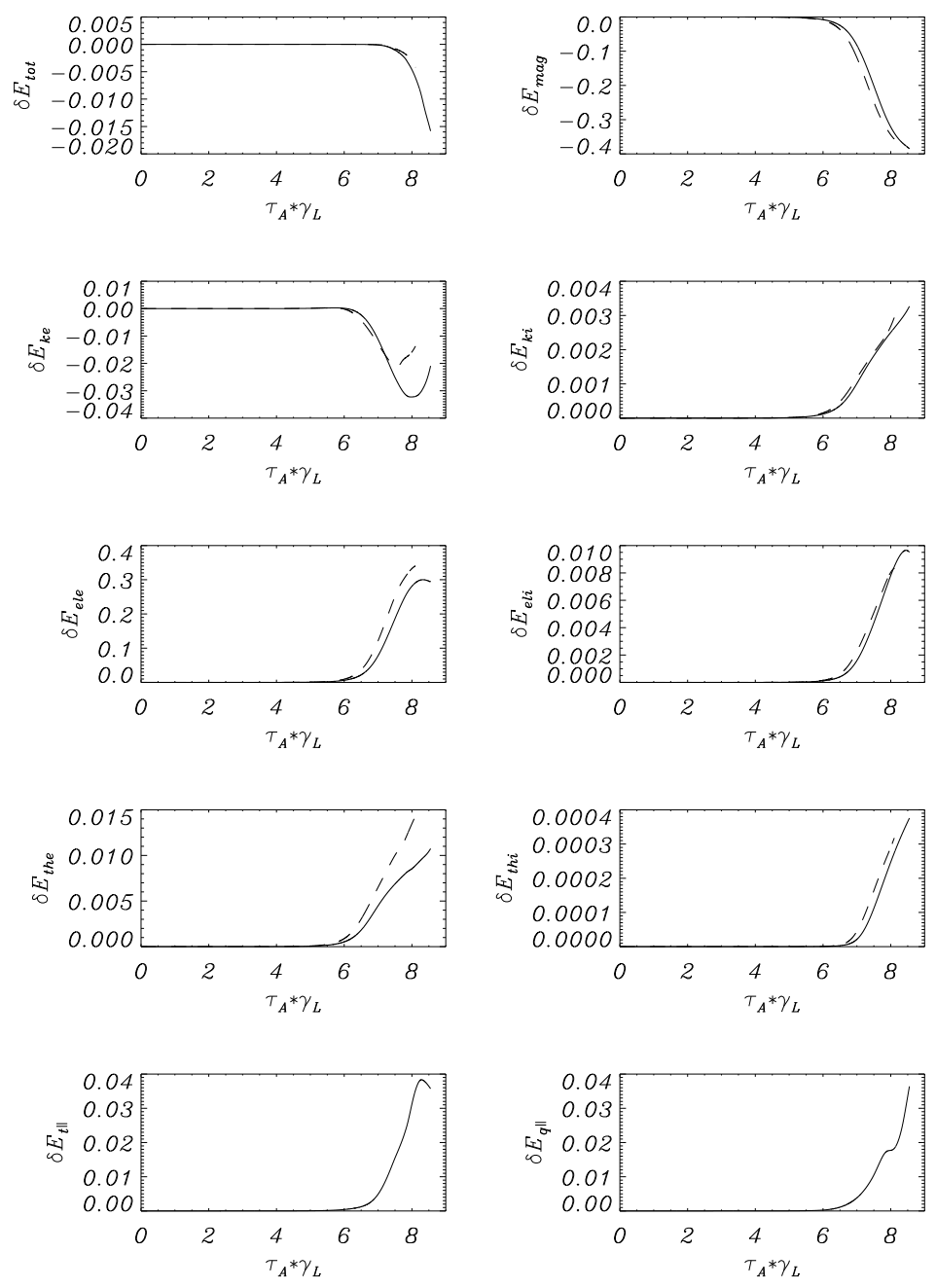

Figure 3. Relative variations of the different contributions to the total energy (Hamiltonian) for the six-field model (solid line) and for the isothermal four-field model (dashed line), for $\rho_{s}=0.1$. Time is expressed in Alfvén units normalized with respect to the linear growth rate $\gamma_{L}$ (after D. Grasso and E. Tassi, J. Plasma Phys., 81, 495810501 (2015)).

The relative variations for each form of energy, shown in Fig. 3, are calculated with respect to the initial values and normalized with respect to the total energy, so that, for instance, in the case of the magnetic energy one has $\delta E_{\text {mag }}(t)=\left(E_{\text {mag }}(t)-E_{\text {mag }}(0)\right) / H(0)$, where $H$ is the Hamiltonian functional (235).

As expected for Hamiltonian dynamics, the total energy remains essentially identical to its initial value, the only variation being due to numerical dissipation acting toward the end 
of the simulation. Magnetic energy decays as a consequence of the reconnection process, which converts it into other forms of energy. Most of the magnetic energy is transformed into electrostatic electron energy $\left(E_{\text {ele }}\right)$. The fraction of magnetic energy converted into forms of energy associated with ions $\left(E_{k i}, E_{e l i}\right.$ and $\left.E_{t h i}\right)$ is essentially the same for both closures. Therefore, modifying the closure on the electron fluid, seems to affect only the forms of energies associated with electrons. In particular, we observe that the fraction of energy feeding $E_{\text {ele }}$ and $E_{\text {the }}$ in the six-field model, is smaller than that for the four-field model. This gap in energy is clearly compensated by the new channels of free energy available in the six-field model, and corresponding to $E_{T_{\|}}$and $E_{q_{\|}}$. On the other hand, in terms of the linear growth rate, both closures yield very similar results, with slightly faster growth occurring in the six-field model [35]. The comparison with the adiabatic closure, on the other hand, can be made within the framework of the six-field model, as above said, by varying the ratio $\rho_{s}^{2} / d_{e}^{2}$. Whereas in the adiabatic regime $\rho_{s} \ll d_{e}^{2}$, heat flux fluctuations get suppressed, in the opposite, non-adiabatic regime with $\rho_{s}^{2} \gg d_{e}^{2}$, it is the temperature fluctuations which tend to damp, given that the heat can flow between different regions of plasma to equalize the temperature of the electron fluid.

Fig. 4 shows contour plots of three electron field variables in the two regimes. In the adiabatic regimes, as expected, temperature fluctuations tend to follow density fluctuations. Both tend to concentrate in four lobes with alternating signs, which turn out to be localized in correspondence of the separatrices of the magnetic island formed as a consequence of the reconnection process. In the non-adiabatic regime, density fluctuations remain essentially concentrated along the separatrices of the magnetic island, whereas temperature fluctuations, which are now much weaker, distribute inside the island. Heat flux fluctuations, which are very weak in the adiabatic regime, also tend to concentrate along the separatrices, as they become relevant in the non-adiabatic regime. 

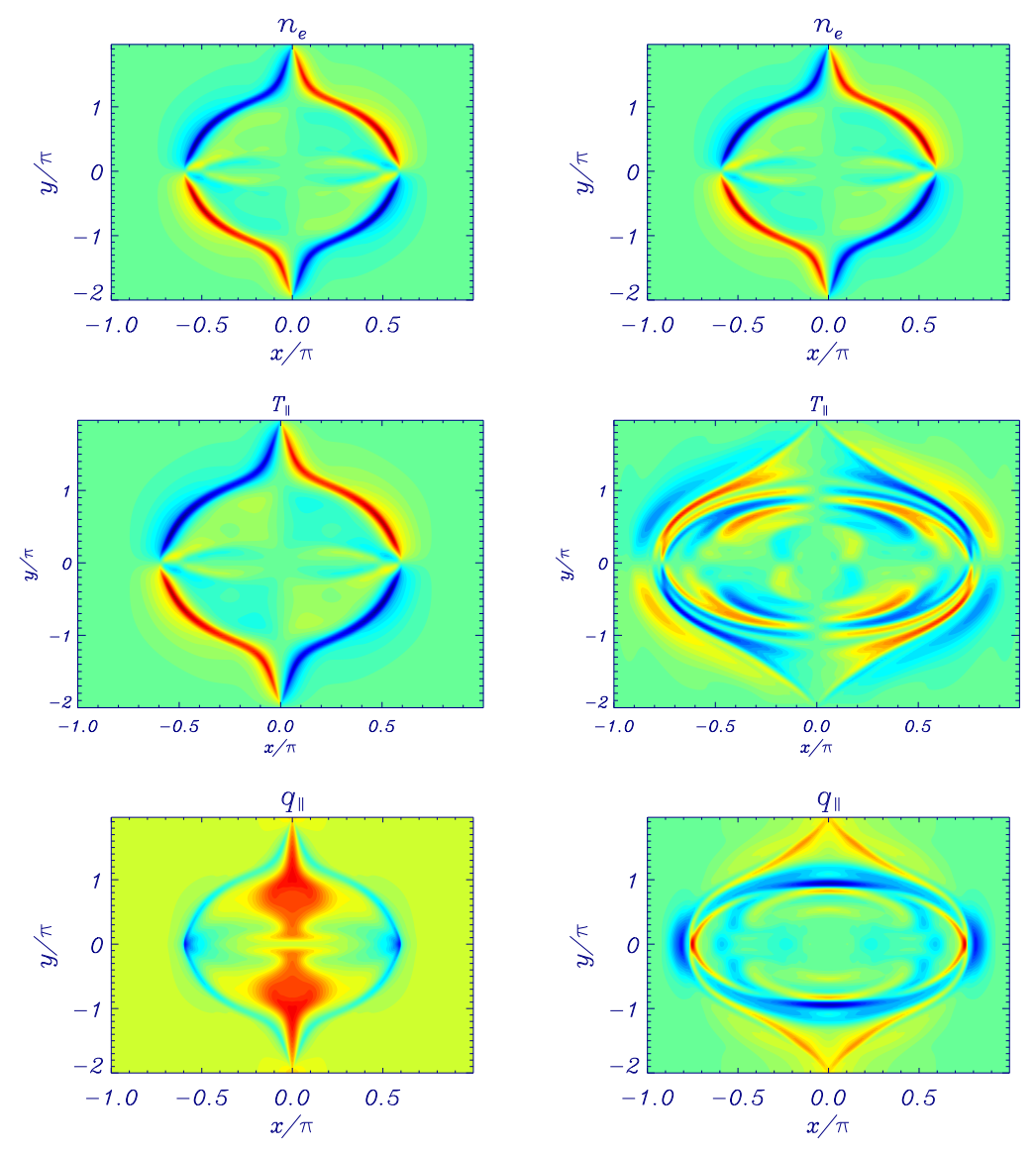

Figure 4. Contour plots of $n_{e}, t_{\| e}$ and $q_{\| e}$ in the adiabatic regime $\left(\rho_{s}=0.01\right.$, left column $)$ and in the non-adiabatic regime $\left(\rho_{s}=0.8\right.$, right column $)$ at a time into the nonlinear phase of the reconnection process (after D. Grasso and E. Tassi, J. Plasma Phys., 81, 495810501 (2015)).

\section{DERIVING HAMILTONIAN CLOSURES}

In Secs. III and IV Hamiltonian fluid models adopting different closure schemes were presented. For some of such models, in the original derivation, the Hamiltonian structure was verified a posteriori. In the cases of incompressible MHD of Sec. III B and of the gyromapped models of Sec. IV B, the models were derived from Hamiltonian parent models, by applying techniques that guarantee the existence of a Hamiltonian structure. In these cases, however, the Hamiltonian parent models were fluid models.

Adopting fluid models as parent models imposes, clearly, some limitations on the features of the models that can be derived. It would then be desirable to identify closures that provide 
fluid models with a Hamiltonian structure, starting from a parent kinetic theory, which offers a much more general approach.

Recently, a number of results were obtained in this direction. In this Section, we summarize the main achievements, which we classify according to whether the parent kinetic model includes the Vlasov equation or the drift-kinetic equation.

\section{A. Hamiltonian closures from Vlasov systems}

The Vlasov-Maxwell system is known to possess a noncanonical Hamiltonian structure $[56,114,115]$. Electrostatic reductions of the Vlasov-Maxwell system can still possess a Hamiltonian structure $[56,76,114]$. In the remaining part of Sec. V A, we consider Hamiltonian fluid models derived from Hamiltonian systems evolving a particle distribution function according to the Vlasov equation, in the absence of magnetic fields but in the presence of an external potential (in Sec. V A 1) or coupled with the evolution of the electric field (in Secs. VA 2 and VA 3).

\section{Three-moment model derived from the Vlasov equation}

We consider the following one-dimensional Vlasov equation

$$
\frac{\partial f}{\partial t}+v \frac{\partial f}{\partial x}-\frac{\partial V}{\partial x} \frac{\partial f}{\partial v}=0
$$

In Eq. (251) $f=f(x, v, t)$ is the distribution function for a population of particles subject to an external potential $V=V(x)$ (although the extension to the Vlasov-Poisson system can easily be carried out). We consider $f$ as dynamical field variable, over a domain $\mathcal{D}_{\mathbf{x}, \mathbf{v}}=$ $[0,1] \times \mathbb{R}$, with $x \in[0,1]$ and $v \in \mathbb{R}$, where $x$ and $v$ indicate the spatial and velocity coordinate, respectively. Periodic boundary conditions are assumed on the interval $0 \leq x \leq$ 1, whereas vanishing boundary conditions are imposed as $|v| \rightarrow \infty$. In this setting, Eq. (251) admits a Hamiltonian formulation with Hamiltonian

$$
H(f)=\int d x d v f\left(\frac{v^{2}}{2}+V\right)
$$


and Poisson bracket

$$
\{F, G\}=\int d x d v f\left(\frac{\partial F_{f}}{\partial x} \frac{\partial G_{f}}{\partial v}-\frac{\partial F_{f}}{\partial v} \frac{\partial G_{f}}{\partial x}\right) .
$$

As anticipated in Sec. I, the customary procedure to derive a fluid model from Eq. (251) is to first introduce the fluid moments

$$
P_{n}(x, t)=\int d v v^{n} f(x, v, t)
$$

where $n$ is a non-negative integer denoted as order of the moment.

By multiplying the left-hand side of Eq. (251) by $v^{n}$, integrating with respect to $v$ and using integration by parts, one obtains the hierarchy of fluid equations

$$
\frac{\partial P_{n}}{\partial t}+\frac{\partial P_{n+1}}{\partial x}+n \frac{\partial V}{\partial x} P_{n-1}=0
$$

for $n=0,1,2, \cdots$. Evidently, the knowledge of the evolution of the moment $P_{n}$ requires the knowledge of $P_{n+1}$, which in turn requires knowing $P_{n+2}$ and so on. Therefore, obtaining a closed fluid model evolving a finite number $N$ of moments requires imposing a closure relation

$$
P_{N}=\mathfrak{F}\left(P_{0}, P_{1}, \cdots, P_{N-1}\right)
$$

where $\mathfrak{F}$ is, in general, an operator which might depend on derivatives of the moments as well as explicitly on the spatial coordinate $x$.

Truncating the infinite hierarchy (255) by imposing a closure relation (256), however, in general does not preserve the Hamiltonian character of the parent Vlasov equation (251).

In Ref. [116] closure relations were derived in such a way that a three-moment fluid model, possessing a Hamiltonian structure by construction, could be obtained from Eq. (251).

The procedure for the derivation of such Hamiltonian closures begins by expressing the Hamiltonian structure of the parent Vlasov model in terms of the fluid moments instead of that in terms of the distribution function.

With regard to the Hamiltonian (252), this is easily accomplished by substitution and yields

$$
H\left(P_{0}, P_{1}, P_{2}\right)=\int d x\left(\frac{P_{2}}{2}+V P_{0}\right) .
$$


As far as the Poisson bracket (253) is concerned, it is necessary to be able to express the functional derivatives with respect to $f$, in terms of functional derivatives with respect to $P_{0}, P_{1}, P_{2}, \cdots$. This is accomplished by making use of the functional chain rule described in Sec. II applied to the change of variables $f \rightarrow\left(P_{0}, P_{1}, P_{2}, \cdots\right)$. Noticing that, from the relation (254) one obtains the relation $\delta P_{n}=\int d v v^{n} \delta f$ between the variations, the functional chain rule yields

$$
\frac{\delta F}{\delta f}=\sum_{i=0}^{+\infty} \frac{\delta \bar{F}}{\delta P_{i}}
$$

Using this relation in (253) one obtains that the Vlasov Poisson bracket in terms of the fluid moments reads

$$
\{F, G\}=\sum_{m, n=0}^{+\infty} \int d x P_{m+n-1}\left(n G_{n} \partial_{x} F_{m}-m F_{m} \partial_{x} G_{n}\right)
$$

where the subscripts on the functionals indicates the order of the moments, so that, for instance, $F_{m}=\delta F / \delta P_{m}$. The bracket (259) was derived in Ref. [117] and associated with the Vlasov-Poisson bracket in Ref. [118].

In order to derive a Hamiltonian three-moment model, a natural choice of the field variables is clearly the set $\left(P_{0}, P_{1}, P_{2}\right)$. If one evaluates the Poisson bracket $(259)$ restricting to functionals $F$ and $G$ depending on $P_{0}, P_{1}$ and $P_{2}$ only, one obtains

$$
\{F, G\}=\{F, G\}_{J}+\{F, G\}^{*}
$$

where

$$
\begin{aligned}
& \{F, G\}_{J}=\int d x\left(P_{0}\left(G_{1} \partial_{x} F_{0}-F_{1} \partial_{x} G_{0}\right)+P_{1}\left(G_{1} \partial_{x} F_{1}-F_{1} \partial_{x} G_{1}\right)\right. \\
& \left.+P_{2}\left(G_{1} \partial_{x} F_{2}-F_{1} \partial_{x} G_{2}\right)\right) \\
& \{F, G\}^{*}=2 \int d x\left(P_{1}\left(G_{2} \partial_{x} F_{0}-F_{2} \partial_{x} G_{0}\right)+P_{2}\left(G_{2} \partial_{x} F_{1}-F_{2} \partial_{x} G_{1}\right)\right. \\
& \left.+P_{3}\left(G_{2} \partial_{x} F_{2}-F_{2} \partial_{x} G_{2}\right)\right) .
\end{aligned}
$$

From Eq. (261) it emerges then that, although $F$ and $G$ depend only on $P_{0}, P_{1}$ and $P_{2}$, the bracket $\{F, G\}$ does not, because it depends explicitly on $P_{3}$. This differs, in particular, from the case where one restricts to the set of functionals of $P_{0}$ and $P_{1}$, which, on the contrary, 
forms a sub-algebra $[119,120]$. As will become evident later, the case of the sub-algebra of functionals of $P_{0}$ and $P_{1}$ includes the case of barotropic fluids. In order for the expression (260) to be a functional of $P_{0}, P_{1}$ and $P_{2}$ we impose a closure relation

$$
P_{3}=\mathcal{F}\left(x, P_{0}, P_{1}, P_{2}\right),
$$

expressing the fact that $P_{3}$ must depend on its lower order moments, and possibly also on the coordinate $x$ through a function $\mathcal{F}$ (we assume no dependence on the derivatives of $P_{0}, P_{1}$ and $P_{2}$, on the other hand). However, the expression that one obtains after inserting (262) into (260) in general is not a Poisson bracket. Indeed, whereas bilinearity, antisymmetry and the Leibniz identity are satisfied for any $\mathcal{F}$, the Jacobi identity is not. Imposing the Jacobi identity (note that the $\{,\}_{J}$ satisfies the Jacobi identity independently) implies the following constraints on $\mathcal{F}$ :

$$
\begin{aligned}
P_{0} \frac{\partial \mathcal{F}}{\partial P_{1}}+2 P_{1} \frac{\partial \mathcal{F}}{\partial P_{2}}-3 P_{2} & =0 \\
P_{0} \frac{\partial \mathcal{F}}{\partial P_{0}}+2 P_{1} \frac{\partial \mathcal{F}}{\partial P_{1}}-4 \mathcal{F}+3 P_{2} \frac{\partial \mathcal{F}}{\partial P_{2}} & =0 \\
\frac{\partial \mathcal{F}}{\partial x} & =0 .
\end{aligned}
$$

Equation (265) implies that $\mathcal{F}$ cannot depend explicitly on $x$. The remaining two equations are solved by the method of characteristics in Ref. [116] and yield the following Hamiltonian closure

$$
P_{3}=3 \frac{P_{1} P_{2}}{P_{0}}-2 \frac{P_{1}^{3}}{P_{0}^{2}}+\frac{P_{0}^{4}}{2} \mathcal{Q}\left(\frac{P_{2}}{P_{0}^{3}}-\frac{P_{1}^{2}}{P_{0}^{4}}\right),
$$

where $\mathcal{Q}$ is an arbitrary function. The Poisson bracket (260), with $P_{3}$ given by Eq. (266), and the Hamiltonian (257) generate a Hamiltonian three-moment model, possessing a free function $\mathcal{Q}$.

This Hamiltonian structure can be expressed in an alternative, physically relevant form 
as

$$
\begin{aligned}
& H(\rho, M, U)=\int d x\left(\frac{M^{2}}{2 \rho}+\rho U+\rho V\right) \\
& \{F, G\}=-\int d x\left(\rho\left(F_{M} \partial_{x} G_{\rho}-G_{M} \partial_{x} F_{\rho}\right)+M\left(F_{M} \partial_{x} G_{M}-G_{M} \partial_{x} F_{M}\right)\right. \\
& \left.+U\left(F_{M} \partial_{x} G_{U}-G_{M} \partial_{x} F_{U}+F_{U} \partial_{x} G_{M}-G_{U} \partial_{x} F_{M}\right)-\rho^{2} \mathcal{Q}\left(2 U / \rho^{2}\right)\left(F_{U} \partial_{x} G_{U}-G_{U} \partial_{x} F_{U}\right)\right)
\end{aligned}
$$

where the new field variables $\rho, M$ and $U$ are related to the previous moment variables by

$$
\rho=P_{0}, \quad M=P_{1}, \quad U=\frac{1}{2 P_{0}}\left(P_{2}-\frac{P_{1}^{2}}{P_{0}}\right) .
$$

The variables $\rho, M$ and $U$ represent the density, the momentum density and the internal energy of the fluid, respectively. The Hamiltonian (267) and the Poisson bracket (269) can be compared with the corresponding expressions (40) and (41), for a barotropic fluid. Indeed, one sees that the Hamiltonian structure for a 1D barotropic fluid can be obtained from Eqs. (267)-(269) in the absence of potential $(V=0)$, restricting to functionals of $\rho$ and $M$ and imposing that the internal energy be a function of the density. In the three-moment model, on the other hand, the internal energy is an independent dynamical variable.

The equations of motion for the three-moment model can be written as

$$
\begin{aligned}
& \frac{\partial \rho}{\partial t}+\frac{\partial}{\partial x}(\rho \mathrm{v})=0 \\
& \frac{\partial \mathrm{v}}{\partial t}+\mathrm{v} \frac{\partial \mathrm{v}}{\partial x}+\frac{\partial V}{\partial x}+\frac{1}{\rho} \frac{\partial p}{\partial x}=0 \\
& \frac{\partial U}{\partial t}+\mathrm{v} \frac{\partial U}{\partial x}+2 U \frac{\partial \mathrm{v}}{\partial x}+\frac{1}{\rho} \frac{\partial}{\partial x}\left(\rho^{4} \mathcal{Q}\right)=0
\end{aligned}
$$

where we replaced the momentum variable $M$ in favor of the velocity field $\mathrm{v}=M / \rho$ and where we defined the pressure $p=2 \rho U$, which is consistent with the relation $\rho U=p /(\gamma-1)$, valid for a one-dimensional gas with adiabatic index $\gamma=3$.

Eq. (273) can also be expressed as the following evolution equation for the pressure:

$$
\frac{\partial p}{\partial t}+\mathrm{v} \frac{\partial p}{\partial x}+3 p \frac{\partial \mathrm{v}}{\partial x}+2 \frac{\partial}{\partial x}\left(\rho^{4} \mathcal{Q}\right)=0
$$

The family of models (271)-(273) represent then extensions to a higher order closure with respect to a barotropic fluid, and still possessing a Hamiltonian structure. Note that, in 
order for the model to be Hamiltonian, $\mathcal{Q}$ must be $\mathcal{Q}\left(2 U / \rho^{2}\right)$. From Eq. (274) it follows that $q=\rho^{4} \mathcal{Q}$ corresponds to the heat flux.

The particular case $\mathcal{Q}=0$ corresponds to a situation where the underlying Vlasov distribution function has zero skewness, as in the case of a Maxwellian distribution function

$$
f(x, v)=\frac{\rho}{\sqrt{4 \pi U}} \exp \left(-\frac{(v-M / \rho)^{2}}{4 U}\right) .
$$

In this limit, the system is constrained by the famly of Casimir invariants

$$
C=\int d x \rho \kappa(S)
$$

where $S=2 U / \rho^{2}$ and $\kappa$ is an arbitrary function.

In the limit $\mathcal{Q}=0$, the Poisson bracket (269), expressed in terms of the variables $\rho, M$ and $S$ is invariant under the transformation $S \rightarrow s$, where $s=f(S)$, for an arbitrary but invertible function $f$. The model equations admit then also the formulation

$$
\begin{aligned}
& \frac{\partial \rho}{\partial t}+\frac{\partial}{\partial x}(\rho \mathrm{v})=0 \\
& \frac{\partial \mathrm{v}}{\partial t}+\mathrm{v} \frac{\partial \mathrm{v}}{\partial x}+\frac{\partial V}{\partial x}+\frac{1}{\rho} \frac{\partial}{\partial x}\left(\rho^{3} g(s)\right)=0 \\
& \frac{\partial s}{\partial t}+\mathrm{v} \frac{\partial s}{\partial x}=0
\end{aligned}
$$

where $g=f^{-1}$. By identifying $s$ with the entropy per unit mass, one sees that the case $\mathcal{Q}=0$ corresponds to an adiabatic fluid governed by a polytropic equation of state $p=g(s) \rho^{3}$ (one could also compare Eqs. (277)-(279) with Eqs. (92), (93), (95) in the absence of magnetic field).

If $\mathcal{Q} \neq 0$, on the other hand, the underlying distribution function is allowed a finite skewness. Adiabatic processes in this case are restricted to cases where $\mathcal{Q}$ has a zero. If $\mathcal{Q}$ does not vanish on its domain, then the system possesses three Casimir invariants, corresponding to

$$
C_{1}=\int d x \rho, \quad C_{2}=\int d x\left(\frac{M}{\rho}-\frac{\rho \kappa_{0}^{2}(S)}{4}\right), \quad C_{3}=\int d x \rho \kappa_{0}(S)
$$

where $\kappa_{0}$ is such that $\kappa_{0}^{\prime}=1 / \sqrt{|\mathcal{Q}|}$. 


\section{A four-moment model derived from the Vlasov-Ampère system}

We consider here the dynamics of a population of charged particles, in the presence of a neutralizing background composed of static particles with opposite charge and subject to the action of the resulting electric field. Without loss of generality, in the plasma that we are considering, the population of dynamical particles is composed of electrons, with a background population of static ions. This system can be described, in normalized units, by the following Vlasov-Ampère model :

$$
\begin{aligned}
& \frac{\partial f}{\partial t}+v \frac{\partial f}{\partial x}-\tilde{E} \frac{\partial f}{\partial v}=0, \\
& \frac{\partial E}{\partial t}+\tilde{j}=0,
\end{aligned}
$$

where $f=f(x, v, t)$ is the electron distribution function, $E=E(x, t)$ is the electric field, $j=-\int d v v f$ is the current density. Here the tilde indicates the fluctuating part with respect to the spatial average value, so that $\tilde{E}=E-\int d x E$ and $\tilde{j}=j-\int d x j$.

The system (281)-(282) admits a Hamiltonian formulation in terms of the dynamical variables $(f, E)$. We assume that the coordinate domain of the distribution function $f$ is $\mathcal{D}_{\mathbf{x}, \mathbf{v}}=[0,1] \times \mathbb{R}$, whereas that for the electric field $E$ is $\mathcal{D}_{\mathbf{x}}=[0,1]$. As in the previous cases, periodicity is imposed at $x=0$ and $x=1$, whereas vanishing boundary conditions are assumed for $|v| \rightarrow \infty$. Note that this case refers to the situation, mentioned in Sec. II, where the dynamical field variables depend on different domains of coordinates.

The Hamiltonian for the Vlasov-Ampère system is given by

$$
H(f, E)=\int d x d v f \frac{v^{2}}{2}+\int d x \frac{E^{2}}{2},
$$

where the first term on the right-hand side corresponds to the kinetic energy, and the second term to the electrostatic energy.

The expression for the Poisson bracket is given by

$$
\begin{aligned}
& \{F, G\}=\int d x d v f\left(\frac{\partial F_{f}}{\partial x} \frac{\partial G_{f}}{\partial v}-\frac{\partial F_{f}}{\partial v} \frac{\partial G_{f}}{\partial x}\right. \\
& \left.+\left(\widetilde{F_{E}} \frac{\partial G_{f}}{\partial v}-\widetilde{G_{E}} \frac{\partial F_{f}}{\partial v}\right)\right) .
\end{aligned}
$$


Note that the contribution (284) corresponds to the Poisson bracket (253) of the case with external potential, whereas the terms (285) account for the evolution of the electric field.

In Ref. [121], the Hamiltonian closures for a four-moment fluid model, derived from Eqs. (281)-(282) and evolving the field variables $\left(P_{0}, P_{1}, P_{2}, P_{3}, E\right)$, were found.

The approach is similar to that of Ref. [116], although a higher order closure is considered here. The Hamiltonian (283) can easily be expressed in terms of the involved field variables $\left(P_{0}, P_{1}, P_{2}, P_{3}, E\right)$ as

$$
H\left(P_{0}, P_{1}, P_{2}, P_{3}, E\right)=\frac{1}{2} \int d x\left(P_{2}+E^{2}\right) .
$$

In terms of the fluid moments and electric field, on the other hand, the Poisson bracket (284)-(285) becomes

$$
\{F, G\}=\sum_{m, n=0}^{+\infty} \int d x\left(P_{m+n-1}\left(n G_{n} \partial_{x} F_{m}-m F_{m} \partial_{x} G_{n}\right)+P_{m-1}\left(G_{m} \widetilde{F_{E}}-F_{m} \widetilde{G_{E}}\right)\right) .
$$

The bracket (287) is then evaluated on functionals $F$ and $G$ depending on $P_{0}, P_{1}, P_{2}, P_{3}$ and $E$. The result is a functional that depends also on $P_{4}$ and $P_{5}$. A closure relation is thus required, and in this case, unlike for the three-moment model of Sec. V A 1, it involves two higher order moments rather than one. Imposing $P_{4}=P_{4}\left(P_{0}, P_{1}, P_{2}, P_{3}, E\right)$ and $P_{5}=P_{5}\left(P_{0}, P_{1}, P_{2}, P_{3}, E\right)$ in Eq. (287) (we exclude dependence on the derivatives of the moments and on the spatial coordinate $x$ ) one looks for the constraints that $P_{4}$ and $P_{5}$ need to satisfy in order for the Jacobi identity to be verified. The conditions on the fluid moments $P_{4}$ and $P_{5}$ can be more conveniently expressed in terms of the reduced fluid moments defined as $S_{n}(x, t)=\left(1 / \rho^{n+1}(x, t)\right) \int d v(v-\mathrm{v}(x, t))^{n} f(x, v, t)$, for $n \geq 2$. In Ref. [121], it is shown that a necessary condition for the Jacobi identity to be satisfied is that $S_{4}$ and $S_{5}$ not depend on $\rho, \mathrm{v}$ and $E$. Also, the constraints on $S_{4}$ fully determine the closure on $S_{5}$. The constraints reduce to the following two partial differential equations:

$$
\begin{aligned}
& 4 S_{3} \frac{\partial^{2} S_{4}}{\partial S_{2}^{2}}-\frac{\partial^{2} S_{4}}{\partial S_{2} \partial S_{3}}\left(9 S_{2}^{2}-5 S_{4}\right)-\frac{\partial S_{4}}{\partial S_{2}} \frac{\partial S_{4}}{\partial S_{3}}=12 S_{3} \\
& 4 S_{3} \frac{\partial^{2} S_{4}}{\partial S_{3} \partial S_{2}}-\frac{\partial^{2} S_{4}}{\partial S_{3}^{2}}\left(9 S_{2}^{2}-5 S_{4}\right)+12 S_{2}=\left(\frac{\partial S_{4}}{\partial S_{3}}\right)^{2}+2 \frac{\partial S_{4}}{\partial S_{2}},
\end{aligned}
$$

to be solved for $S_{4}=S_{4}\left(S_{3}, S_{2}\right)$. 
Solutions are sought in such a way that the relation between $S_{4}, S_{2}$ and $S_{3}$ does not involve further dimensional parameters. With this assumption, given that the relation $S_{4}=$ $S_{4}\left(S_{2}, S_{3}\right)$ involves three quantities and a single physical dimensions $L^{2} T^{-1}$ (with $L$ and $T$ indicating the units of length and time, respectively), the Buckingham $\pi$ theorem [122] implies that the relation $S_{4}=S_{4}\left(S_{2}, S_{3}\right)$ can be reduced to a relation $\zeta=R(\xi)$ between two dimensionless quantities $\zeta$ and $\xi$. Setting $\zeta=S_{4} / S_{2}^{2}$ and $\xi=S_{3} / S_{2}^{3 / 2}$, two solutions for the closure relation were found. We focus on one of these two solutions, which leads to a physically relevant model, and which corresponds to

$$
S_{4}=S_{2}^{2}+\frac{S_{3}^{2}}{S_{2}}, \quad S_{5}=2 S_{2} S_{3}+\frac{S_{3}^{2}}{S_{2}^{2}}
$$

The resulting model equations can be written in the following form:

$$
\begin{aligned}
& \frac{\partial \rho}{\partial t}+\frac{\partial}{\partial x}(\rho \mathrm{v})=0 \\
& \frac{\partial \mathrm{v}}{\partial t}+\mathrm{v} \frac{\partial \mathrm{v}}{\partial x}+\frac{1}{\rho} \frac{\partial p}{\partial x}+\tilde{E}=0 \\
& \frac{\partial p}{\partial t}+\mathrm{v} \frac{\partial p}{\partial x}+3 p \frac{\partial \mathrm{v}}{\partial x}+2 \frac{\partial q}{\partial x}=0 \\
& \frac{\partial q}{\partial t}+\mathrm{v} \frac{\partial q}{\partial x}+4 q \frac{\partial \mathrm{v}}{\partial x}+2 \frac{\partial}{\partial x}\left(\frac{q^{2}}{p}\right)-\frac{1}{4 \rho^{3}} \frac{\partial}{\partial x}\left(\rho^{2} p^{2}\right)=0 \\
& \frac{\partial E}{\partial t}-\widetilde{\rho \mathrm{v}}=0
\end{aligned}
$$

where

$$
p=\rho^{3} S_{2}=P_{2}-\frac{P_{1}^{2}}{P_{0}}, \quad q=\frac{\rho^{4}}{2} S_{3}=\frac{P_{3}}{2}-\frac{3}{2} \frac{P_{1} P_{2}}{P_{0}}+\frac{P_{1}^{3}}{P_{0}^{2}},
$$

correspond to the pressure and to the heat flux, respectively.

The closure on $P_{4}$ yielding Eqs. (291)-(294) corresponds to a bi-delta reduction [123-126], i.e. to a distribution function of the form $f(x, v, t)=\rho_{1}(x, t) \delta\left(v-\mathrm{v}_{1}(x, t)\right)+\rho_{2}(x, t) \delta(v-$ $\left.\mathrm{v}_{2}(x, t)\right)$, consisting of two beams centered at the velocities $\mathrm{v}_{1}$ and $\mathrm{v}_{2}$ and distributed in space according to the densities $\rho_{1}$ and $\rho_{2}$, respectively.

Global Casimir invariants for this system correspond to

$$
\begin{array}{r}
C_{1}=\int d x \rho, \quad C_{2}=\int d x E, \quad C_{3}=\int d x \overline{\mathrm{v}}, \\
C_{4}=\int d x Q_{2}, \quad C_{5}=\int d x Q_{3},
\end{array}
$$


where

$$
\overline{\mathrm{v}}=\mathrm{v}+\frac{q}{p} \quad Q_{2}=2 \sqrt{\frac{p}{\rho}+\frac{q^{2}}{p^{2}}}, \quad Q_{3}=q \rho \sqrt{\frac{\rho}{p^{3}+\rho q^{2}}}
$$

The set of variables $\left(\rho, E, \overline{\mathrm{v}}, Q_{2}, Q_{3}\right)$ identifies a set of normal fields, in terms of which the Poisson bracket for the model (291)-(295) takes the remarkably simple form

$$
\{F, G\}=\int d x\left(G_{\overline{\mathrm{v}}} \frac{\partial F_{\rho}}{\partial x}-F_{\overline{\mathrm{v}}} \frac{\partial G_{\rho}}{\partial x}+\left(G_{\overline{\mathrm{v}}} \widetilde{F_{E}}-F_{\overline{\mathrm{v}}} \widetilde{G_{E}}\right)-2 G_{Q_{3}} \frac{\partial F_{Q_{2}}}{\partial x}+2 F_{Q_{3}} \frac{\partial G_{Q_{2}}}{\partial x}\right)
$$

As was the case for the three-moment model, also in this case one Casimir invariant, namely $C_{3}$ corresponds to the integral of a generalized velocity $\overline{\mathrm{v}}$ (analogous to $C_{2}$ in Eq. (280))

In addition to the global invariants $C_{1}, \cdots, C_{5}$, the system possesses also a local Casimir invariant

$$
C_{6}(x)=\frac{\partial E(x)}{\partial x}+\rho(x)
$$

which expresses Gauss's law governing the charge density.

Interestingly, Eqs. (288)-(289) possess, still in the framework of the above dimensional analysis, a second solution providing a second Hamiltonian closure. This second solution, however, does not allow for symmetric distribution functions, and consequently, its physical relevance for plasma physics remains questionable. Also, this second solution yields a Poisson bracket without a complete set normal fields [121].

3. Higher-order Hamiltonian closures for the models derived from the Vlasov-Ampère system

In Sec. V A 2 we summarized the results of Ref. [121], where it was found that, in the framework of the above discussed dimensional analysis, and excluding in the closure relation any dependence on the spatial coordinates as well as on derivatives of the moments, only two Hamiltonian closures exist for four-moment models derived from the Vlasov-Ampère system. In Ref. [127] it was shown, on the other hand, that there exist a family of closures of the Vlasov-Ampère system, those associated with the so called water-bag distribution functions, which lead to Hamiltonian fluid models for an arbitrary number of moments. 
Given a non-negative integer $N$, the $N$-water-bag distribution function [128-130] is defined as

$$
f_{N}(x, v, t)=\sum_{i=1}^{N+1} a_{i} \Theta\left[v-\mathrm{v}_{i}(x, t)\right],
$$

where $\Theta$ is the Heaviside distribution, $a_{1}, \cdots, a_{N+1}$ are constants and $\mathrm{v}_{1}, \cdots, \mathrm{v}_{N+1}$ are contour velocity fields identifying the boundaries between the "bags". In order for the distribution to have a compact support, the constraint $\sum_{i=1}^{N+1} a_{i}=0$ is imposed, Moreover, it is assumed that $\mathrm{v}_{1}(x, t)<\mathrm{v}_{2}(x, t)<\cdots<\mathrm{v}_{N+1}(x, t)$ for all $(x, t) \in\left[0 ; 1\left[\times \mathbb{R}_{+}\right.\right.$.

Water-bag distribution functions can be considered as piecewise constant approximations of smooth distribution functions and were used, for instance, in the context of gyrokinetic theory [131-134]. An important feature of the water-bag distribution functions is that they provide a weak solution of the Vlasov-Ampère system (281)-(282) if and only if the velocity fields $\mathrm{v}_{1}, \cdots, \mathrm{v}_{N+1}$ satisfy

$$
\frac{\partial \mathrm{v}_{i}}{\partial t}=-\mathrm{v}_{i} \frac{\partial \mathrm{v}_{i}}{\partial x}-\widetilde{E}
$$

for all $1 \leq i \leq N+1$ and

$$
\frac{\partial E}{\partial t}=-\frac{1}{2} \sum_{i=1}^{N+1} a_{i} \widetilde{\mathrm{v}}_{i}^{2}
$$

By solving the fluid-like system (303)-(304), one then finds a solution of the Vlasov-Ampère system of the form (302).

The system (303)-(304) possesses a Hamiltonian structure [135-137] with Hamiltonian

$$
H\left(\mathrm{v}_{1}, \cdots, \mathrm{v}_{N+1}, E\right)=\frac{1}{2} \int d x\left(-\frac{1}{3} \sum_{i=1}^{N+1} a_{i} \mathrm{v}_{i}^{3}+E^{2}\right),
$$

and Poisson bracket

$$
\{F, G\}=\sum_{i=1}^{N+1} \int d x\left(\frac{1}{a_{i}} F_{\mathrm{v}_{i}} \frac{\partial}{\partial x} G_{\mathrm{v}_{i}}+G_{\mathrm{v}_{i}} \widetilde{F_{E}}-F_{\mathrm{v}_{i}} \widetilde{G_{E}}\right) .
$$

As above anticipated, water-bag distribution functions (302) lead to Hamiltonian fluid models for an arbitrary number of moments.

This is easily seen in the case of a single water-bag distribution function $f_{1}(x, v, t)=$ $\Theta\left[v-\mathrm{v}_{1}(x, t)\right]-\Theta\left[v-\mathrm{v}_{2}(x, t)\right]$, which corresponds to a two-moment model, with

$$
P_{0}=\mathrm{v}_{2}-\mathrm{v}_{1}, \quad P_{1}=\frac{\mathrm{v}_{2}^{2}-\mathrm{v}_{1}^{2}}{2} .
$$


The contour velocity fields are related to the density $\rho$ and to the velocity $\mathrm{v}$ of the fluid by

$$
\mathrm{v}_{1}=\mathrm{v}-\frac{\rho}{2}, \quad \mathrm{v}_{2}=\mathrm{v}+\frac{\rho}{2}
$$

The Hamiltonian and the Poisson bracket for the model are given by

$$
H(\rho, \mathrm{v}, E)=\int d x\left(\rho \frac{\mathrm{v}^{2}}{2}+\rho U(\rho)+\frac{E^{2}}{2}\right)
$$

and

$$
\{F, G\}=-\int d x\left(F_{\rho} \frac{\partial G_{\mathrm{v}}}{\partial x}-G_{\rho} \frac{\partial F_{\mathrm{v}}}{\partial x}+F_{\mathrm{v}} \widetilde{G_{E}}-G_{\mathrm{v}} \widetilde{F_{E}}\right),
$$

respectively. In the Hamiltonian (307), the expression for the internal energy corresponds to $U(\rho)=\rho^{2} / 24$. The corresponding equations of motion read

$$
\begin{aligned}
& \frac{\partial \rho}{\partial t}+\frac{\partial}{\partial x}(\rho \mathrm{v})=0 \\
& \frac{\partial \mathrm{v}}{\partial t}+\mathrm{v} \frac{\partial \mathrm{v}}{\partial x}+\frac{1}{\rho} \frac{\partial p}{\partial x}+\tilde{E}=0 \\
& \frac{\partial E}{\partial t}-\widetilde{\rho \mathrm{v}}=0
\end{aligned}
$$

where the expression for the pressure is given by $p=\rho^{3} / 12$. Equivalently, this closure can be written as

$$
P_{2}=\frac{P_{1}^{2}}{P_{0}}+\frac{P_{0}^{3}}{12}
$$

in terms of the fluid moments, or as $S_{2}=1 / 12$ in terms of reduced moments.

It follows then that the single water-bag case leads to the equations for a barotropic fluid, governed by an adiabatic equation of state for an ideal gas with one degree of freedom, and interacting with an electric field.

Associated Casimir invariants are given by:

$$
C_{1}=\int d x \rho, \quad C_{2}=\int d x E, \quad C_{3}=\int d x \mathrm{v}, \quad C_{4}(x)=\frac{\partial E}{\partial x}+\rho .
$$

The invariant $C_{1}, C_{2}$ and $C_{4}$ are in common with the Casimir invariants of the four-moment model (291)-(295). The conservation of the mean value of the fluid velocity, associated with $C_{2}$, on the other hand, is peculiar to the adiabatic closure of this two-moment model. 
In the general case of an $N$-water-bag distribution function, the fluid density and velocity are given by

$$
\rho=-\sum_{i=1}^{N+1} a_{i} \mathrm{v}_{i}, \quad \mathrm{v}=\frac{\sum_{i=1}^{N+1} a_{i} \mathrm{v}_{i}^{2}}{2 \sum_{k=1}^{N+1} a_{k} \mathrm{v}_{k}} .
$$

It is then convenient to introduce the quantities

$$
n_{l}=-\frac{\left(\mathrm{v}_{l+1}-\mathrm{v}_{l}\right) \sum_{i=1}^{l} a_{i}}{\sum_{k=1}^{N+1} a_{k} \mathrm{v}_{k}}, \quad 1 \leq l \leq N-1,
$$

such that $\rho n_{i}$ is the density of particles contained in the $i$-th bag for $1 \leq i \leq N-1$, and

$$
\nu_{i}=\sum_{k=1}^{i} n_{k}, \quad 1 \leq i \leq N-1 .
$$

From Eq. (315), it follows that the quantity $\int d x \rho \nu_{i}$ corresponds to the cumulative number of particles contained in the first $i$ bags.

In terms of the field variables $\left(\rho, \mathrm{v}, \nu_{1}, \cdots, \nu_{N-1}, E\right)$, the Hamiltonian structure of the $N+1$-moment model associated with an $N$-water-bag distribution function consists of

$$
\begin{aligned}
& H\left(\rho, \mathrm{v}, \nu_{1}, \cdots, \nu_{N-1}, E\right)=\frac{1}{2} \int d x\left(\rho \mathrm{v}^{2}+\rho^{3} S_{2}\left(\nu_{1}, \cdots, \nu_{N-1}\right)+E^{2}\right), \\
& \{F, G\}=-\int d x\left(F_{\rho} \frac{\partial G_{\mathrm{v}}}{\partial x}-G_{\rho} \frac{\partial F_{\mathrm{v}}}{\partial x}+F_{\mathrm{v}} \widetilde{G_{E}}-G_{\mathrm{v}} \widetilde{F_{E}}\right. \\
& \left.+\frac{1}{\rho}\left(F_{\nu_{i}} G_{\mathrm{v}}-G_{\nu_{i}} F_{\mathrm{v}}\right) \frac{\partial \nu_{i}}{\partial x}-\lambda_{i} \frac{F_{\nu_{i}}}{\rho} \frac{\partial}{\partial x}\left(\frac{G_{\nu_{i}}}{\rho}\right)\right)
\end{aligned}
$$

In the Hamiltonian (317), the expression for $S_{2}$ in terms of the field variables $\nu_{1}, \cdots, \nu_{N-1}$ can be obtained from the general expression for the reduced moments, corresponding to

$$
S_{i}(x, t)=-\frac{1}{(i+1) \rho^{i+1}} \sum_{k=1}^{N+1} a_{k}\left[\mathrm{v}_{k}(x, t)-\mathrm{v}(x, t)\right]^{i+1},
$$

for $i \geq 2$.

In the Poisson bracket (318), the sum of repeated indices from 1 to $N-1$ is understood and we introduced the constants

$$
\lambda_{i}=\frac{\sum_{k=1}^{i} a_{k} \sum_{l=1}^{i+1} a_{l}}{a_{i+1}}
$$


The resulting $N+1$ moment model equations are given by

$$
\begin{aligned}
& \frac{\partial \rho}{\partial t}+\frac{\partial}{\partial x}(\rho \mathrm{v})=0 \\
& \frac{\partial \mathrm{v}}{\partial t}+\mathrm{v} \frac{\partial \mathrm{v}}{\partial x}+\frac{1}{\rho} \frac{\partial p}{\partial x}+\tilde{E}=0 \\
& \frac{\partial \nu_{i}}{\partial t}+\mathrm{v} \frac{\partial \nu_{i}}{\partial x}-\frac{\lambda_{i}}{\rho} \frac{\partial}{\partial x}\left(\frac{\rho^{2}}{2} \frac{\partial S_{2}}{\partial \nu_{i}}\right)=0, \quad 1 \leq i \leq N-1 \\
& \frac{\partial E}{\partial t}-\widetilde{\rho \mathrm{v}}=0
\end{aligned}
$$

and are constrained by the Casimir invariants

$$
\begin{aligned}
C_{1} & =\int d x \rho, \quad C_{2}=\int d x E \\
C_{3} & =\int d x\left(\mathrm{v}+\frac{\rho}{2} \sum_{k=1}^{N} \frac{a_{k}}{a_{N+1}^{2}}\left(\nu_{N-1}+a_{N+1} \sum_{l=k}^{N-1} \frac{\nu_{l}-\nu_{l-1}}{A_{l}}\right)^{2}\right), \\
C_{4} & =\frac{\partial E}{\partial x}+\rho, \quad C_{i}=\int d x \rho \nu_{i}, \quad 5 \leq i \leq N+3,
\end{aligned}
$$

where $A_{i}=\sum_{k=1}^{i} a_{k}$. In addition to the already encountered constraints associated with $C_{1}, C_{2}$ and $C_{4}$, the generic $N+1$-moment model derived from the water-bag distribution function possesses further conservation laws. The invariants $C_{5}, \cdots, C_{N+3}$ express the conservation of the number of particles in each bag, whereas $C_{3}$ reflects the conservation of a mean generalized velocity (which reduces to the fluid velocity in the single water-bag limit).

Although it was shown that from water-bag distribution functions it is possible to construct Hamiltonian fluid models for an arbitrary number of moments, the corresponding closure relations are not easy to extract, except for the simplest cases, as in the case of the single water-bag. In Ref. [127] a way to represent such closure relations in terms of reduced moments was presented. The closure for the $N$-water-bag model is indeed a relation of the form $S_{N+1}=S_{N+1}\left(S_{2}, \cdots, S_{N}\right)$, which represents an $N$-1-dimensional manifold in $\mathbb{R}^{N}$, parametrized by $\left(\nu_{1}, \cdots, \nu_{N-1}\right)$.

The projections of the edges of such manifold on the $\left(S_{2}, S_{3}, S_{4}\right)$ space correspond to the closures of the three-moment models obtained by combining all the possible pairs of bags 

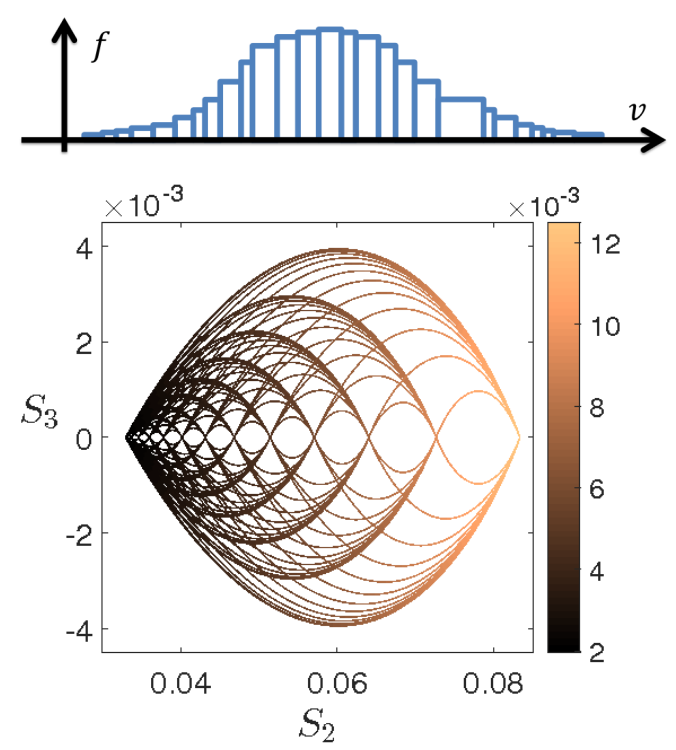

Figure 5. Upper panel: sketch of a bell-shaped water-bag distribution function with twenty-seven bags. Lower panel: projection of the edges of the manifold defining the closure for the distribution function given by the upper panel (after M. Perin, C. Chandre, P.J. Morrison, E. Tassi, Phys. Plasmas, 22, 092309 (2015)).

of the distribution function under consideration. In this way, using the values of $S_{4}$ as a function of $S_{2}$ and $S_{3}$ in order to visualize the edges of the manifolds in the $\left(S_{2}, S_{3}, S_{4}\right)$ space, it is possible to represent all the information related to a given closure relation.

An example for a closure associated with a 27-water-bag distribution function is depicted in Fig. 5.

Finally, we remark that the water-bag closure extends the $N$-delta reductions

$$
f(x, v, t)=\sum_{i=1}^{N} b_{i}(x, t) \delta\left(v-\mathrm{v}_{i}(x, t)\right)
$$

which, in terms of moments, lead to the Zakharov decomposition [135, 138, 139].

$$
P_{n}(x, t)=\sum_{i=1}^{N} b_{i}(x, t) \mathrm{v}_{i}{ }^{n}(x, t),
$$

for non-negative integers $n$. 


\section{B. Hamiltonian closures from drift-kinetic systems}

As mentioned in Sec. IV C, the investigation of low-frequency phenomena such as tokamak turbulence, occurring in the presence of a strong magnetic field, can take advantage of reduced kinetic description such as gyrokinetics, where the evolving distribution functions are defined over a reduced coordinate space, independent of the gyration angle. If, in addition, one is interested in phenomena occurring on scales much larger than the Larmor radius, one can take the limit $k_{\perp} \rho \ll 1$ of the gyrokinetic theory, where $\rho$ is the Larmor radius of the particle of the species under consideration. This leads to a so called drift-kinetic description (see, e.g. Ref. [27]). Similarly to the case of gyrofluid models, fluid models (sometimes also referred to as to drift-fluid models) can be derived from drift-kinetic equations by truncating the infinite hierarchy of equations evolving moments of the drift-kinetic distribution functions.

In this Section, we review recent results concerning closures leading to Hamiltonian driftfluid models derived from Hamiltonian parent drift-kinetic systems.

\section{Two and three-moment drift-fluid models}

In the presence of a uniform and constant magnetic field $\mathbf{B}=B_{0} \hat{z}$, we consider the parent drift-kinetic model in which the evolution equation, is given by

$$
\frac{\partial f}{\partial t}+\frac{c}{B_{0}}[\phi, f]+v_{\|} \frac{\partial f}{\partial z}-\frac{q}{m} \frac{\partial \phi}{\partial z} \frac{\partial f}{\partial v_{\|}}=0
$$

In Eq. (330), $f=f\left(x, y, z, v_{\|}, t\right)$ is the average, with respect to the magnetic moment coordinate $\mu=m v_{\perp}^{2} /\left(2 B_{0}\right)$, of the actual drift-kinetic distribution function $\mathrm{f}\left(x, y, z, v_{\|}, \mu, t\right)$,

i.e. $f\left(x, y, z, v_{\|}, t\right)=\left(2 \pi B_{0} / m\right) \int_{0}^{+\infty} d \mu \mathrm{f}\left(x, y, z, v_{\|}, \mu, t\right)$. The distribution function refers to a population of particles of mass $m$ and charge $q$. Here the symbols $v_{\perp}$ and $v_{\|}$denote the velocity coordinates perpendicular and parallel to the magnetic field, respectively. In Eq. (330), the second term represents the transport by the $\mathbf{E} \times \mathbf{B}$ drift, the third term is the advection along the magnetic field, whereas the last term accounts for the action of the 
electric force. Equation (330) can be obtained as a considerably simplified limit of standard gyrokinetic system such as those of Refs. [16, 24]. In the following, we set $c=B_{0}=m=1$ and $q=-1$. We specialize then, without loss of generality, to the case where the distribution $f$ refers to electrons and we assume, for simplicity, that the normalized electrostatic potential $\phi$ is related to $f$ via Poisson's equation, with ions forming a neutralizing background

$$
\Delta \phi=\int d v_{\|}\left(f-\int d^{3} x f\right)
$$

although the results of Sec. VB 1 hold also in the case of more refined relations [140]. We remark that, with the formulation given in Eq. (331), one assumes that the ion density is equal to $\int d^{3} x d v_{\|} f$, corresponding to the spatial average of the electron density over the domain, so that the distribution function $f$ actually contains information also on the ion population.

Equation (330) admits a Hamiltonian formulation in terms of the dynamical field $f$, defined over the domain of coordinates $\left(x, y, z, v_{\|}\right) \in \mathcal{D}_{\mathbf{x}, \mathbf{v}}=[0,1] \times[0,1] \times[0,1] \times \mathbb{R}$. The Hamiltonian functional corresponds to the sum of parallel kinetic and electrostatic energy and is given by

$$
H(f)=\frac{1}{2} \int d^{3} x d v_{\|} f\left(v_{\|}^{2}-\phi\right)
$$

whereas the Poisson bracket reads

$$
\{F, G\}=\int d^{3} x d v_{\|} f\left(\left[F_{f}, G_{f}\right]+\left[F_{f}, G_{f}\right]_{v}\right)
$$

where

$$
[g, h]_{v}=\frac{\partial g}{\partial z} \frac{\partial h}{\partial v_{\|}}-\frac{\partial g}{\partial v_{\|}} \frac{\partial h}{\partial z} .
$$

In Refs. [140, 141], the Hamiltonian closures for two and three-moment reductions of the system (330) were derived.

The procedure for the derivation of the Hamiltonian closures corresponds essentially to that already described in Secs. VA 1 and VA2. Fluid moments of the drift-kinetic distribution function, with respect to the parallel velocity, are defined as follows:

$$
P_{n}(x, y, z, t)=\int d v_{\|} v_{\|}^{n} f\left(x, y, z, v_{\|}, t\right)
$$


for a non-negative integer $n$.

The Hamiltonian (332) can, as was the case for the Vlasov systems, easily be expressed in terms of the moments as

$$
H\left(P_{0}, P_{2}\right)=\frac{1}{2} \int d^{3} x\left(P_{2}-\phi P_{0}\right)
$$

(note that, from Eq. (331), the electrostatic potential $\phi$ depends on $P_{0}$ as $\Delta \phi=\widetilde{P_{0}}$ ).

Making use of the functional chain rule, the Poisson bracket (333) is rewritten, in terms of the moment variables, as

$$
\{F, G\}=\sum_{m, n=0}^{+\infty} \int d^{3} x\left(P_{m+n}\left[F_{m}, G_{n}\right]+P_{m+n-1}\left(n G_{n} \partial_{z} F_{m}-m F_{m} \partial_{z} G_{n}\right)\right) .
$$

Note that the second term in the bracket (337) corresponds (provided one replaces $z$ with $x$ ) to the bracket (259), derived from the Vlasov equation. The drift-kinetic system adds then a further contribution, corresponding to the term accounting for the advection in the plane perpendicular to the magnetic field. Namely the presence of this term implies an important difference with respect to the previously treated Vlasov cases, with regard to the derivation of Hamiltonian closures. Indeed, unlike for Vlasov systems, evaluating (337) between two functionals $F\left(P_{0}, P_{1}\right)$ and $G\left(P_{0}, P_{1}\right)$ does not yield again a functional of $P_{0}$ and $P_{1}$ but

$$
\begin{aligned}
& \{F, G\}=\int d^{3} x\left(P_{0}\left[F_{0}, G_{0}\right]+P_{1}\left(\left[F_{1}, G_{0}\right]+\left[F_{0}, G_{1}\right]\right)+P_{2}\left[F_{1}, G_{1}\right]\right. \\
& \left.+P_{0}\left(G_{1} \partial_{z} F_{0}-F_{1} \partial_{z} G_{0}\right)+P_{1}\left(G_{1} \partial_{z} F_{1}-F_{1} \partial_{z} G_{1}\right)\right)
\end{aligned}
$$

which depends expicitly on $P_{2}$. For a two-moment model derived from the drift-kinetic system, therefore, Hamiltonian closures must be sought in the form $P_{2}=P_{2}\left(P_{0}, P_{1}\right)$. In Ref. [140] it is shown that (again assuming independence of the closure relation on the derivatives of the moments and on the spatial coordinates) the only Hamiltonian closure is given by

$$
P_{2}=\frac{P_{1}^{2}}{P_{0}}+\mathcal{A} P_{0}^{3}
$$

with constant $\mathcal{A}$. Inserting (339) into Eq. (338) yields then a Poisson bracket.

In terms of pressure $p=P_{2}-P_{1}^{2} / P_{0}$ and mass density $\rho=P_{0}$, the closure (339) reads $p=\mathcal{A} \rho^{3}$, which corresponds to the adiabatic equation of state for an ideal barotropic gas 
composed of molecules with one degree of freedom. Following Sec. V A 3, we observe then that this corresponds to a single water-bag distribution function, with the constant $\mathcal{A}$ related to the height of the bag and, consequently, to the temperature of the electron population.

The resulting model equations can be written as

$$
\begin{aligned}
\frac{\partial \rho}{\partial t}+[\phi, \rho]+\frac{\partial(\rho u)}{\partial z} & =0 \\
\frac{\partial u}{\partial t}+[\phi, u]+\frac{\partial}{\partial z}\left(\frac{u^{2}}{2}-\phi+\frac{3}{2} \mathcal{A} \rho^{2}\right) & =0
\end{aligned}
$$

where we replaced the dynamical variable $P_{1}$ in favor of the parallel fluid velocity $u=P_{1} / P_{0}$. The model (340)-(341) corresponds to a single water-bag version of the model derived in Ref. [131]. Two Casimir invariants constrain the dynamics and are given by

$$
C_{1}=\int d^{3} x \rho, \quad C_{2}=\int d^{3} x u
$$

corresponding to conservation of the total mass and of the mean parallel velocity. These are clearly analogous to the invariants $C_{1}$ and $C_{3}$ in Eq. (313), indicating that, in spite of the differences introduced in the Poisson bracket by the perpendicular dynamics, such Casimir invariants survive when moving from the 1D Vlasov case to the drift-kinetic case in the frame of the single water-bag reduction. We remark that in Ref. [142], the above results were generalized to the case where the drift-kinetic distribution function is given by the sum of a perturbation with a non-uniform Maxwellian, thus leading to a Hamiltonian two-moment extension of the Charney-Hasegawa-Mima equation for drift waves.

In Ref. [141] the above result was extended and the Hamiltonian closures for threemoment drift-fluid models were derived. In this case, the Hamiltonian (332) can be conveniently rewritten as a functional of three fluid dynamical fields, as

$$
H\left(\rho, u, S_{2}\right)=\frac{1}{2} \int d^{3} x\left(\rho u^{2}+\rho^{3} S_{2}-\phi \rho\right),
$$

where, analogously to the Vlasov systems, we introduced the reduced moments with respect to the parallel velocity as

$$
S_{n}(x, y, z, t)=\frac{1}{\rho^{n+1}(x, y, z, t)} \int d v_{\|}\left(v_{\|}-u(x, y, z, t)\right)^{n} f\left(x, y, z, v_{\|}, t\right), \quad \text { for } n \geq 2
$$


We remark that, although the drift-fluid Hamiltonian remains (336), the Jacobi identity implies that constraints on both $P_{3}=P_{3}\left(P_{0}, P_{1}, P_{2}\right)$ and $P_{4}=P_{4}\left(P_{0}, P_{1}, P_{2}\right)$ have to be satisfied. These constraints can be best expressed in terms of the reduced moments. A necessary condition imposed by the Jacobi identity is that $S_{3}=S_{3}\left(S_{2}\right)$. Furthermore, the equations

$$
3 S_{2}-\left(S_{3}^{\prime}\right)^{2}+4 S_{3} S_{3}^{\prime \prime}=0
$$

and

$$
S_{4}=\frac{1}{5}\left(4 S_{3} S_{3}^{\prime}+9 S_{2}^{2}\right)+\frac{\mathcal{C}}{\rho^{5}}
$$

where $\mathcal{C}$ is a constant, have to be satisfied. We note that Eq. (346) indicates that, once a solution is found for $S_{3}$ from Eq. (345), then $S_{4}$ is immediately determined. Moreover, preventing $S_{4}$ (which corresponds to the kurtosis of the underlying distribution function) going to infinity as $\rho$ tends to zero, implies $\mathcal{C}=0$. In Ref. [141], Eq. (345) is investigated after transforming it into an Emden-Fowler (see, e.g. Ref. [143]) form. It is then shown that the only physically plausible solution corresponds to a two-water-bag distribution function of the form

$$
\begin{gathered}
f\left(\mathbf{x}, v_{\|}, t\right)=a_{1}\left[\Theta\left(v_{\|}-u_{1}(\mathbf{x}, t)\right)-\Theta\left(v_{\|}-u_{2}(\mathbf{x}, t)\right)\right] \\
+\left(a_{1}+a_{2}\right)\left[\Theta\left(v_{\|}-u_{2}(\mathbf{x}, t)\right)-\Theta\left(v_{\|}-u_{3}(\mathbf{x}, t)\right)\right]
\end{gathered}
$$

where $a_{1}$ and $a_{1}+a_{2}$ are the heights of the two bags and $u_{1}, u_{2}$ and $u_{3}$ correspond to the contour velocities. The solution for $S_{3}$ can be expressed in parametric form as

$$
\begin{aligned}
& S_{2}\left(n_{1}\right)=\frac{a_{1}^{2}+6 a_{2} a_{1} n_{1}^{2}+4 a_{2}\left(a_{2}-a_{1}\right) n_{1}^{3}-3 a_{2}^{2} n_{1}^{4}}{12 a_{1}^{2}\left(a_{1}+a_{2}\right)^{2}}, \\
& S_{3}\left(n_{1}\right)=-\frac{a_{2}\left(n_{1}-1\right)^{2} n_{1}^{2}\left(a_{1}+a_{2} n_{1}\right)^{2}}{4 a_{1}^{3}\left(a_{1}+a_{2}\right)^{3}},
\end{aligned}
$$

where the parameter $n_{1} \in[0,1]$ corresponds to the density of particles in the first bag. 
Considering this solution for $S_{3}$, the resulting Hamiltonian three-moment model reads

$$
\begin{aligned}
& \frac{\partial \rho}{\partial t}+[\phi, \rho]+\frac{\partial(\rho u)}{\partial z}=0 \\
& \frac{\partial u}{\partial t}+[\phi, u]+u \frac{\partial u}{\partial z}+\frac{1}{\rho} \frac{\partial\left(\rho^{3} S_{2}\right)}{\partial z}-\frac{\partial \phi}{\partial z}=0 \\
& \frac{\partial S_{2}}{\partial t}+\left[\phi, S_{2}\right]+u \frac{\partial S_{2}}{\partial z}+\frac{1}{\rho^{3}} \frac{\partial\left(\rho^{4} S_{3}\right)}{\partial z}=0 .
\end{aligned}
$$

Equation (349) can be reformulated as en evolution equation for the pressure $p=\rho^{3} S_{2}$ as

$$
\frac{\partial p}{\partial t}+[\phi, p]+u \frac{\partial p}{\partial z}+3 p \frac{\partial u}{\partial z}+\frac{\partial\left(\rho^{4} S_{3}\right)}{\partial z}=0
$$

Although with a different, non-Hamiltonian, closure, this three-moment model was studied in Ref. [144].

Casimir invariants for this model are given by

$$
C_{1}=\int d^{3} x \rho, \quad C_{2}=\int d^{3} x\left(u-\frac{1}{4} \rho \kappa_{0}^{2}\right), \quad C_{3}=\int d^{3} x \rho \kappa_{0},
$$

where $\kappa_{0}^{\prime}\left(S_{2}\right)=1 / \sqrt{2 S_{3}}$. Note the analogy with the Casimir invariants (280). Indeed, a formal similarity exists between Eqs. (347), (348), (350) and the three-moment model given by Eqs. (271), (272) and (274). Analogous terms are present in both models, and the differences arise due to the presence of a perpendicular dynamics, associated with the dependence of the moments on two additional space coordinates, and to the closures. In particular, we remark that in the model derived from the Vlasov system, a family of Hamiltonian closures exists, which one can rewrite as $S_{3}=\overline{\mathcal{Q}}\left(S_{2}\right)$, with arbitrary $\overline{\mathcal{Q}}$. In the drift-fluid model, on the other hand, the dependence of $S_{3}$ on $S_{2}$ leading to a Hamiltonian closure is not arbitrary. The introduction of the perpendicular dynamics, associated with the first contribution on the right-hand side of Eq. (337), imposes stronger constraints on the drift-fluid Hamiltonian closures than in the Vlasov case.

\section{Reduced drift-fluid models derived in the $\delta f$ approximation}

For plasmas close to equilibrium and in the presence of a strong guide field, the driftkinetic description can be further simplified by adopting the so called $\delta f$ approximation, 
which we assume in the following. Simplifications introduced by this approximation allow us to consider, with respect to the electrostatic case of Sec. VB 1, a more general magnetic field $\mathbf{B}(x, y, z, t)=B_{0} \hat{z}+\nabla \psi(x, y, z, t) \times \hat{z}$.

Considering, again, without loss of generality, the case of the electron population, in the $\delta f$ approximation one decomposes the drift-kinetic distribution function $\mathrm{f}$, in dimensional units, as

$$
\mathrm{f}\left(x, y, z, v_{\|}, \mu, t\right)=\mathrm{F}_{e q}\left(v_{\|}, \mu\right)+\tilde{\mathbf{f}}\left(x, y, z, v_{\|}, \mu, t\right),
$$

where $\mathrm{F}_{e q}\left(v_{\|}, \mu\right)=n_{0}\left(2 \pi T_{e} / m_{e}\right)^{-3 / 2} \exp \left(-m_{e} v_{\|}^{2} / 2 T_{e}-\mu B_{0} / T_{e}\right)$ is a Maxwellian equilibrium distribution function, characterized by uniform temperature $T_{e}$ and density $n_{0}$. The function $\tilde{\mathrm{f}}$, on the other hand, indicates a space and time-dependent perturbation. Denoting by $\tau$ and $L$ characteristic time and perpendicular length scales of the fluctuations, the following ordering is then assumed:

$$
\begin{aligned}
& \tau \partial_{t} \sim L \partial_{z} \sim \frac{\tilde{\mathrm{f}}}{\mathrm{F}_{e q}} \sim \frac{|\nabla \psi|}{B_{0}} \sim \frac{|\nabla \phi|}{B_{0}} \sim \epsilon \ll 1, \\
& L \partial_{x} \sim L \partial_{y} \sim \frac{\tau}{L} v_{\|} \sim 1 .
\end{aligned}
$$

Note the similarity between the orderings (352) and (130).

From the electromagnetic gyrokinetic equations (in the drift-kinetic limit $k_{\perp} \rho \ll 1$ ), by retaining only terms of order $\epsilon^{2}$ according to the ordering (352), it is possible to derive the following $\delta f$ drift-kinetic equation

$$
\frac{\partial g}{\partial t}+\frac{c}{B}\left[\phi-\frac{v_{\|}}{c} \psi, g\right]+v_{\|} \frac{\partial}{\partial z}\left(g-e \frac{\mathcal{F}_{e q}}{T_{e}}\left(\phi-\frac{v_{\|}}{c} \psi\right)\right)=0 .
$$

In Eq. (353), we introduced the field variable

$$
g\left(x, y, z, v_{\|}, t\right)=\tilde{f}\left(x, y, z, v_{\|}, t\right)-\frac{e}{T_{e}} \frac{v_{\|}}{c} \mathcal{F}_{e q}\left(v_{\|}\right) \psi(x, y, z, t),
$$

where $\tilde{f}=\left(2 \pi B_{0} / m_{e}\right) \int_{0}^{+\infty} d \mu \tilde{\mathrm{f}}$ and

$$
\mathcal{F}_{e q}=\frac{2 \pi B_{0}}{m_{e}} \int_{0}^{+\infty} d \mu \tilde{\mathrm{F}}_{e q}=n_{0}\left(\frac{m_{e}}{2 \pi T_{e}}\right)^{1 / 2} \exp \left(-m_{e} v_{\|}^{2} /\left(2 T_{e}\right)\right)
$$

are the averages of the perturbation and equilibrium distribution functions with respect to the magnetic moment. The modified distribution function $g$, with the second term of Eq. 
(354), accounts also for a linearized form of the term responsible for the perturbation of the magnetic field. Equation (354) can be obtained as a simplified limit of the $\delta f$ gyrokinetic equation adopted, for instance, in Ref. [24]. We remark that the possibility of making use of a field variable averaged over the magnetic moment, depends on the fact that we are neglecting curvature and inhomogeneity in the background magnetic field, which would otherwise introduce an explicit dependence on the magnetic moment, of the coefficients of some terms in the drift-kinetic equation.

We suppose, for simplicity, that the electrostatic and magnetic potential are related to the modified distribution function by

$$
\begin{aligned}
& \left(\Gamma_{0}-1\right) \frac{e \phi}{T_{i}}=\frac{1}{n_{0}} \int d v_{\|} g, \\
& \Delta_{\perp} \psi=\frac{4 \pi e^{2} n_{0}}{m_{e} c^{2}} \psi+\frac{4 \pi e}{c} \int d v_{\|} v_{\|} g .
\end{aligned}
$$

Equation (356) corresponds to the quasi-neutrality relation balancing the electron density perturbations and the polarization of ions fluctuating around a Maxwellian distribution with temperature $T_{i}$, whereas Eq. (357) is the parallel component of Ampère's law where the parallel ion velocity has been neglected. Ion dynamics, which we do not include here while irrelevant for the sake of describing the Hamiltonian closure, can be specified providing relations between ion (kinetic or fluid) field variables and the electromagnetic potentials. We remark that, for instance, the results presented in Sec. VB 2 can easily be extended to include an ion dynamics governed by isothermal gyrofluid equations [145].

The $\delta f$ drift-kinetic equation (353), completed by the relations (356)-(357) admits a Hamiltonian formulation with $g$ as field variable defined over $\mathcal{D}_{\mathbf{x}, \mathbf{v}}=[0,1] \times[0,1] \times[0,1] \times \mathbb{R}$. The Hamiltonian functional is given by

$$
H(g)=\frac{1}{2} \int d^{3} x d v_{\|}\left(\frac{T_{e}}{\mathcal{F}_{e q}} g^{2}-e\left(\phi-\frac{v_{\|}}{c} \psi\right) g\right),
$$

and the Poisson bracket reads

$$
\{F, G\}=\int d^{3} x d v_{\|}\left(\frac{c}{e B_{0}} g\left[F_{g}, G_{g}\right]-v_{\|} \frac{\mathcal{F}_{e q}}{T_{e}} F_{g} \frac{\partial G_{g}}{\partial z}\right) .
$$


From the Hamiltonian drift-kinetic system under consideration, it is possible to derive a hierarchy of fluid equations that can be conveniently expressed in terms of Hermite moments with respect to the parallel velocity $v_{\|}$normalized to the electron thermal speed $v_{t e}=$ $\left(T_{e} / m_{e}\right)^{1 / 2}$. We define the Hermite moment of order $n$ as

$$
g_{n}(x, y, z, t)=\frac{1}{n_{0} \sqrt{n !}} \int d v_{\|} H_{n}\left(v_{\|} / v_{t e}\right) g\left(x, y, z, v_{\|}, t\right)
$$

where $H_{n}$ denotes the $n$-th Hermite polynomial.

Although we make use here of Hermite moments, ordinary moments $P_{n}=\int d v_{\|} v_{\|}^{n} g$ can be obtained as linear combinations of the Hermite moments. In particular, we recall that the first Hermite polynomials are given by $H_{0}\left(v_{\|} / v_{t e}\right)=1, H_{1}\left(v_{\|} / v_{t e}\right)=v_{\|} / v_{t e}, H_{2}\left(v_{\|} / v_{t e}\right)=$ $\left(v_{\|} / v_{t e}\right)^{2}-1, H_{3}\left(v_{\|} / v_{t e}\right)=\left(v_{\|} / v_{t e}\right)^{3}-3\left(v_{\|} / v_{t e}\right)$. The first Hermite moments possess then a clear physical meaning as they can be written as

$$
\begin{aligned}
& g_{0}=\frac{n}{n_{0}}, \quad g_{1}=\frac{u}{v_{t e}}-\frac{e}{m_{e} v_{t e} c} \psi, \\
& g_{2}=\frac{t_{\|}}{\sqrt{2} m_{e} v_{t e}^{2}}, \quad g_{3}=\sqrt{\frac{2}{3}} \frac{q_{\|}}{n_{0} T_{e} v_{t e}},
\end{aligned}
$$

where $n, u, t_{\|}$and $q_{\|}$indicate the (dimensional) fluctuations for the electron particle density, parallel fluid velocity, temperature and heat flux, respectively.

In order to express the hierarchy of fluid equations in terms of Hermite moments, we observe first that the quasi-neutrality relation (356) and Ampère's law (357) can easily be rewritten as

$$
\begin{aligned}
& \left(\Gamma_{0}-1\right) \frac{e \phi}{T_{i}}=g_{0}, \\
& \Delta_{\perp} \psi=\frac{4 \pi e^{2} n_{0}}{m_{e} c^{2}} \psi+\frac{4 \pi e n_{0} v_{t e}}{c} g_{1} .
\end{aligned}
$$

From Eq. (353) it can then be shown that the evolution equation for the generic Hermite moment of order $n$ is:

$$
\begin{aligned}
& \frac{\partial g_{n}}{\partial t}=-\frac{c}{B_{0}}\left[\phi, g_{n}\right]+\sqrt{n+1} \frac{v_{t e}}{B_{0}}\left[\psi, g_{n+1}\right]+\sqrt{n} \frac{v_{t e}}{B_{0}}\left[\psi, g_{n-1}\right] \\
& -\sqrt{n+1} v_{t e} \frac{\partial}{\partial z} g_{n+1}-\sqrt{n} v_{t e} \frac{\partial}{\partial z} g_{n-1} \\
& +\delta_{n 1} v_{t e} \frac{\partial}{\partial z} \frac{e \phi}{T_{e}}-\sqrt{n !} v_{t e}^{2}\left(\delta_{n 0}+\delta_{n 2}\right) \frac{\partial}{\partial z} \frac{e \psi}{c T_{e}} .
\end{aligned}
$$


The evolution of $g_{n}$ requires knowing the evolution of $g_{n+1}$, which in turn depends on the evolution of $g_{n+2}$ and so on, thus leading to a closure problem. In Ref. [145] it is shown that the closure relation

$$
g_{N+1}=\alpha g_{N}
$$

with constant $\alpha$ and positive integer $N$, leads to a Hamiltonian $N+1$-moment drift-fluid model, for arbitrary $N$.

The Hamiltonian of the resulting drift-fluid model can be derived from the parent Hamiltonian (358) by replacing $g$ with its truncated expansion in terms of Hermite polynomials

$$
g\left(x, y, z, v_{\|}, t\right)=\sum_{n=0}^{N} \frac{g_{n}(x, y, z, t)}{\sqrt{n !}} H_{n}\left(\frac{v_{\|}}{v_{t e}}\right) \mathcal{F}_{e q}\left(v_{\|}\right),
$$

and making use of the orthogonality relation between Hermite polynomials. The resulting drift-fluid Hamiltonian, adopting $\left(g_{0}, \cdots, g_{N}\right)$ as dynamical field variables, reads

$$
H\left(g_{0}, \cdots, g_{N}\right)=\frac{n_{0} T_{e}}{2} \sum_{n=0}^{N} \int d^{3} x\left(g_{n}^{2}-\frac{e \phi}{T_{e}} g_{0}+\frac{e v_{t e}}{T_{e} c} \psi g_{1}\right)
$$

The Poisson bracket, on the other hand, is best expressed in terms of the variables $G_{i}=$ $U_{i m}^{T} g_{m}$, for $i=0, \cdots, N$. In this expression, $U^{T}$ indicates the transpose of the orthogonal matrix $U$ such that $U^{T} W U=\Lambda$, where $\Lambda=\operatorname{diag}\left(\lambda_{0}, \lambda_{1}, \cdots, \lambda_{N}\right)$ with $\lambda_{0}, \cdots, \lambda_{N}$ indicating the eigenvalues of the symmetric matrix

$$
W=\left(\begin{array}{cccccc}
0 & 1 & 0 & 0 & \ldots & 0 \\
1 & 0 & \sqrt{2} & 0 & \ldots & 0 \\
0 & \sqrt{2} & 0 & \sqrt{3} & \ldots & 0 \\
0 & 0 & \sqrt{3} & 0 & \ldots & 0 \\
& \ldots & & & \ldots & \\
& \ldots & & & \ldots & \\
0 & 0 & 0 & \ldots & 0 & \sqrt{N} \\
0 & 0 & 0 & \ldots & \sqrt{N} & \alpha \sqrt{N+1}
\end{array}\right)
$$

Note that, once the fluid hierarchy is closed by imposing the relation (366), the evolution equation for the generic moment $g_{n}$ can be written, with the help of the elements $W_{n i}$ of the 
matrix $W$, as

$$
\begin{aligned}
& \frac{\partial g_{n}}{\partial t}=-\frac{c}{B_{0}}\left[\phi, g_{n}\right]+\frac{v_{t e}}{B_{0}}\left[\psi, W_{n i} g_{i}\right]-v_{t e} \frac{\partial}{\partial z} W_{n i} g_{i}+\delta_{n 1} v_{t e} \frac{\partial}{\partial z} \frac{e \phi}{T_{e}} \\
& -\sqrt{n !}\left(\delta_{n 0}+\delta_{n 2}\right) v_{t e}^{2} \frac{\partial}{\partial z} \frac{e \psi}{c T_{e}}, \quad 0 \leq n \leq N .
\end{aligned}
$$

The transformation $U^{T}$ is namely the one that maps the original field variables $\left(g_{0}, \cdots, g_{N}\right)$ into the corresponding normal fields $\left(G_{0}, \cdots, G_{N}\right)$, in terms of which, as already seen in many previous cases, the Poisson bracket reduces into its simplest form. In the specific case, the Poisson bracket is given by

$$
\{F, G\}=\sum_{i=0}^{N} \frac{c}{e B_{0} n_{0} v_{(i)}} \int d^{3} x G_{i}\left[F_{G_{i}}, G_{G_{i}}\right]+\sum_{i=0}^{N} \frac{v_{t e}}{T_{e} n_{0}} \lambda_{i} \int d^{3} x F_{G_{i}} \frac{\partial G_{G_{i}}}{\partial z},
$$

where $v_{(0)}, \cdots, v_{(N)}$ are constants determined by the normalization of the eigenvectors of $W$.

The model equations (370), when expressed in terms of the normal fields, correspond to

$$
\begin{aligned}
& \frac{\partial G_{i}}{\partial t}+\frac{c}{B_{0}}\left[\phi-\lambda_{i} \frac{v_{t e}}{c} \psi, G_{i}\right]+v_{t e} \lambda_{i} \frac{\partial G_{i}}{\partial z}-v_{t e} \lambda_{i} v_{(i)} \frac{\partial}{\partial z} \frac{e \phi}{T_{e}} \\
& +\frac{v_{t e}}{c} \lambda_{i}^{2} v_{(i)} \frac{\partial}{\partial z} \frac{e \psi}{T_{e}}=0, \quad i=0, \cdots, N .
\end{aligned}
$$

In particular note that, in the $2 \mathrm{D}$ limit, Eqs. (372) reduce to equations expressing, for $i=$ $0, \cdots, N$, the advection of the normal fields $G_{i}$ by means of stream functions $\phi-\lambda_{i}\left(v_{t e} / c\right) \psi$, which are reminiscent of the $\mathbf{E} \times \mathbf{B}$ advection and of the free streaming along the magnetic field.

As a simple example illustrating one member of the infinite family of Hamiltonian driftfluid models that can be derived in this way, we consider the case $N=1$ and $\alpha=0$. Upon introducing the normalized quantities

$$
\begin{array}{r}
t=\frac{v_{A}}{L} \hat{t}, \quad x=\frac{\hat{x}}{L}, \quad y=\frac{\hat{y}}{L}, \quad z=\frac{\hat{z}}{L}, \quad d_{i, e}=\frac{\hat{d}_{i, e}}{L}, \quad \rho_{s, i}=\frac{\hat{\rho}_{s, i}}{L}, \\
n_{e}=\frac{L}{\hat{d}_{i}} \frac{\hat{n}}{n_{0}}, \quad u_{e}=\frac{L}{\hat{d}_{i}} \frac{\hat{u}}{v_{A}}, \quad \psi=\frac{\hat{\psi}}{B_{0} L}, \quad \phi=c \frac{\hat{\phi}}{B_{0} L v_{A}},
\end{array}
$$

where the carets denote the dimensional quantities, the two-field model equations for the case $N=1, \alpha=0$ can be written as

$$
\begin{aligned}
& \frac{\partial n_{e}}{\partial t}+\left[\phi, n_{e}\right]+\nabla_{\|} u_{e}=0, \\
& \frac{\partial \psi_{e}}{\partial t}+\left[\phi, \psi_{e}\right]-\rho_{s}^{2} \nabla_{\|} n_{e}+\frac{\partial \phi}{\partial z}=0,
\end{aligned}
$$


complemented by the relations

$$
\begin{aligned}
& n_{e}=\frac{\Gamma_{0}-1}{\rho_{i}^{2}} \phi, \\
& \Delta_{\perp} \psi=u_{e} .
\end{aligned}
$$

For $N=1$, the choice $\alpha=0$ corresponds to the closure $g_{2}=0$ which, according to Eq. (361), means isothermal electrons. The model (374)-(377) describes the dynamics of isothermal electron density fluctuations coupled to that of a magnetic field allowed to reconnect because of electron inertia. Eqs. (374)-(375) correspond indeed to Eqs.(143)-(144) (upon identifying $\omega$ with $\left.n_{e}\right)$ and to Eqs. (205)-(206) in the limit $\kappa=0$, i.e. neglecting background magnetic curvature. The Hamiltonian of the model, given by Eq. (368), corresponds then to

$$
\begin{aligned}
& H\left(\psi_{e}, n_{e}\right)=\frac{1}{2} \int d^{3} x\left(\rho_{s}^{2} n_{e}^{2}+d_{e}^{2} u_{e}^{2}-\phi n_{e}-\psi u_{e}\right) \\
& =\frac{1}{2} \int d^{3} x\left(\rho_{s}^{2} n_{e}^{2}+\left|\nabla_{\perp} \psi\right|^{2}+d_{e}^{2}\left|\Delta_{\perp} \psi\right|^{2}-\phi n_{e}\right),
\end{aligned}
$$

which coincides with Eq. (145), if $n_{e}$ is replaced with $\omega$ and if the relation between $\phi$ and $n_{e}$ is given by $n_{e}=\Delta_{\perp} \phi$ (which corresponds indeed to the cold-ion limit of Eq. (376)). With the analogous prescription, one obtains that the Poisson bracket corresponds to

$$
\begin{aligned}
& \{F, G\}=\int d^{3} x\left(\psi_{e}\left(\left[F_{\psi_{e}}, G_{n_{e}}\right]+\left[F_{n_{e}}, G_{\psi_{e}}\right]\right)+n_{e}\left(d_{e}^{2} \rho_{s}^{2}\left[F_{\psi_{e}}, G_{\psi_{e}}\right]+\left[F_{n_{e}}, G_{n_{e}}\right]\right)\right. \\
& \left.+F_{n_{e}} \frac{\partial G_{\psi_{e}}}{\partial z}+F_{\psi_{e}} \frac{\partial G_{n_{e}}}{\partial z}\right)
\end{aligned}
$$

which can be compared with the bracket (146). Furthermore, the Poisson bracket (379) can be written in the simplified form (371) in terms of the normal fields

$$
G_{ \pm}=\psi_{e} \pm d_{e} \rho_{s} n_{e}
$$

associated with the Casimir invariants $C_{1}=\int d^{3} x G_{+}, C_{2}=\int d^{3} x G_{-}$.

As a second example, consider the case $N=3, \alpha=0$, where in the $2 \mathrm{D}$ limit one obtains the four evolution equations (229)-(232).

Therefore, we have shown that, from the infinite family of Hamiltonian fluid models derived from the drift-kinetic equations, it is possible to retrieve reduced drift-fluid models 
known in the literature. Their Hamiltonian structure, which was obtained a posteriori, in the original derivation, can then be put in correspondance with the Hamiltonian structure of the parent drift-kinetic model. More in general, given the Hamiltonian structure of a reduced drift-fluid model, if the Hamiltonian is of the form (358) and the Poisson bracket in terms of the normal fields is of the form (371), it is then possible to conclude that the model admits a derivation from a drift-kinetic model, with a closure of the form $g_{N+1}=\alpha g_{N}$.

In Ref. [32] it was shown that, by a similar argument, it is possible to derive, from a drift-kinetic model, a Hamiltonian drift-fluid model evolving six field variables for each species, including perpendicular temperature and heat flux fluctuations, so that also fluid anisotropy effects can be modelled in the Hamiltonian context.

\section{CONCLUSIONS}

The derivation and the analysis of Hamiltonian structures of fluid models for plasmas, characterized by different closures, is a subject which is attracting a considerable interest not only in the plasma physics community but also among applied mathematicians interested in geometrical mechanics and dynamical systems. The Hamiltonian approach to the derivation of fluid models provides a complementary viewpoint with respect to more traditional approaches to the closure problem adopted in plasma physics, such as, for instance, those mentioned in Sec. I. Recent results based on this approach have been described in this review, trying also to show how the Hamiltonian approach provides a unifying framework for various fluid models. In Sec. III the classical results on the Hamiltonian formulation for adiabatic and double-adiabatic MHD were recalled and we showed a recent application of Dirac's theory of constraints which made it possible to impose the incompressibility closure to the MHD system, while preserving a Hamiltonian structure. In perspective, we think that Dirac's theory might have other applications in this context, regarding, for instance, imposing constraints such as quasi-neutrality or pressure balance, without losing control of the Hamiltonian structure. In Sec. III, it was also shown how the Hamiltonian character of 
barotropic MHD survives the inclusion of important two-fluid effects leading to the Hamiltonian extended MHD model. This naturally opens the way to a number of investigations, for instance on equilibria and stability, inspired from previous results on MHD (see, e.g. Ref. [41-45]). A novel application of the Hamiltonian structure for this model to plasma turbulence, has already been presented in Ref. [146].

The succesful application of reduced fluid models for both laboratory and astrophysical plasmas motivated the search for Hamiltonian structures also for such kind of models. In Sec. IV, after recalling results on classical reduced models, we focused on the recent FFEI model for magnetic reconnection and on gyrofluid models. Hamiltonian reduced models share a common form for the Hamiltonian functional and the Poisson bracket. In particular, they offer a good application of the concept of normal fields, which correspond to dynamical field variables, associated with the Casimir invariants of the Poisson bracket, in terms of which, as we saw, the model equations take remarkably simple forms. An example of the role of such normal fields emerged when we considered, for instance, the six-field gyrofluid model for magnetic reconnection of Sec. IV C 2. The influence of electron heat flux on a 2D magnetic reconnection process was discussed in comparison with the adiabatic case. In both cases, although the magnetic frozen-in condition is violated by electron inertia, alternative topological conservation laws hold. Such conservation laws are associated with the normal fields which, for both considered closures, are Lagrangian invariants.

The potential for future applications of the Hamiltonian approach to reduced fluid models is likely to be important in particular for $2 \mathrm{D}$ geometries, which can be of interest, e.g., for tokamaks and the solar corona. The translational symmetry along one direction, as shown in a number of examples in this review, is associated with infinite families of Casimir invariants. Full advantage from this richness of invariants can be taken for stability investigations by means of the Energy-Casimir method. The formulation of 2D models in terms of normal fields, can also be beneficial for adopting numerical schemes, such as finite volume schemes, suited for advection equations with incompressible velocity fields.

In Sec. V we reviewed recent results on the derivations of Hamiltonian closures based on 
the Hamiltonian structure of the parent kinetic models. This provides a systematic approach to the derivation of Hamiltonian fluid models and avoids the "a posteriori" search for such structure, which can be cumbersome and is often based on a certain amount of intuition. Also, we saw that the approach of deriving the Hamiltonian closures let emerge interesting connections with previosuly derived models. For instance, drift-fluid models with the waterbag closure were retrieved by means of the procedure described in Sec. VB 1 and found to be the only Hamiltonian models within a certain class of closures. Also, some of the reduced fluid models treated in Sec. IV, and originally derived from a two-fluid description of the plasma, were shown to admit a drift-kinetic derivation in the $\delta f$ approximation and with the Hamiltonian closure presented in Sec. VB 2. In this context, it would be desirable to obtain Hamiltonian closures for moments taken with respect to more than one velocity coordinate. In spite of the results of Refs. [32, 56], indeed, little is known about Hamiltonian structures for models accounting for pressure or heat flux anisotropies. As discussed in Ref. [147], there can be energy conserving models with pressure anisotropy, but only for specific closures on the heat fluxes and energy-weighted pressure tensors, such models were shown to possess a Hamiltonian structure. The gyrofluid reduced model with anisotropic pressure and heat flux fluctuations of Ref. [24] is also energy conserving, but the existence of its Hamiltonian structure remains an open question. If it exists, it would be interesting to see how the normal fields compare with those of the isotropic gyrofluid models discussed in Sec. IV C. Moreover, most of the Hamiltonian reduced fluid models known so far, are valid in the low- $\beta$ regime, where magnetic perturbations parallel to the strong guide field can be neglected. In some astrophysical plasmas, as for instance at sub-ion Larmor radius scales in the solar wind [148], such perturbations are, however, non negligible, and temperature anisotropies are important. Finite $\beta$ Hamiltonian models with closures more sophisticated than that adopted for the FFEI model would then be desirable.

A further possible field, where we think that the derivation of Hamiltonian closures might be of interest, lies outside the domain of classical plasmas and concerns the so called quantum plasmas. This field attracted, over the last years, increasing attention for its 
possible applications for the description of, e.g., electron gases in metals, dense matter in the core of giant planets or stars and high density ionized plasmas produced by laser-matter interaction $[149,150]$. Recently, a number of models based on a fluid description of quantum plasmas were proposed and a debate about the regime of validity of such models followed (see, e.g. Ref. [151]). Clearly, the identification of a Hamiltonian structure in a fluid model cannot, by itself, determine about the physical soundness of the model. However, for the same reasons for which we believe the Hamiltonian approach is beneficial for classical plasma fluid models, we think that, in addition to the fundamental investigations about the regimes of validity, the derivation of Hamiltonian closures for quantum hydrodynamic models, might help in identifiying models failing in preserving the original Hamiltonian dynamics. Recent results in this direction, accounting also for dissipative effects in the metriplectic formulation, are presented in Ref. [152].

We find it also important to mention that the identification of Hamiltonian structures opens to the possibility of applying structure-preserving algorithms in numerical simulations. The development of algorithms that preserve geometric features of ordinary or partial differential equations is now a well developed field. However, the applications to continuum plasma physics models is relatively new. In particular, the development of algorithms based on the noncanonical Hamiltonian structure of continuum models is still at an initial stage [153] and possess a considerable potential for applications, including, among others, all the models treated in this review article.

Clearly, the ultimate purpose of the activity on Hamiltonian closures in fluid models for plasmas should be to identify models that provide a better description of experimental results or observations. In this respect, it could be interesting to apply a derivation or identification of Hamiltonian structures to models more directed toward the comparison with experiments. Indeed, to the best of our knowledge, most of the results obtained so far, including those described in this review, concerned relatively simple fluid models in idealized geometries, admitting a restricted class of boundary conditions. It would be interesting to investigate, from the point of view of the Hamiltonian structure, for instance fluid models adopted to 
investigate tokamak turbulence in realistic geometries. Indeed, just from the discussion [33] on the simplified gyrofluid model of Sec. IV C 1, it emerged how the modelling of geometric effects due to magnetic curvature can affect the Hamiltonian structure.

Still in the framework of laboratory plasmas, experiments on magnetic reconnection motivated the search for anisotropic closures for fluid models in weakly collisional plasmas [154]. It might be of interest trying to identify Hamiltonian closures for such fluid models, where, also, some dissipative effects, such as those due to collisions, are weak.

Collisionless space plasmas, finally, could also offer a further field of applications. For instance, in Ref. [147], a family of reduced fluid models for finite- $\beta$, collisionless plasmas,

was derived, with the closure relation on heat flux and energy-weighted pressure tensors left unspecified. Depending on the closure, it was possible to identify Hamiltonian models but also energy-conserving models for which the Hamiltonian structure did not exist (at least in the form of Eqs. (131)-(132)). It would be interesting to compare energy-conserving vs. Hamiltonian models in terms of observables such as, for instance, the turbulent spectrum, and see how the Hamiltonian character affects the comparison with space plasma observations.

\section{ACKNOWLEDGMENTS}

The author wishes to thank Phil Morrison, Maxime Perin, Cesare Tronci and François Waelbroeck for helpful comments on the manuscript.

\section{Appendix A: Jacobi identity for the Poisson bracket of a barotropic fluid}

We intend to show that the Poisson bracket (48) satisfies the Jacobi identity. Upon writing

$$
\begin{aligned}
& \{F, G\}=-\int_{\mathcal{D}_{\mathbf{x}}} d^{3} x\left(\rho\left(F_{\mathbf{M}} \cdot \nabla G_{\rho}-G_{\mathbf{M}} \cdot \nabla F_{\rho}\right)+\mathbf{M} \cdot\left(F_{\mathbf{M}} \cdot \nabla G_{\mathbf{M}}-G_{\mathbf{M}} \cdot \nabla F_{\mathbf{M}}\right)\right) \\
& =\left\langle\frac{\delta F}{\delta \chi} \mid J \frac{\delta G}{\delta \chi}\right\rangle,
\end{aligned}
$$


where $\chi=(\rho, \mathbf{M})$, we can identify the Poisson operator as

$$
J=-\left(\begin{array}{cc}
0 & \nabla \cdot(\rho(\cdot)) \\
\rho \nabla(\cdot) & \nabla(\cdot) \cdot \mathbf{M}+\nabla \cdot(\mathbf{M}(\cdot))
\end{array}\right)
$$

with $(\cdot)$ indicating the place of the function that the operator $J$ acts upon. As discussed in Sec. II, by virtue of the Lemma of Ref. [56], when evaluating functional derivatives of a Poisson bracket in the Jacobi identity, only the contributions due to the dependence of $J$ on the field variables count. Consequently we write

$$
\frac{\delta\{G, H\}}{\delta \rho}=-G_{\mathbf{M}} \cdot \nabla H_{\rho}+H_{\mathbf{M}} \cdot \nabla G_{\rho}+\cdots, \quad \frac{\delta\{G, H\}}{\delta \mathbf{M}}=-\left(G_{\mathbf{M}} \cdot \nabla\right) H_{\mathbf{M}}+\left(H_{\mathbf{M}} \cdot \nabla\right) G_{\mathbf{M}}+\cdots
$$

where the dots indicate the terms that, by virtue of the Lemma of Ref. [56], do not contribute in the Jacobi identity. Then, making use of Eq. (A3) expressed in coordinates, one obtains

$$
\begin{aligned}
& \{F,\{G, H\}\}+\circlearrowleft \\
& =-\int_{\mathcal{D}_{\mathbf{x}}} d^{3} x\left(\rho\left(F_{M_{i}} \partial_{i}\left(-G_{M_{j}} \partial_{j} H_{\rho}+H_{M_{j}} \partial_{j} G_{\rho}\right)+G_{M_{i}} \partial_{i}\left(H_{M_{j}} \partial_{j} F_{\rho}\right)-H_{M_{i}} \partial_{i}\left(G_{M_{j}} \partial_{j} F_{\rho}\right)\right)\right. \\
& \left.+M_{k}\left(F_{M_{i}} \partial_{i}\left(-G_{M_{j}} \partial_{j} H_{M_{k}}+H_{M_{j}} \partial_{j} G_{M_{k}}\right)+\left(G_{M_{j}} \partial_{j} H_{M_{i}}-H_{M_{j}} \partial_{j} G_{M_{i}}\right) \partial F_{M_{k}}\right)\right)+\circlearrowleft=0,
\end{aligned}
$$

where we indicated with the symbol $\circlearrowleft$, the additional terms that one obtains from cyclic permutation of $F, G$ and $H$. It has been then shown that the Poisson bracket (48) (and, consequently the Poisson bracket (41)) satisfies the Jacobi identity.

[1] S. Chapman and T. G. Cowling, The Mathematical Theory of Non-Uniform Gases (Cambridge University Press, 1952).

[2] H. Grad, Commun. Pure and Appl. Math. 2, 311 (1949).

[3] S. I. Braginskii, Zh. Eksp. Teor. Fiz. 33, 459 (1957).

[4] S. I. Braginskii, "Transport processes in a plasma," in Reviews of Plasma Physics, edited by M. A. Leontovich (Consultants Bureau, New York, 1965).

[5] R. Balescu, Transport Processes in Plasmas. Vol. 1 Classical Transport (North-Holland, 1988). 
[6] R. D. Hazeltine, M. Kotschenreuther, and P. J. Morrison, Phys. Fluids 28, 2466 (1985).

[7] G. W. Hammett and F. W. Perkins, Phys. Rev. Lett. 64, 3019 (1990).

[8] P. B. Snyder and G. W. Hammett, Phys. Plasmas 4, 3974 (1997).

[9] T. Passot and P. L. Sulem, Phys. Plasmas 11, 5173 (2004).

[10] P. Goswami, T. Passot, and P. L. Sulem, Phys. Plasmas 12, 2109 (2005).

[11] P. L. Sulem and T. Passot, J. Plasma Phys. , 325810103 (2015).

[12] N. Mattor and S. E. Parker, Phys. Rev. Lett. 79, 3419 (1997).

[13] N. Mattor, Phys. Plasmas 5, 1822 (1998).

[14] H. Sugama, T. H. Watanabe, and W. Horton, Phys. Plasmas 8, 2617 (2001).

[15] A. J. Brizard and T. S. Hahm, Rev. Mod. Phys. 79, 421 (2007).

[16] A. Brizard, Phys. Fluids B 4, 1213 (1992).

[17] G. W. Hammett, W. Dorland, and F. W. Perkins, Phys. Fluids B 4, 2052 (1992).

[18] G. W. Hammett, M. A. Beer, W. Dorland, S. C. Cowley, and S. A. Smith, Plasma Phys. Control. Fusion 35, 973 (1993).

[19] W. Dorland and G. W. Hammett, Phys. Fluids B 5, 812 (1993).

[20] M. A. Beer and G. W. Hammett, Phys. Plasmas 3, 4046 (1996).

[21] P. B. Snyder and G. W. Hammett, Phys. Plasmas 8, 3199 (2001).

[22] B. Scott, Phys. Plasmas 7, 1845 (2000).

[23] D. Strintzi, B. Scott, and A. Brizard, Phys. Plasmas 12, 052517 (2005).

[24] B. Scott, Phys. Plasmas 17, 102306 (2010).

[25] J. Madsen, Phys. Plasmas 20, 072301 (2013).

[26] H. R. Strauss, Phys. Fluids 19, 134 (1976).

[27] R. D. Hazeltine and J. D. Meiss, Plasma Confinement (Dover Publications, 2003).

[28] A. Hasegawa and K. Mima, Phys. Fluids 21, 87 (1978).

[29] A. Hasegawa and M. Wakatani, Phys. Rev. Lett. 50, 682 (1983).

[30] T. J. Schep, F. Pegoraro, and B. N. Kuvshinov, Phys. Plasmas 1, 2843 (1994).

[31] R. Fitzpatrick and F. Porcelli, Phys. Plasmas 11, 4713 (2004), erratum: 14, 049902 (2007).

[32] E. Tassi, Theor. and Math. Phys. 188, 1377 (2016).

[33] I. Keramidas Charidakos, F. L. Waelbroeck, and P. J. Morrison, Phys. Plasmas 22, 112113 (2015).

[34] F. L. Waelbroeck, R. D. Hazeltine, and P. J. Morrison, Phys. Plasmas 16, 032109 (2009). 
[35] D. Grasso and E. Tassi, J. Plasma Phys. 81, 495810501 (2015).

[36] G. F. Chew, M. L. Goldberger, and F. E. Low, Proc. Roy. Soc. A236, 112 (1956).

[37] P. J. Morrison, Phys. Plasmas 12, 058102 (2005).

[38] P. J. Morrison, Rev. Mod. Phys. 70, 467 (1998).

[39] J. E. Marsden and T. S. Ratiu, Introduction to Mechanics and Symmetry (Springer-Verlag, Berlin, 2002).

[40] C. Tronci, E. Tassi, E. Camporeale, and P. Morrison, Plasma Phys. Control. Fusion 56, 095008 (2014).

[41] D. D. Holm, J. E. Marsden, T. S. Ratiu, and A. Weinstein, Physics Reports 123, 2 (1985).

[42] T. Andreussi, P. J. Morrison, and F. Pegoraro, Phys. Plasmas 23, 102112 (2016).

[43] T. Andreussi, P. J. Morrison, and F. Pegoraro, Phys. Plasmas 20, 092104 (2013).

[44] T. Andreussi, P. J. Morrison, and F. Pegoraro, Phys. Plasmas 19, 052102 (2012).

[45] T. Andreussi, P. J. Morrison, and F. Pegoraro, Plasma Phys. and Contr. Fusion 52, 055001 (2010).

[46] F. Bouchet and A. Venaille, Phys. Reports 515, 227 (2012).

[47] H. M. Abdelhamid and Z. Yoshida, Phys. Plasmas 23, 022105 (2016).

[48] H. M. Abdelhamid, M. Lingam, and S. M. Mahajan, The Astrophys. J. 829, 87 (2016).

[49] P. J. Morrison and J. M. Greene, Phys. Rev. Lett. 45, 790 (1980).

[50] R. Salmon, Ann. Rev. Fluid Mech. 20, 225 (1988).

[51] J. E. Marsden, T. Ratiu, and A. Weinstein, Trans. Amer. Math. Soc. 281, 147 (1984).

[52] J. E. Marsden, A. Weinstein, T. Ratiu, T. S. Schmid, and R. G. Spencer, Atti Acad. Sci. Torino Cl. Sci. Fis. Math. Natur. 117, 289 (1983).

[53] D. D. Holm, T. Schmah, and C. Stoica, Geometric Mechanics and Symmetry: From Finite to Infinite Dimensions (Oxford University Press, 2009).

[54] E. C. D’Avignon, P. J. Morrison, and M. Lingam, Phys. Plasmas 23, 062101 (2016).

[55] M. Lingam, P. J. Morrison, and E. Tassi, Phys. Lett. A 379, 570 (2015).

[56] P. J. Morrison, in Mathematical Methods in Hydrodynamics and Integrability in Dynamical Systems, American Institute of Physics Conference Proceedings, Vol. 88, edited by M. Tabor and Y. Treve (American Institute of Physics, 1982) pp. 13-45.

[57] J. E. Marsden, T. Ratiu, and A. Weinstein, in Fluids and Plasmas: Geometry and Dynamics, Contemporary Mathematics, Vol. 28, edited by J. E. Marsden (American Mathematical 
Society, 1984) pp. 55-100.

[58] P. J. Morrison, M. Lingam, and R. Acevedo, Phys. Plasmas 21, 082102 (2014).

[59] M. Lingam and P. J. Morrison, Phys. Lett. A 3526, 570 (2014).

[60] I. Keramidas Charidakos, M. Lingam, P. J. Morrison, R. L. White, and A. Wurm, Phys. Plasmas 21, 092118 (2014).

[61] Y. Kawazura, G. Miloshevich, and P. J. Morrison, Phys. Plasmas 24, 022103 (2017).

[62] P. J. Morrison and R. D. Hazeltine, Phys. Fluids 27, 886 (1984).

[63] F. Sahraoui, G. Belmont, and L. Rezeau, Phys. Plasmas 10, 1325 (2003).

[64] Z. Yoshida and E. Hameiri, J. Phys. A: Math. Theor. 46, 335502 (2013).

[65] N. Padhye and P. J. Morrison, Phys. Lett. A 219, 287 (1996).

[66] N. Padhye and P. J. Morrison, Plasma Physics Reports 22, 960 (1996).

[67] R. G. Spencer and A. N. Kaufman, Phys. Rev. A 25, 2437 (1982).

[68] D. D. Holm and B. A. Kupershmidt, Physica D 7, 330 (1983).

[69] D. Biskamp, Magnetic Reconnection in Plasmas (Cambridge University Press, 2000).

[70] C. Chandre, P. J. Morrison, and E. Tassi, Phys. Lett. A 376, 737 (2012).

[71] P. A. M. Dirac, Can. J. Math. 2, 129 (1950).

[72] A. Hanson, T. Regge, and C. Teitelboim, Constrained Hamiltonian systems (Accademia Nazionale dei Lincei, Roma, 1976).

[73] E. C. G. Sudarshan and N. Mukunda, Classical Dynamics: A Modern Perspective (Wiley, New York, 1974).

[74] K. Sundermeyer, Constrained Dynamics (Springer-Verlag, Berlin, 1982).

[75] C. Chandre, E. Tassi, and P. J. Morrison, Phys. Plasmas 17, 042307 (2010).

[76] C. Chandre, L. de Guillebon, A. Back, E. Tassi, and P. J. Morrison, J. Phys. A: Math. Theor. 46, 125203 (2013).

[77] D. D. Holm and B. A. Kupershmidt, Phys. Fluids 29, 3889 (1986).

[78] R. Lüst, Fortschritte der Physik 7, 503 (1959).

[79] K. Kimura and P. J. Morrison, Phys. Plasmas 21, 082101 (2014).

[80] H. M. Abdelhamid, Y. Kawazura, and Z. Yoshida, J. Phys. A: Math. Theor. 48, 235502 (2015).

[81] D. D. Holm, Phys. Fluids 30, 1310 (1987).

[82] Y. Kawazura and E. Hameiri, Phys. Plasmas 19, 082513 (2012). 
[83] M. Lingam, G. Miloshevich, and P. J. Morrison, Phys. Lett. A 380, 2400 (2016).

[84] D. Grasso, E. Tassi, H. M. Abdelhamid, and P. J. Morrison, Phys. Plasmas 24, 012110 (2017).

[85] J. Shiraishi, S. Ohsaki, and Z. Yoshida, Phys. Plasmas 12, 092308 (2005).

[86] M. Lingam, P. J. Morrison, and G. Miloshevich, Phys. Plasmas 22, 072111 (2015).

[87] The total volume will, however still extend along the $z$ direction, in the interval $0 \leq z \leq 1$ to account, for instance, for the existence of the guide field along $z$, but all fields will be independent of the $z$ coordinate. What we refer to as to 2D geometry for the reduced fluid models, is sometimes referred to as to $2 \frac{1}{2} \mathrm{D}$ geometry.

[88] J. L. Thiffeault and P. J. Morrison, Physica D 136, 205 (2000).

[89] E. Tassi, P. J. Morrison, D. Grasso, and F. Pegoraro, Nucl. Fusion 50, 034007 (2010).

[90] B. D. Scott, Phys. Plasmas 14, 102318 (2007).

[91] E. Cafaro, D. Grasso, F. Pegoraro, F. Porcelli, and A. Saluzzi, Phys. Rev. Lett. 80, 4430 (1998).

[92] D. Grasso, F. Califano, F. Pegoraro, and F. Porcelli, Phys. Rev. Lett. 86, 5051 (2001).

[93] D. Grasso, D. Borgogno, and F. Pegoraro, Phys. Plasmas 14, 055703 (2007).

[94] M. Ottaviani and F. Porcelli, Phys. Rev. Lett. 71, 3802 (1993).

[95] D. Del Sarto, F. Califano, and F. Pegoraro, Mod. Phys. Lett. B 20, 931 (2006).

[96] D. Del Sarto, C. Marchetto, F. Pegoraro, and F. Califano, Plasma Phys. and Contr. Fusion 53, 035008 (2011).

[97] D. Grasso, E. Tassi, and F. L. Waelbroeck, Phys. Plasmas 17, 082312 (2010).

[98] L. Comisso, D. Grasso, E. Tassi, and F. L. Waelbroeck, Phys. Plasmas 19, 042103 (2012).

[99] E. Tassi, P. J. Morrison, F. L. Waelbroeck, and D. Grasso, Plasma Phys. Control. Fusion 50, 085014 (2008).

[100] D. Del Sarto, F. Califano, and F. Pegoraro, Phys. Rev. Lett. 91, 235001 (2003).

[101] R. D. Hazeltine, C. T. Hsu, and P. J. Morrison, Phys. Fluids 30, 3204 (1987).

[102] R. Fitzpatrick, Phys. Plasmas 17, 042101 (2010).

[103] P. J. Morrison, E. Tassi, and N. Tronko, Phys. Plasmas 20, 042109 (2013).

[104] P. Morrison, I. Caldas, and H. Tasso, Zeitschrift für Naturforschung 39a, 1023 (1984).

[105] O. Izacard, C. Chandre, E. Tassi, and G. Ciraolo, Phys. Plasmas 18, 062105 (2011). 
[106] F. L. Waelbroeck, P. J. Morrison, and W. Horton, Plasma Phys. and Contr. Fusion 46, 1331 (2004).

[107] D. Dagnelund and V. P. Pavlenko, Phys. Scripta 71, 293 (2005).

[108] A. J. Wootton, B. A. Carreras, H. Matsumoto, K. McGuire, W. A. Peebles, C. P. Ritz, P. W. Terry, and S. J. Zweben, Phys. Fluids B 2, 2879 (1990).

[109] G. G. Howes, "Kinetic turbulence," in Magnetic fields in diffuse media, edited by A. Lazarian, E. M. de Gouveia Dal Pino, and C. Melioli (Springer, Berlin, Heidelberg, 2015).

[110] D. Grasso, F. Califano, F. Pegoraro, and F. Porcelli, Plasma Phys. Rep. 26, 512 (2000).

[111] F. L. Waelbroeck and E. Tassi, Commun. Nonlinear Sci. Numer. Simulat. 17, 2171 (2012).

[112] H. de Blank, Phys. Plasmas 8, 3927 (2001).

[113] A. Zocco and A. Schekochihin, Phys. Plasmas 18, 102309 (2011).

[114] P. J. Morrison, Phys. Lett. A 80A, 383 (1980).

[115] J. E. Marsden and A. Weinstein, Physica D 4, 394 (1982).

[116] M. Perin, C. Chandre, P. J. Morrison, and E. Tassi, Annals of Physics 348, 50 (2014), corrigendum: 370, 139 (2016).

[117] B. Kupershmidt and J. Manin, Funktsional Anal. i Prilozhen 12, 25 (1978).

[118] J. Gibbons, Physica D 3, 503 (1981).

[119] J. Gibbons, D. D. Holm, and C. Tronci, Phys. Lett. A 372, 4184 (2008).

[120] L. de Guillebon and C. Chandre, Phys. Lett. A 376, 3172 (2012).

[121] M. Perin, C. Chandre, P. J. Morrison, and E. Tassi, J. Phys. A: Math. Theor. 48, 275501 (2015), corrigendum: 49, 269501 (2016).

[122] J. C. Gibbings, Dimensional Analysis (Springer, 2011).

[123] Y. Cheng and J. A. Rossmanith, J. Comp. Appl. Math. 262, 384 (2014).

[124] C. Yuan and R. O. Fox, J. Comp. Phys. 230, 8216 (2011).

[125] R. O. Fox, J. Comp. Phys. 228, 7771 (2009).

[126] E. Chalons, D. Kah, and M. Massot, Commun. Math. Sci. 10, 1241 (2012).

[127] M. Perin, C. Chandre, P. J. Morrison, and E. Tassi, Phys. Plasmas 22, 092309 (2015).

[128] K. V. Roberts and H. L. Berk, Phys. Rev. Lett. 19, 297 (1967).

[129] P. Bertrand and M. R. Feix, Phys. Lett. A 28, 1968 (1968).

[130] H. L. Berk, C. E. Nielsen, and K. V. Roberts, Phys. Fluids 13, 980 (1970). 
[131] P. Morel, E. Gravier, N. Besse, R. Klein, A. Ghizzo, and P. Bertrand, Phys. Plasmas 14, 112109 (2007).

[132] P. Morel, E. Gravier, N. Besse, A. Ghizzo, and P. Bertrand, Commun. Nonlinear Sci. Numer. Simulat. 13, 11 (2008).

[133] E. Gravier, R. Klein, P. Morel, N. Besse, and P. Bertrand, Phys. Plasmas 15, 122103 (2008).

[134] E. Gravier and E. Plaut, Phys. Plasmas 20, 042105 (2013).

[135] A. A. Chesnokov and M. V. Pavlov, Acta Appl. Math. 122, 367 (2012).

[136] P. J. Morrison and G. I. Hagstrom, "Continuum Hamiltonian Hopf bifurcation I," in Nonlinear Physical Systems: Spectral Analysis, Stability and Bifurcations, edited by O. Kirillov and D. Pelinovsky (Wiley, New York, 2014).

[137] G. I. Hagstrom and P. J. Morrison, "Continuum Hamiltonian Hopf bifurcation II," in Nonlinear Physical Systems: Spectral Analysis, Stability and Bifurcations, edited by O. Kirillov and D. Pelinovsky (Wiley, New York, 2014).

[138] V. E. Zakharov, Funct. Anal. Appl. 14, 89 (1980).

[139] V. E. Zakharov, Physica D 3, 193 (1981).

[140] E. Tassi, J. Phys. A: Math. Theor. 47, 195501 (2014).

[141] M. Perin, C. Chandre, and E. Tassi, J. Phys. A: Math. Theor. 49, 305501 (2016).

[142] E. Tassi, Eur. Phys. J. D 68, 196 (2014).

[143] A. D. Polyanin and V. F. Zaitsev, Handbook of Exact Solutions For Ordinary Differential Equations (Chapman and Hall/CRC Press, 2003).

[144] Y. Sarazin, G. Dif-Pradalier, D. Zarzoso, X. Garbet, P. Ghendrih, and V. Grandgirard, Plasma Phys. Control. Fusion 51, 115003 (2009).

[145] E. Tassi, Annals of Physics 362, 239 (2015).

[146] G. Miloshevich, M. Lingam, and P. J. Morrison, New Journal of Physics 19, 015007 (2017).

[147] E. Tassi, P. L. Sulem, and T. Passot, J. Plasma Phys. 82, 705820601 (2016).

[148] K. H. Kiyani, S. C. Chapman, F. Sahraoui, B. Hnat, O. Fauvarque, and Y. V. Khotyaintsev, Astrophys. J. 663, 10 (2013).

[149] G. Manfredi, Fields Institute Communications Series, 46, 263 (2005).

[150] M. Bonitz, AIP Conf. Proc. 1421, 135 (2012).

[151] S. A. Khan and M. Bonitz, "Quantum hydrodynamics," in Complex Plasmas: Scientific Challenges and Technological Opportunities, edited by M. Bonitz, K. Becker, J. Lopez, and 
H. Thomsen (Springer, Berlin, Heidelberg, 2014).

[152] M. Lingam, Comm. Nonlin. Sci. and Numer. Simul. 28, 022105 (2015).

[153] P. J. Morrison, Accepted for publication in Phys. Plasmas. Available at https://arxiv.org/pdf/1612.06734.pdf (2017).

[154] O. Ohia, J. Egedal, V. S. Lukin, W. Daughton, and A. Le, Phys. Rev. Lett. 109, 115004 (2012). 